\title{
Angular structure of jet quenching within a hybrid strong/weak coupling model
}

\author{
Jorge Casalderrey-Solana, ${ }^{a, b}$ Doga Can Gulhan, ${ }^{c}$ José Guilherme Milhano, ${ }^{d, e, f}$ \\ Daniel Pablos ${ }^{b}$ and Krishna Rajagopal ${ }^{g}$ \\ ${ }^{a}$ Rudolf Peierls Centre for Theoretical Physics, University of Oxford, \\ 1 Keble Road, Oxford OX1 3NP, United Kingdom \\ ${ }^{b}$ Departament de Física Quàntica i Astrofísica 8 Institut de Ciències del Cosmos (ICC), \\ Universitat de Barcelona, Martí $i$ Franquès 1, 08028 Barcelona, Spain \\ ${ }^{c}$ CERN, EP Department, \\ CH-1211 Geneva 23, Switzerland \\ ${ }^{d}$ CENTRA, Instituto Superior Técnico, Universidade de Lisboa, \\ Av. Rovisco Pais, P-1049-001 Lisboa, Portugal \\ ${ }^{e}$ Laboratório de Instrumentação e Física Experimental de Partículas (LIP), \\ Av. Elias Garcia 14-1, P-1000-149 Lisboa, Portugal \\ ${ }^{f}$ Theoretical Physics Department, CERN, \\ Geneva, Switzerland \\ ${ }^{g}$ Center for Theoretical Physics, Massachusetts Institute of Technology, \\ Cambridge, MA 02139 U.S.A. \\ E-mail: jorge.casalderreysolana@physics.ox.ac.uk, dgulhan@mit.edu, \\ guilherme.milhano@tecnico.ulisboa.pt, dpablos@fqa.ub.edu, \\ krishna@mit.edu
}

ABSTRACT: Within the context of a hybrid strong/weak coupling model of jet quenching, we study the modification of the angular distribution of the energy within jets in heavy ion collisions, as partons within jet showers lose energy and get kicked as they traverse the strongly coupled plasma produced in the collision. To describe the dynamics transverse to the jet axis, we add the effects of transverse momentum broadening into our hybrid construction, introducing a parameter $K \equiv \hat{q} / T^{3}$ that governs its magnitude. We show that, because of the quenching of the energy of partons within a jet, even when $K \neq 0$ the jets that survive with some specified energy in the final state are narrower than jets with that energy in proton-proton collisions. For this reason, many standard observables are rather insensitive to $K$. We propose a new differential jet shape ratio observable in which the effects of transverse momentum broadening are apparent. We also analyze the response of the medium to the passage of the jet through it, noting that the momentum 
lost by the jet appears as the momentum of a wake in the medium. After freezeout this wake becomes soft particles with a broad angular distribution but with net momentum in the jet direction, meaning that the wake contributes to what is reconstructed as a jet. This effect must therefore be included in any description of the angular structure of the soft component of a jet. We show that the particles coming from the response of the medium to the momentum and energy deposited in it leads to a correlation between the momentum of soft particles well separated from the jet in angle with the direction of the jet momentum, and find qualitative but not quantitative agreement with experimental data on observables designed to extract such a correlation. More generally, by confronting the results that we obtain upon introducing transverse momentum broadening and the response of the medium to the jet with available jet data, we highlight the importance of these processes for understanding the internal, soft, angular structure of high energy jets.

KeYwords: Heavy Ion Phenomenology, Jets

ARXIV EPRINT: 1609.05842 


\section{Contents}

1 Introduction 1

2 Brief summary of the hybrid model 5

3 Transverse kicks and jet broadening $\quad 7$

3.1 Introduction of broadening into the hybrid model $\quad 9$

$\begin{array}{lll}3.2 & \text { Insensitivity of jet observables to broadening } & 10\end{array}$

$\begin{array}{lll}3.3 & \text { An observable that is sensitive to broadening } & 17\end{array}$

4 Medium backreaction: observable consequences of a wake in the plasma 21

4.1 The spectrum of hadrons from a medium perturbed by the passage of a jet 24

4.2 Implementation of backreaction, background model, and jet hadronization 26

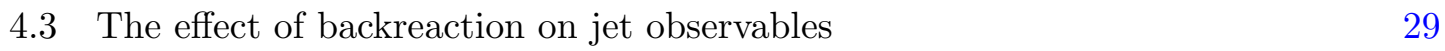

4.4 Recovering the "lost" jet energy and "missing" $p_{\mathrm{T}}$ deposited by a dijet pair 37

5 Discussion and outlook $\quad 46$

$\begin{array}{ll}\text { A Transverse kicks kinematics } & 51\end{array}$

B Background subtraction procedure $\quad 52$

\section{Introduction}

High energy heavy ion collisions provide a unique opportunity to explore the properties of hot, deconfined, strongly interacting matter, called quark-gluon plasma (QGP). The study of these collisions at the Relativistic Heavy Ion Collider (RHIC) and at the Large Hadron Collider (LHC) has demonstrated that matter at temperatures above the crossover between hot hadronic matter and hotter QGP exhibits strong collective phenomena [1-7] which can be described successfully by hydrodynamic simulations of the rapid expansion and cooling of the initially lumpy droplets of matter produced in the collisions [8-18]. Such strong collectivity has also recently been observed in smaller colliding systems, including $\mathrm{p}-\mathrm{Pb}$, p-p or ${ }^{3} \mathrm{He}-\mathrm{Au}$ [19-29], for which hydrodynamic simulations also seem to be successful [3037]. The applicability of hydrodynamics from early times in the evolution and for small systems suggests that the matter formed in these ultrarelativistic collisions is a strongly coupled liquid. Support for this picture comes from analyses of collisions in strongly coupled gauge theories with a dual holographic description which show that collisions of objects with transverse size $R$ produce a droplet of strongly coupled liquid that can be described hydrodynamically as long as the collisions are energetic enough that the temperature of the liquid at the time that it hydrodynamizes, $T_{\text {hydro }}$, satisfies $R T_{\text {hydro }} \gtrsim 1[38,39]$ and that in 
these collisions and in the collisions of objects that are infinite in transverse extent [40-42] hydrodynamization occurs at a time of order $1 / T_{\text {hydro }}$ after the collision.

The discovery that the QGP that filled the microseconds-old universe and that is recreated in collisions at RHIC and the LHC is a strongly coupled liquid challenges us to understand how such a liquid emerges from an asymptotically free gauge theory. When probed at very short length scales, the strongly coupled QGP of QCD (unlike the strongly coupled plasmas in theories with holographic descriptions) must consist of weakly coupled quarks and gluons. This makes constraining the microscopic nature of QGP via studying its interaction with energetic probes an important and interesting long-term goal. Some of the most salient examples of such probes are QCD jets. As a partonic jet shower propagates through the strongly coupled plasma created in a heavy ion collision, it suffers a strong process of energy loss as a result of its interactions with the plasma. The partons in the jet also pick up momentum transverse to their direction of motion as they are jostled during their passage through the medium. These interactions lead to a reduction in the jet energy (or quenching) and to modifications of the properties of jets produced in heavy ion collisions relative to those of their counterparts produced in proton-proton collisions, that propagate in vacuum. These interactions also result in the transfer of energy and momentum to the plasma: the jets create a wake as they lose energy. By pursuing a large suite of jet measurements, the different LHC collaborations have observed strong modification of different jet observables in heavy ion collisions [43-61], making jets promising probes for medium diagnostics. The first experimental constraints on jet quenching came from hadronic measurements at RHIC [62-64]. Analyses of jets themselves and their modification are also being performed at RHIC [65-68] and are one of the principal scientific goals of the planned sPHENIX detector [69].

To fully exploit their potential as tomographic probes, a detailed understanding of the interactions of jet showers with hot QCD matter is needed. A complete theoretical description of these processes is a challenging task, due to the multi-scale nature of jet probes. On the one hand, the production of jets and the processes via which an initial hard parton fragments into a shower are governed by short distance physics that is weakly coupled. On the other hand, the interaction of jets with the medium, as well as the dynamics of softer components within jets, are sensitive to the strongly coupled dynamics of the plasma at scales of order its temperature.

One class of approaches toward making progress on this difficult theoretical problem that has been pursued intensively starts from the premise that the dynamics of the plasma itself are weakly coupled, as if the temperature of the plasma were asymptotically large, and from the premise that the interactions of energetic partons and jets with the plasma are also governed entirely by weakly coupled physics. (See refs. [70-75] for reviews.) Based on these approaches, Monte Carlo tools for analyzing jet observables are being developed [7683] and many phenomenological studies of jets in medium have been confronted with LHC measurements of a variety of jet observables [81-109], including intrajet observables like those that we shall focus on $[85,89,99,101,104,106-109]$.

However, the observation that QGP is a strongly coupled liquid tells us that physics at scales of order its temperature is governed by strong coupling dynamics. This realiza- 
tion has lead to many fruitful connections between the physics of the QCD plasma and the gauge/gravity duality [110]. This technique allows us rigorous and quantitative access to nonperturbative, strongly coupled, physics - including thermodynamics, transport coefficients, hydrodynamics, thermalization, response to hard probes and other real time dynamics far from equilibrium - in a large family of non-abelian gauge theory plasmas that have a dual holographic description in terms of a black hole spacetime in a gravitational theory with one higher dimension. Although the current formulation of the duality has not been shown to apply to QCD, the study of the plasmas in gauge theories that do have a holographic description has led to many insights into the dynamics of hot deconfined matter in QCD. (See refs. [111-113] for reviews.) Within this context, there have been many interesting studies that address varied aspects of the interaction between high energy probes and strongly coupled plasma [114-147]. However, in all the examples that are currently directly accessible via gauge/gravity duality, the gauge theory remains strongly coupled in the ultraviolet, which limits the direct applicability of these results for phenomenological applications.

To address the multifaceted dynamics of QCD jets in strongly coupled plasma more fully, in refs. [148, 149] we introduced a phenomenological hybrid strong/weak coupling approach to analyzing jet quenching. In this approach, we treat different physics processes of relevance for the interaction of developing jet showers with the quark gluon plasma differently. In our model, the production and evolution of the jet shower is treated perturbatively, because the physics governing these processes is expected to be weakly coupled. And, we model the interaction between each of the partons formed in the shower with the medium using the result for the rate of energy loss of an energetic quark in strongly coupled plasma obtained via holographic calculations in refs. [143, 145]. The Monte Carlo implementation of this hybrid model has been successful in describing LHC measurements of a large suite of single jet, dijet and photon+jet observables [148, 149] and has been used to make predictions for more such observables and for $\mathrm{Z}+$ jet observables [149]. To date, the model has contained only a single free parameter, with all its successes and predictions having been obtained after fitting this parameter to a single measured data point.

In this paper, after reviewing the construction of our hybrid model briefly in section 2 we will extend the model in order to be able to use it to address the angular distribution of the energy within a jet and its modification via its passage through the plasma, as well as the angular distribution of the energy lost by jets during their passage through the plasma. To do so, we will supplement our model with two important physics processes which were absent in our previous implementations. First, in section 3 we add "transverse momentum broadening", namely the deflection to the direction of propagation of partons as a result of the exchange of momentum transverse to their direction of motion between the parton and the medium. We assume a Gaussian distribution of the magnitude of the momentum transfer and introduce one further model parameter to specify the width of the Gaussian. Second, in section 4 we add a simplified model for the collective response of the medium to the passage of the jet, namely the wake in the plasma that carries the energy and momentum lost by the jet and deposited in the plasma. We shall frequently refer to this as the "backreaction of the medium". We shall not actually focus on the form of the wake itself, 
focusing instead on the soft hadrons in the final state that result from the hadronization of the plasma including the wake and that carry the net momentum and energy lost by the jet. We make simplifying assumptions that allow us to add a representation of the effects of the wake on the final state hadrons that respects energy and momentum conservation, without introducing any new parameters into our hybrid model. Our principal assumption is that the energy lost by the jet thermalizes quickly, in the sense that it quickly becomes a hydrodynamic wake in the plasma that carries the "lost" energy and momentum, which in turn after freezeout becomes soft particles spread over a calculable and wide range of angles relative to the jet. This is an immediate and natural consequence of strong coupling dynamics [123]. Something similar can happen at weak coupling even though the energy is initially lost by gluon radiation because these radiated gluons can experience a cascade of reinteractions that converts the energy into soft particles at large angles [150-156]. The effects of both transverse momentum broadening [85, 86, 107-109] and the backreaction of the medium [95, 157-162] on jet observables have also been studied within the context of perturbative energy loss mechanisms.

After adding broadening and backreaction to our hybrid model, over the course of sections 3 and 4 we will assess their effects on measurements of different jet and dijet observables that are sensitive to the angular structure of jets, including the dependence of jet suppression on the reconstruction parameter $R$ which controls the angular size of the jets that are found and reconstructed in a sample of events, several observables based upon the jet shape including a new differential jet shape ratio that we introduce, the dijet acoplanarity, and the balance of momenta along the dijet axis carried by particles in the event with a given momentum. We also return to the jet fragmentation function, as we wish to see how it is modified by the soft particles coming from the backreaction of the medium.

One of our significant findings is that jets with a given energy that were produced in a heavy ion collision and so have had to traverse a droplet of QGP have a narrower hard jet core than jets with the same energy that were produced in vacuum, even if a substantial degree of transverse momentum broadening is turned on. This is a consequence of the fact that wider jets typically contain more, and less hard, partonic fragments and lose more energy than narrower jets with the same energy. The same phenomenon has been observed in calculations of jet quenching that are entirely done at strong coupling $[145,147]$ and in Monte Carlo calculations of radiative energy loss that are entirely done at weak coupling [105]. We find that this observation leads to two unexpected consequences. First, jets with a larger reconstruction parameter $R$ are more suppressed (the suppression factor $R_{\mathrm{AA}}$ of jets is pushed downward) at least for moderate $R$. Second, the intrajet angular distribution of energy in the quenched jets is remarkably independent of the amount of transverse momentum broadening. Note that we are only able to see that energy loss causes jets with a given energy to be narrower in heavy ion collisions than in protonproton collisions because we have incorporated event-by-event (maybe better phrased jetby-jet) variations in the fragmentation pattern of jets; this physical effect, and its striking consequences, are absent in approaches based on analyzing how an average jet is modified by passage through the plasma, as for example in refs. [107, 109]. 
Although we find that both the standard jet shape and the dijet acoplanarity are remarkably independent of the amount of transverse momentum broadening, we are able to construct a new observable - essentially a jet shape ratio that is partially differential in $p_{\mathrm{T}}$ - that is sensitive to the amount of transverse momentum broadening that we introduce. This points out a path toward detecting experimental evidence for the effects of transverse momentum broadening within a jet, which would be a very important first step toward using jets to resolve the microscopic structure of quark-gluon plasma.

Our last significant finding is that when we implement the collective response of the medium to the passage of the jet, the energy lost from the jet ends up in the form of soft particles separated from the jet axis by very large angles and is in qualitative agreement with experimental measurements of observables that are referred to as "missing- $p_{\mathrm{T}}$ " distributions which have recently been reported by CMS [60]. Careful comparison between our calculations of these and several other observables and experimental data in section 4 indicates that our simplified treatment of the wake produces slightly too many very soft particles $\left(p_{\mathrm{T}}<2 \mathrm{GeV}\right)$ at large angles and not enough particles with momenta in the $2-4 \mathrm{GeV}$ range. This is not entirely unexpected because the approximations via which we treat the particles coming from the wake are reliable only for particles with momenta that are not a lot larger than the freezeout temperature, but it may also be an indication that the wake in the plasma does not actually thermalize as fully as we assume in our simplified treatment.

We close in section 5 by discussing the various results of our analyses, identifying further improvements of our implementation of the in-medium dynamics of jets in strongly coupled plasma for the future, and looking ahead at the path toward using jets to resolve the microscopic properties of quark-gluon plasma.

\section{Brief summary of the hybrid model}

In this section, we provide a brief description of the hybrid model which we will employ to describe the modification of jets produced in heavy ion collisions that propagate through a droplet of hot matter relative to those produced in proton-proton collisions that propagate in vacuum. A more detailed account of the model may be found in refs. [148, 149].

The main motivation for introducing this model is to separate the strongly coupled dynamics of quark-gluon plasma itself and of interactions between it and partons plowing through it from the weakly coupled dynamics governing the production, showering and relaxation of virtuality of high energy QCD jets. Since for any parton that showers and forms a jet the initial virtuality of the parton is much larger than any scale associated with the medium, of order its temperature $T$, the first assumption of the model is that the evolution of the jet proceeds as in vacuum, with the branching of the parton shower unmodified by the presence of the strongly interacting plasma. Upon making this assumption, the modification of jet showers is only due to the interaction of each of the partons in the jet with the strongly coupled medium. After associating each parton in the jet with a life-time determined via a formation time argument $[148,149,163]$, we compute the energy lost by each parton as it propagates in the strongly coupled plasma. Since the interactions of each 
of these partons with the medium is sensitive to the medium scale, the rate of energy loss is controlled by strongly coupled dynamics. The second assumption of the model is that the rate at which a parton loses energy can be modeled by the rate of energy loss of light quark jets in the strongly coupled plasma of $\mathcal{N}=4$ supersymmetric Yang-Mills (SYM) theory which has been computed via holography and is given by [143, 145]

$$
\left.\frac{d E}{d x}\right|_{\text {strongly coupled }}=-\frac{4}{\pi} E_{\text {in }} \frac{x^{2}}{x_{\text {stop }}^{2}} \frac{1}{\sqrt{x_{\text {stop }}^{2}-x^{2}}},
$$

with $x_{\text {stop }}$ the distance over which the light quark jet would lose all of its energy if it propagated through plasma at a constant temperature $T$. In $\mathcal{N}=4 \mathrm{SYM}$ theory, jets with a given initial energy $E_{\text {in }}$ can have a wide range of initial opening angles, with the narrower jets having larger values of $x_{\text {stop }}$ [145]. There is a minimum possible initial opening angle, corresponding to the maximum possible $x_{\text {stop }}$ for jets with a given initial energy [145] that was computed holographically in refs. [131, 137, 141] and is given by

$$
x_{\text {stop }}=\frac{1}{2 \kappa_{\mathrm{sc}}} \frac{E_{\mathrm{in}}^{1 / 3}}{T^{4 / 3}},
$$

with $\kappa_{\mathrm{sc}}=1.05 \lambda^{1 / 6}$. In the hybrid model, we apply (2.1) parton-by-parton to each parton in a QCD DGLAP shower as described by PYTHIA [164], rather than attempting to use the $\mathcal{N}=4 \mathrm{SYM}$ jets that lose energy at the rate (2.1) themselves as models for QCD jets in plasma, as in ref. [147]. Because we apply (2.1) to individual partons, we use the form (2.2) for $x_{\text {stop }}$ appropriate for the skinniest possible $\mathcal{N}=4 \mathrm{SYM}$ jets. We shall further assume that the most salient differences between the strongly coupled limit of $\mathcal{N}=4$ SYM theory and QCD can be incorporated via varying the value of $\kappa_{\mathrm{sc}}$, which becomes the only fitting parameter of the hybrid model formulated in refs. [148, 149].

We have implemented this hybrid model into a Monte Carlo simulation in which hard jets, showering as described by PYTHIA [164], are embedded within a droplet of hot matter produced in a heavy ion collision, expanding and cooling as described by relativistic viscous hydrodynamics. To generate the hard QCD jet shower, we employ PYTHIA 8.183 [164], allowing the DGLAP shower to evolve down to a minimum transverse momentum of $p_{\mathrm{T}}{ }^{\mathrm{min}}=1 \mathrm{GeV}$. We distribute these hard events in the transverse plane of the heavy ion collisions according the number of binary nucleon-nucleon collisions. The trajectory of each jet is tracked from the generation point as it propagates through the expanding cooling plasma until the jet reaches a region where the temperature has dropped below a temperature $T_{c}$, below which we assume no further energy loss occurs. This $T_{c}$ is not sharply defined but it should presumably be near the crossover between quark-gluon plasma and hadronic matter and we therefore vary its value over the range $145<T_{c}<170 \mathrm{MeV}$. Seeing how our results vary as we vary $T_{c}$ over this range serves as a gauge of some of the uncertainties in our model. The energy loss rate is computed via eq. (2.1), with $x_{\text {stop }}$ evaluated according to eq. (2.2) at the local temperature as given by the hydrodynamic simulations of refs. $[16,17]$. Flow effects are taken into account by evaluating the rate of energy loss in the local fluid rest frame, as explained in ref. [149]. In refs. [148, 149], 
we have fitted the value of the parameter $\kappa_{\mathrm{sc}}$ to a single measurement, the suppression of the number of jets with one transverse momentum in LHC heavy ion collisions with one centrality, and have then successfully confronted this hybrid model with measurements of many single jet, dijet and $\gamma$-jet observables as functions of jet transverse momentum and collision centrality and made predictions for many further measurements of these types.

In the next sections, we shall extend our implementation of the hybrid model to include two new physics processes, transverse momentum broadening in section 3 and the response of the medium to the jet in section 4 , and will evaluate their consequences for intra-jet observables, in particular those related to the angular structure of jets.

\section{$3 \quad$ Transverse kicks and jet broadening}

Previously in refs. [148, 149], all the effects of the strongly coupled medium on the properties of the jets arise as a consequence of the energy lost by the partons in the jet shower as they plow through the medium. In this section, we augment our hybrid model by adding a second physical process, and hence a second free parameter, namely the kicks transverse to their direction of motion that the partons in the jet receive as a consequence of plowing through the medium. This process has long been referred to as "transverse momentum broadening" based upon the expectation that the consequence of the kicks in random transverse directions received by the many partons in a jet shower will be broadening of the jet. In perturbative calculations, transverse momentum broadening arises from the multiple soft exchanges of momentum that a parton suffers as it traverses a medium leading to a random change in its momentum and in particular providing the parton with some additional momentum perpendicular to its original direction of propagation.

If the energetic parton suffers multiple soft exchanges as it traverses the medium, the distribution of the momentum transferred via this stochastic process is well approximated as Gaussian. As a consequence, the transverse momentum distribution of partons that have traversed a medium of length $L$ is approximately Gaussian with a width that scales with the medium length, $Q_{\perp}^{2}=\hat{q} L$. The quantity $\hat{q}$ that arises here is called the momentum broadening parameter; this property of the medium codifies the typical squared momentum that the medium transfers to the probe per unit length. It has dimension 3 and in a plasma in thermal equilibrium with temperature $T$ it is proportional to $T^{3}$, up to a possible logarithmic dependence on the ratio of the parton energy to $T$. In perturbative calculations of energy loss via gluon radiation, the medium parameter $\hat{q}$ also determines the intensity of the gluon radiation induced by the medium, and hence is related to energy loss as well as to momentum broadening. In our model, we introduce $\hat{q}$ only as a way of parametrizing momentum broadening.

In the strongly coupled plasma of $\mathcal{N}=4$ SYM theory, transverse momentum broadening has been calculated holographically for both heavy quarks $[116,121,122]$ and massless quarks $[115,132]$. Although there is no notion of scattering centers and no notion of multiple discrete transfers of momentum in the strongly coupled limit, both heavy and massless quarks pick up transverse momentum as they propagate through the hot strongly coupled liquid and the resulting transverse momentum distribution is Gaussian with a width 
$Q_{\perp}^{2}=\hat{q} L$ with $\hat{q} \propto \sqrt{\lambda} T^{3}$, with $\lambda$ the 't Hooft coupling. However, unlike at weak coupling there is no strong correlation between the dynamics responsible for transverse momentum broadening and that responsible for parton energy loss.

We shall introduce broadening into our hybrid model by assuming a Gaussian distribution for the transverse momentum picked up by each parton in the shower, with the momentum squared picked up per distance travelled given by

$$
\hat{q}=K T^{3}
$$

with $T$ the local temperature of the medium at the location of a given parton at a given time and with $K$ a theory-dependent constant that we shall treat as a free parameter that should ultimately be determined via fitting to data. Since the medium is dynamical, with longitudinal and transverse expansion, $T$ and hence $\hat{q}$ varies with position and time. We shall obtain this dependence from the hydrodynamic simulation of the expanding cooling droplet of plasma in our hybrid model.

For massless or very energetic particles, in $\mathcal{N}=4$ SYM theory in the large number of colors $\left(N_{c}\right)$ limit the value of $K$ has been calculated holographically [115, 132] and is given by $K=K_{\mathcal{N}=4} \simeq 24$ for $\lambda=10$, a value of the 't Hooft coupling $\lambda=g^{2} N_{c}$ that corresponds to $g^{2} / 4 \pi \simeq 0.27$ for $N_{c}=3 . K_{\mathcal{N}=4}$ is proportional to $\sqrt{\lambda}$ as noted above. In a large class of conformal theories $K_{\mathrm{CFT}} / K_{\mathcal{N}=4}=\sqrt{s_{\mathrm{CFT}} / s_{\mathcal{N}=4}}[120], s$ being the entropy density. This suggests that $K_{\mathrm{QCD}}$ is likely to be less than $K_{\mathcal{N}=4}$ since, at least at weak coupling, $s_{\mathrm{QCD}} / s_{\mathcal{N}=4} \simeq 0.40$. An alternative approach to gaining an expectation for the likely value of $K_{\mathrm{QCD}}$ is to start from a perturbative analysis of parton energy loss, in which the value of $K$ controls energy loss via gluon radiation and, via this relation, can be related to experimental observables that are sensitive to parton energy loss like for example the suppression in the number of high-momentum hadrons in heavy ion collisions as compared to proton-proton collisions. The JET Collaboration has pursued this approach [165]; the value of $\hat{q}$ that they have found corresponds to a value of $K$ given by $K_{\text {pert }} \simeq 5$. To date, nobody has extracted a value of $K$ via comparison to data on experimental observables that are directly sensitive to transverse momentum kicks and jet broadening. (A recent pioneering attempt [109] yields values ranging from 0 to several times larger than the value of $K_{\text {pert }}$ obtained in ref. [165].) Our goal in this section is to introduce transverse momentum broadening into our hybrid model, treating $K$ as a parameter that should ultimately be determined via comparison between calculations of the ( $K$-dependent) observable consequences of momentum broadening to experimental measurements of observables that are directly sensitive to this physical process.

In this section, we will use our hybrid model to analyze the consequences of transverse momentum broadening for various different jet observables. Based upon the discussion above, we expect that $K$ actually lies somewhere around 5 to 20. But, in order to better understand the consequences of broadening, we shall investigate the effects on observables of varying $K$ over the wide range $0 \leq K \leq 100$. Our principal conclusion, after investigating a suite of jet observables, will be that most observables, even those tailored to measuring the angular structure of jets, are remarkably insensitive to broadening, showing little sensitivity to $K$ over the full range that we explore. This conclusion becomes less surprising once we 
recall that even if every individual jet is broadened by its passage through the medium, jets with a given energy can end up being narrower in heavy ion collisions than in proton-proton collisions. This happens if wider jets lose more energy than narrower jets (as is the case in perturbative calculations in QCD [166] and in holographic calculations of jets in strongly coupled $\mathcal{N}=4 \mathrm{SYM}$ theory [145]) and if the probability distribution for the jet production absent any medium effects is a steeply falling function of jet energy, as is the case. Recent weakly coupled Monte Carlo calculations of an ensemble of QCD jets in heavy ion collisions as compared with those in proton-proton collisions [105] and recent holographic analyses of how propagation through plasma modifies the energy and opening angle distribution of an ensemble of $\mathcal{N}=4$ SYM jets with initial distributions as in perturbative QCD [147] both provide clear illustrations of how jets with a given energy can end up narrower in heavy ion collisions even though every individual jet broadens as it propagates through plasma. We shall find the same in our hybrid model. The resulting insensitivity of jet observables to the value of $K$ will make it quite challenging to extract the value of this medium parameter from data.

In section 3.3 we shall introduce a new observable which does exhibit considerable sensitivity to in-medium transverse momentum broadening, proposing this observable as a possible route to using future experimental measurements to constrain the value of $K$.

\subsection{Introduction of broadening into the hybrid model}

We shall assume that each parton in a jet shower picks up some transverse momentum as it propagates through the plasma for a time $d t$, with the transverse direction chosen randomly and with the magnitude of the momentum chosen from a Gaussian distribution with a width $\hat{q} d t$ with $\hat{q}$ specified in terms of the local temperature of the plasma $T$ and the parameter $K$ according to (3.1). Many previous computations of in-medium energy loss and broadening of jets have been performed in the limit of a static fluid at rest. However, we shall study the interactions of jets with an expanding, cooling, droplet of plasma as described by hydrodynamics. As in our previous analysis of jet observables related to parton energy loss [149], we shall apply results appropriate to a static medium in the local fluid rest frame, meaning in the present case that we are neglecting any effects of gradients in the fluid on transverse momentum broadening. This prescription implies that all modifications to the momentum of the parton (loss of longitudinal momentum according to (2.1) as previously and the transverse momentum kick that we are introducing here) are computed in the rest frame of the fluid at the location of the parton at a given time. Note that the transverse momentum kicks are transverse to the direction of motion of the parton in the local fluid rest frame, meaning that they need not be transverse to its momentum in the collision center-of-mass frame. We relegate the details of the transformation between the fluid frame and the collision frame to appendix A.

Following our Monte Carlo approach, we perform a full simulation of an ensemble of jets. For the analysis performed in this section, we studied 500,000 jet events, generated and evolved by PүтніA 8.183 [164]. The point of origin in the transverse plane of the hard processes is distributed by sampling the binary collison probability density while initial transverse direction and rapidity are retained from PYTHIA. 
We embed these jets in the hydrodynamic background of refs. [16, 17]. We follow the trajectories of all the partons in the PYTHIA jet shower as they traverse the hot matter created in the collision, as described in the hydrodynamic simulation. Between the time when each parton is created at a branching event and the time when each parton itself branches, we discretize its trajectory and at each point we add to its momentum a random transverse momentum chosen according to a Gaussian distribution of width $\hat{q} d t_{F}$ with $d t_{F}$ the length of the discretized time interval in the fluid frame. We have checked that our results do not depend on our choice of $d t_{F}$.

As described in detail in appendix A, we implement broadening in the local fluid rest frame by assuming that in this frame the energy and virtuality of a parton do not change when the parton is kicked; the only thing that changes due to the tranverse kicks it receives is the direction of the parton's momentum vector. Again as described in detail in appendix A, upon boosting back from the local fluid rest frame to the collision center of mass frame there will in general be a change to the collision frame energy of the parton. It turns out that more often than not in the collision frame the partons lose a small amount of energy as a consequence of the transverse kicks they receive. In addition, the transverse kicks they receive may push some partons outside the jet. (Since the jet shape falls with distance from the jet axis, although transverse kicks may also push partons into the jet this is less likely.) These effects together mean that, summed over the whole jet evolution, the transverse momentum kicks that we are adding will result in a slight increase to the overall jet energy loss at a fixed value of $\kappa_{\mathrm{sc}}$. That is, the dynamics of broadening leads to a small increase in the quenching of jets. This means that for each nonzero value of $K$ we need to refit the value of the parameter $\kappa_{\mathrm{sc}}$ that, through (2.1) and (2.2), determines the amount of energy loss in our hybrid model. We do so in figure 1, finding that the effect is small. For values of $K$ around 5 to 20, the effect of broadening implies a reduction of less than $5 \%$ in the value of $\kappa_{\mathrm{sc}}$ relative to that reported in our previous work. This effect is much smaller than the uncertainties represented by the width of the band in figure 1 . Even for the extreme value $K=100, \kappa_{\mathrm{sc}}$ is only reduced by about $10 \%$.

\subsection{Insensitivity of jet observables to broadening}

Having fixed the dependence of the quenching parameter $\kappa_{\mathrm{sc}}$ on the broadening parameter $K$, we can now begin our exploration of the effect of broadening on different observables. With an ensemble of events in hand, generated by PYтнIA and modified to include energy loss as described in section 2 and broadening as described above, the first step in the calculation of any jet observable is the finding and reconstruction of jets in each of the events in the ensemble. We do so using the anti- $k_{t}$ algorithm [167] as implemented in the FastJet package [168].

The first observable that we consider is the suppression factor of jets (namely the ratio $R_{\mathrm{AA}}^{\text {jet }}$ of the number of jets with specified kinematics in heavy ion collisions to the number of jets with the same kinematics in proton-proton collisions) as a function of the jet reconstruction parameter $R$ that arises in the anti- $k_{t}$ algorithm [167]. The anti- $k_{t}$ algorithm groups particles within $R$ of each other in the $(\eta, \phi)$ plane into what it defines as a single jet whereas if $R$ were smaller it may reconstruct the same particles as several smaller 


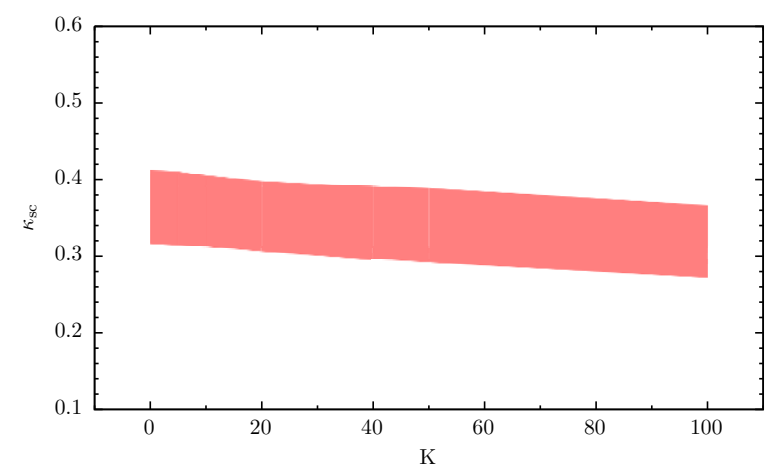

Figure 1. When we introduce transverse momentum broadening via a nonzero value of the broadening parameter $K$, this introduces a small increase in energy loss. This means that for each nonzero value of $K$ we need to refit the value of the energy loss parameter $\kappa_{\text {sc }}$ to the measured value [49] of $R_{\mathrm{AA}}^{\text {jet }}$ for jets with $100 \mathrm{GeV}<p_{\mathrm{T}}<110 \mathrm{GeV}$ and $-2<\eta<2$, as in refs. [148, 149]. The resulting dependence of $\kappa_{\mathrm{sc}}$ on the broadening parameter $K$ is mild. As in refs. [148, 149], the width of the band of values of $\kappa_{\mathrm{sc}}$ in this figure (and the consequent widths of the bands depicting our hybrid model predictions in subsequent figures) comes both from the error bar on the experimentally measured data point used to fix $\kappa_{\mathrm{sc}}$ and from varying the crossover temperature $T_{c}$ as described in section 2 in order to get some sense of the systematic uncertainties in the hybrid model.
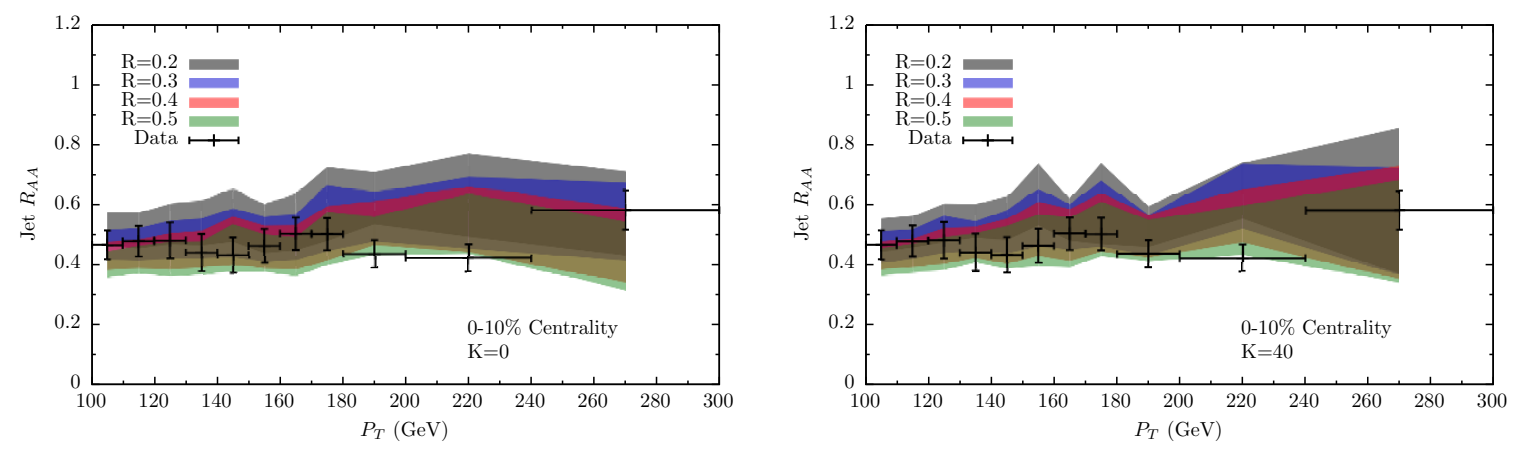

Figure 2. Dependence of $R_{\mathrm{AA}}^{\mathrm{jet}}$ on the anti- $k_{t}$ jet reconstruction parameter $R$ for $K=0$ (no broadening, left panel) and $K=40$ (right panel). For comparison, we show the suppression of jets reconstructed using the anti- $k_{t}$ algorithm with $R=0.3$ as measured by CMS in the same interval of rapidity [49].

narrower jets. Hence, choosing a larger $R$ translates into reconstructing an ensemble of jets that tend to be wider in angle. Because of this, the dependence of the suppression factor $R_{\mathrm{AA}}^{\mathrm{jet}}$ on $R$ is often considered a proxy for modification of the angular structure of jets as a consequence of their interaction with the medium. A naive expectation, then, would be that turning on transverse momentum broadening should make jets broader and that this in turn should leave some imprint in the $R$-dependence of $R_{\mathrm{AA}}^{\mathrm{jet}}$, increasing the suppression for smaller $R$. This is not at all what we observe.

In figure 2 we show the $R$ dependence of the suppression factor $R_{\mathrm{AA}}^{\text {jet }}$ for $K=0$, i.e. no broadening, and for $K=40$ for jets with $|\eta|<2$ as a function of $p_{T}$. The data points come from measurements of jets reconstructed with $R=0.3$; the colored bands 
are the results from our model for jets reconstructed with four different values of $R$. The left-most data point in each panel is the one that we have used to constrain the value of $\kappa_{\mathrm{sc}}$, as we described in section 3.1. The calculations shown in the two panels therefore have different values of $\kappa_{\mathrm{sc}}$, although the difference is small. In both panels, although the suppression factor shows only a very weak dependence on the reconstruction radius $R$, wide (larger $R$ ) jets tend to be somewhat more suppressed than narrow (smaller $R$ ) jets. This is a trend that we foreshadowed in the introduction to section 3. When comparing jets at the same energy, wide jets contain more fragments (in our hybrid model, simply more partons) than narrow jets. Since the larger the number of partons traversing the medium the larger the lost energy, wide jets are naturally more suppressed. The same qualitative dependence of the suppression factor on the jet size has recently been observed in holographic computations $[145,147]$. Measurements of the $R$-dependence of $R_{\mathrm{AA}}$ for these high energy jets by the CMS collaboration show very little sensitivity to the value of $R$ [49], as in our simulations, although the uncertainties in our calculations and in the measurements preclude a quantitative comparison at present. We shall return to the $R$-dependence of $R_{\mathrm{AA}}$ in section 4 .

In spite of the extreme transverse momentum broadening introduced by choosing $K=$ 40 , the $R$-dependence of the observable plotted in figure 2 is almost identical in the two panels. The origin of this lack of sensitivity to $K$ lies in the strong quenching of jets by the plasma, and in particular in the fact that, as we have already noted, wider jets are more strongly quenched than narrower jets. In particular, the softer partons within a wider jet that could serve to further broaden the jet as they are kicked in transverse momentum instead lose almost all of their energy. This means that the jet sample that ends up dominating the inclusive jet spectrum ratio $R_{\mathrm{AA}}^{\mathrm{jet}}$ is biased such that most jets in the sample contain only a few or even just one hard parton. For such jets, transverse momentum kicks, even with an extreme value of $K$, serve only to change the direction of the jet axis, not to broaden the jet.

We turn now to the second of the three observables that we shall analyze in this section, one that we will use to look for exactly the change in the direction of the jet axis due to transverse momentum broadening, namely due to the vector-summed effect of the transverse momentum kicks felt by each of the partons in the jet. If we consider a dijet pair, the change in the direction of propagation of the two jets in the pair will in general differ, since broadening is a stochastic process and also since the temperature as a function of time along the trajectory of each of the jets will in general be different. If all dijet pairs were produced back-to-back in azimuthal angle, with $\Delta \phi=\pi$, deviations from $\Delta \phi=\pi$ due to different broadening-induced kicks to the two jets in the pair could be used as a direct measurement of broadening. Reality is not this simple. The hard scattering processes that produce dijets often include radiative production of more than two partons, and in many events where two jets are reconstructed there may in fact have been a third or even fourth jet present also. This means that even in proton-proton collisions there is a nontrivial distribution of $\Delta \phi$, centered around $\pi$ but with considerable width. We shall start from this distribution, and then look at the effects on it due to propagation through the plasma originating from energy loss and broadening. 

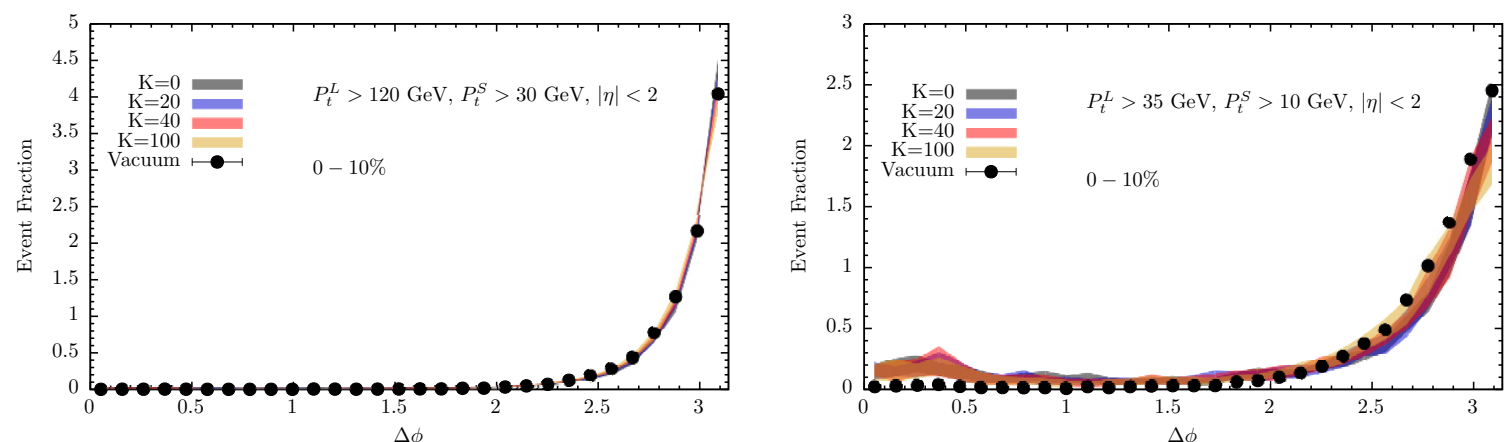

Figure 3. Dijet acoplanarity distribution for high-energy (left) and low-energy (right) dijets in LHC heavy ion collisions with $\sqrt{s}=2.76 \mathrm{ATeV}$ for two different values of the broadening parameter $K$. For comparison, the black dots show the acoplanarity in proton-proton collisions as simulated by PYTHIA.

Deviations of $\Delta \phi$ away from $\pi$ are termed acoplanarity because the two jet axes and the beam axis are not coplanar. The black dots in figure 3 shows the acoplanarity distribution for dijets in proton-proton collisions (propagating in vacuum) for two different cuts on the transverse momentum of the dijet pair: $p_{\mathrm{T}}^{\text {leading }}>120 \mathrm{GeV}$ and $p_{\mathrm{T}}{ }^{\text {subleading }}>30 \mathrm{GeV}$ (left) and $p_{\mathrm{T}}^{\text {leading }}>35 \mathrm{GeV}$ and $p_{\mathrm{T}}$ subleading $>10 \mathrm{GeV}$ (right) as simulated by PYTHIA. The $\Delta \phi$-distribution is different in the two momentum regions displayed, with the high-energy acoplanarity slightly narrower (closer to $\Delta \phi=\pi$ ) than the low energy one. This occurs because the fragmentation of higher energy jets leads to a narrower angular distribution of fragments than for lower energy jets, and in a case where only two jets are produced the acoplanarity will be less if the jets are narrower.

As already pointed out in refs. [89, 108, 109], in the case of the high energy dijet pairs the effects of broadening on their acoplanarity is much smaller than the width of the vacuum acoplanarity distribution. Indeed, in the left panel of figure 3 we see that our results with $K=0$ (no-broadening) and $K=100$ (extreme broadening) are both indistinguishable from the vacuum distribution.

In the right panel of figure 3, we look at the acoplanarity distribution for dijets with much lower energy, choosing dijets with $p_{\mathrm{T}}^{\text {leading }}>35 \mathrm{GeV}$ and $p_{\mathrm{T}}{ }^{\text {subleading }}>10 \mathrm{GeV}$. It is challenging, perhaps prohibitively so, to measure jets with energies as low as this in LHC heavy ion collisions, but even as an academic study the results are interesting. First, our $K=0$ calculation apparently yields a narrower $\Delta \phi$ distribution than in vacuum. One contribution to the origin of this apparent effect lies in the increase in the number of dijet pairs close to $\Delta \phi=0$; since what is plotted is a normalized probability distribution, this tends to lower the curve near $\Delta \phi=\pi$. The enhancement of almost collinear jet pairs is a consequence of energy loss and arises when a jet that had been propagating somewhere near $\Delta \phi=\pi$ loses so much energy that its energy drops below that of a third jet produced close to $\Delta \phi=0$, meaning that what is reconstructed is a dijet with $\Delta \phi \sim 0$. We have checked that if we restrict the dijet distribution to pairs of jets moving in opposite hemispheres, the $K=0$ and vacuum acoplanarity distributions are much more similar. The second reason 


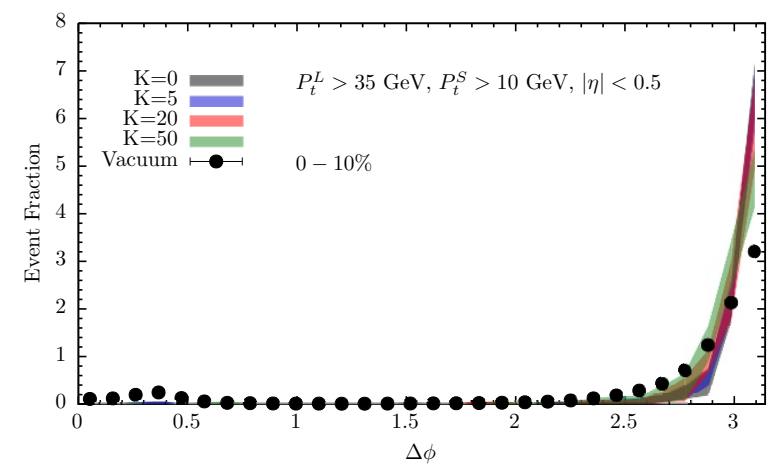

Figure 4. Dijet acoplanarity distribution for dijets in heavy ion collisions at RHIC for different values of the broadening parameter $K$. For comparison, the black dots show the acoplanarity distribution in proton-proton collisions as simulated by РҮтніA.

why the distribution of $\Delta \phi$ around $\pi$ is slightly narrower in heavy ion collisions than in vacuum is that a quenched jet seen in a heavy ion collision with a given energy began with a larger energy, and the acoplanarity distribution for higher energy dijets as produced in vacuum is narrower.

We can now look at the acoplanarity distribution with $K=100$ for the low energy dijets in the right panel of figure 3. We see that introducing this extreme degree of broadening does make the acoplanarity distribution very slightly wider than the $K=0$ distribution, perhaps by coincidence bringing it back into agreement with the vacuum acoplanarity distribution. The principal conclusion, though, is that in LHC heavy ion collisions, even for dijets with very low energies and even with substantially more broadening than is expected, the effects of broadening on the acoplanarity distribution are very small.

As noted in refs. [108, 109], the effects of broadening on acoplanarity distributions are larger for low energy jets at RHIC, where the smaller soft background makes these measurements more feasible. Furthermore, the vacuum acoplanarity distribution is much narrower in RHIC heavy ion collisions than at the LHC for dijets with a comparable range in transverse momentum. As we now explain, this can be attributed to the fact that the jet spectrum at a given jet energy is more steeply falling in lower energy RHIC collisions than it is at the LHC. One way in which dijets with a given energy pick up acoplanarity is if the subleading jet in the pair started out at a higher energy and radiated a relatively hard gluon, which became a third jet that balances the acoplanarity in the momenta of the reconstructed dijet pair. If at a given energy the spectrum is a more steeply falling function of energy this means that there are fewer initially higher energy jets that could radiate and end up at the given energy. Thus, a steeper spectrum as in RHIC collisions yields a narrower acoplanarity distribution. This is illustrated by the black dots in figure 4 that show the acoplanarity distribution in RHIC heavy ion collisions for dijet pairs with $p_{\mathrm{T}}^{\text {leading }}>35 \mathrm{GeV}$ and $p_{\mathrm{T}}{ }^{\text {subleading }}>10 \mathrm{GeV}$, the same dijet energies as in the right panel of figure 3 although here we have chosen dijets with $|\eta|<0.5$. Note that here the vacuum distribution shows a small accumulation of events at $\Delta \phi \lesssim 0.3$. This is a result of the narrow rapidity coverage $|\eta|<0.5$ : in a small fraction of events, the jet that balances 
most of the transverse momentum of the leading jet falls outside the accepted $\eta$ range and what is reconstructed as a dijet is the leading jet and a subleading jet pointing in a similar direction.

We see in figure 4 that the RHIC acoplanarity distribution becomes visibly narrower in heavy ion collisions if we neglect broadening, setting $K=0$. This effect is perhaps hinted at in the right panel of figure 3 but becomes clearly visible at RHIC (energy loss, and therefore this effect, are not included in the calculations of refs. $[108,109])$. It is a consequence of strong energy loss and a steeply falling jet spectrum in sum. Wider jets with a given energy loses more energy than narrower jets, meaning that the jets that start out at in a given energy bin and stay in that bin are the narrow jets. Because of the steeply falling spectrum, there are not many jets that originate with higher energies, lose energy, and end up in the given energy bin. The result is a narrowing of jets that remain in a given energy bin, something that has been seen previously in both perturbative [105] and holographic [145, 147] analyses. And, we see from the $K=0$ results in figure 4 that narrower dijets are less acoplanar. When we now turn on broadening, in addition to energy loss, the jets get broader and the acoplanarity increases. The effect of broadening is small for realistic values of $K$ in the 5-20 range; it takes unrealistically large values of $K$ to broaden the jets sufficiently that the acoplanarity distribution becomes as wide as in vacuum.

The lesson here is that acoplanarity is to some degree sensitive to both energy loss and broadening, more so for lower energy jets in lower energy collisions where the spectrum is more steeply falling. But, even in the best case, this observable exhibits little sensitivity to broadening, with the narrowing of the acoplanarity distribution due to energy loss being greater than the broadening of the acoplanarity distribution due to momentum broadening with realistic values of $K$. The competition between these two opposing effects would complicate any attempt to use acoplanarity measurements in isolation to see effects of broadening, leave apart to extract the value of the medium broadening parameter $K$.

The third observable that we shall analyze is called the jet shape and is an intrajet observable that is a measure of the angular distribution of the energy within a jet. The jet shape is defined as the fraction of the jet energy in jets reconstructed with a given anti- $k_{t}$ parameter $R$ that is contained within an annulus of radius $r$ and width $\delta r$ (in $\eta-\phi$ space) centered on the jet axis. Following the analysis in ref. [51], we define the differential jet shape as

$$
\rho(r) \equiv \frac{1}{N_{\text {jets }}} \frac{1}{\delta r} \sum_{\text {jets }} \frac{\sum_{i \in r \pm \delta r / 2} p_{t}^{i, \text { track }}}{p_{t}^{\text {jet }}}
$$

for $r<R$, where the tracks in the sum don't necessarily have to belong to the jet constituents defined through the anti- $k_{t}$ clustering. For this reason the final jet shape distribution is multiplied by the event averaged factor $\left\langle p_{t}^{\text {jet }} / \sum_{i=0}^{N_{\text {bins }}} p_{t}^{\text {track }}\left(r_{i}\right)\right\rangle$ so that it is normalized to one. We show the result of this analysis in figure 5, where we compare the ratio of the jet shape for the quenched jets in $\mathrm{PbPb}$ collisions to that for the unquenched jets in proton-proton collisions. In this analysis, the sum over jets in (3.2) includes all 

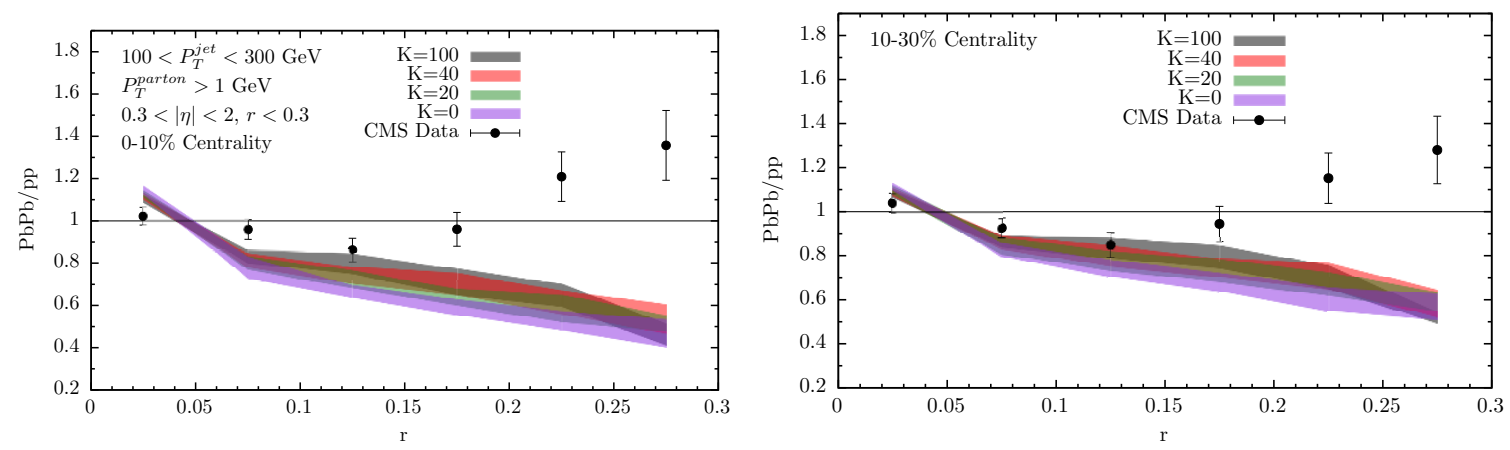

Figure 5. Left: ratio of the jet shape in $\mathrm{PbPb}$ collisions to that in proton-proton collisions for different values of the broadening parameter $K$ as compared to CMS data from heavy ion collisions with $\sqrt{s}=2.76 \mathrm{ATeV}[51]$ and $0-10 \%$ centrality (left) or 10-30\% centrality (right).

jets with $p_{t}^{\text {jet }}>100 \mathrm{GeV}$ and $0.3<|\eta|<2$. For reference, we also show the experimental results for this ratio, as measured by the CMS collaboration [51].

The hybrid model calculations with $K=0$ shown in figure 5 provide clear confirmation that, as we have discussed, energy loss serves to narrow the angular size of jets with a given energy in heavy ion collisions relative to that of jets with the same energy in protonproton collisions. Again, this arises because wider jets lose more energy than narrower jets, leaving behind a sample that is dominated by narrower jets. Adding transverse momentum broadening by turning on a nonzero value of $K$ serves to broaden the jets in the sample, slightly. The effect of broadening is very small even for the unrealistically large choice $K=100 .{ }^{1}$ (Note that transverse momentum picked up by the one or few hardest partons in the jet serves to deflect the angle of the reconstructed jet, affecting the acoplanarity. This has little effect on the jet shape since $r$ is measured relative to the center of the reconstructed jet, not relative to whatever the original direction of its parent parton was.) It is apparent in figure 5 that our analysis in this section does not do a good job of describing the jet shape ratio measured in experimental data [51], in particular at larger values of $r$. As we shall discuss in section 4, at larger and larger $r$ the partons in the reconstructed jet are softer and softer. Our hybrid model fails to describe a medium-induced enhancement in the production of soft particles at large angles relative to the jet direction seen in heavy ion collisions relative to proton-proton collisions. This enhancement, which does not contribute much to the overall jet energy, points to the existence of soft modes moving in the same direction as the jet, which is to say a moving wake in the plasma that the jet itself excites as it loses energy and momentum to the plasma. In our implementation of the hybrid

\footnotetext{
${ }^{1}$ In the results of ref. [107], introducing broadening results in larger effects on the jet shapes. There are at least two reasons for this. First, the analysis of ref. [107] focuses on the modification of average jets. Leaving out jet-by-jet fluctuations means that this analysis cannot see that wider jets lose more energy than narrower ones, and so does not include the consequent narrowing of jets with a given energy in $\mathrm{PbPb}$ collisions relative to jets with the same energy in proton-proton collisions. And, second, in ref. [107] all the partons present in a PYTHIA shower just before hadronization are assumed to have passed through the medium, and felt the effects of broadening, whereas in our calculation of the development of the jet shower in spacetime we see that many partons are produced at splittings that occur after the jet has already departed from the medium, and therefore do not feel any transverse momentum kicks.
} 
model to this point, no effects of such a wake are included. We are in effect making the assumption that all the energy lost by the jet is so fully thermalized that it ends up as a little bit more plasma or a little bit hotter plasma, becoming a distribution of soft thermal particles in the final state that is isotropic, uncorrelated with the jet direction. This cannot be the whole story since, after all, momentum is conserved and the jet loses momentum to the plasma as well as energy. We shall return to this in section 4.

\subsection{An observable that is sensitive to broadening}

Before returning to the question of where the momentum and energy lost by the jet ends up and how this affects angular observables like the jet shape, we close this section by identifying a more differential observable that is directly sensitive to transverse momentum broadening, meaning to the value of the broadening parameter $K$.

Let us recapitulate why the observables that we looked at above are not sensitive to broadening. Kicks received by the few highest momentum partons in a jet contribute to the acoplanarity, as we have discussed, but because these partons have such a high momentum the effects on the acoplanarity are quantitatively very small. The conventional jet shape observable at larger values of $r$ is dominated by very soft particles. In our hybrid model as formulated in this section, these softest particles tend to originate from the last fragmentation events in the shower, as harder partons fragment after they have already left the medium. Soft partons that are produced earlier, in the medium, rapidly lose all their energy. Soft partons at large $r$ that are produced outside the medium cannot be affected by broadening. For different reasons, therefore, both the acoplanarity (dominated by the few hardest partons) and the jet shape at large $r$ (dominated by soft partons which survive because they were produced late, after the shower exits the plasma) are insensitive to transverse momentum broadening.

The key is to focus on the angular distribution of the energy in the jet that is carried by particles in an intermediate interval of transverse momentum. We must focus on semi-hard partons that are sufficiently soft that they can be deflected significantly by the transverse momentum kicks that they receive from the medium but that are sufficiently hard that they survive propagation through the plasma and emerge from it. The new observable that we define is, in essence, a more differential version of the jet shape. Instead of determining the $r$ distribution of all the jet energy, we focus on the distribution of jet energy carried by particles in a certain interval of transverse momentum. And, we construct this observable using only the subleading jets in dijet pairs, specifically subleading jets with $p_{\mathrm{T}}^{\text {subleading }}>$ $30 \mathrm{GeV}$ in a dijet whose leading jet has $p_{\mathrm{T}}^{\text {leading }}>120 \mathrm{GeV}$ and where the two jets are separated by $\Delta \phi>5 \pi / 6$. We make this choice because jets in a sample of subleading jets have on average lost more energy than in a sample of leading or inclusive jets. (We have checked that if we use a sample of leading or inclusive jets, we do see the effects that we shall describe below but they are smaller in magnitude than in the sample of subleading jets.) We denote our new observable by $\Psi_{p_{\mathrm{T}}}^{\text {subleading }}(r)$, with

$$
\Psi_{p_{\mathrm{T}}}^{\text {subleading }}(r) \equiv \frac{1}{N_{\text {subleading jets }}} \frac{1}{\delta r} \sum_{\text {subleading jets }} \frac{\sum_{i \in r \pm \Delta r / 2 ; p_{\mathrm{T}}^{i, \text { track }} \in \text { range }} p_{\mathrm{T}^{i, \text { track }}}}{p_{\mathrm{T}^{\mathrm{jet}}}},
$$


where we take the particles that we include to be only those that have a transverse momentum in a specified range. We take this range to be $10 \mathrm{GeV}<p_{\mathrm{T}}<20 \mathrm{GeV}$, a choice whose motivation we describe below. (Note that we first find and reconstruct jets using all particles, in the standard fashion, and it is from the fully reconstructed jets we obtain $p_{\mathrm{T}}{ }^{\text {jet }}$ for the leading and subleading jets. After we have a sample of reconstructed subleading jets, we evaluate $\Psi_{p_{\mathrm{T}}}^{\text {subleading }}(r)$ using only the particles within the specified range $10 \mathrm{GeV}<p_{\mathrm{T}}<20 \mathrm{GeV}$.) We shall look at the observable $\Psi_{p_{\mathrm{T}}}^{\text {subleading }}(r)$ for angles up to $r<1$. Recent preliminary results regarding somewhat similar jet shape observables, shown in figure 12 of ref. [169], indicate that the measurement of $\Psi_{p_{\mathrm{T}}}^{\text {subleading }}(r)$ will require background subtraction. We have chosen to follow the procedure used in ref. [169]. We discretize the event in $(\Delta \eta, \Delta \phi)$ space, where $\Delta \eta$ and $\Delta \phi$ are distances from the jet axis in rapidity and azimuthal angle, with $|\Delta \eta|<2.5$, in bins of width $0.025 \times 0.025$, building a two-dimensional energy density distribution using only tracks with $p_{\mathrm{T}}$ in the desired range. We take reconstruction and background subtraction effects into account by smearing the jet energy, computing the $\phi$-dependent average energy density far away from the jet axis in rapidity by summing over all bins with $1.5<|\Delta \eta|<2.5$, for each value of $\phi$, in each event, and then subtracting this average long-range energy density from the energy density in each of the bins with $|\Delta \eta|<1.5$, for each value of $\phi$, in each event. After this background subtraction, we then construct the observable $\Psi_{p_{\mathrm{T}}}^{\text {subleading }}(r)$ by summing the energy lying in the annulus at a distance $\Delta r=\sqrt{\Delta \eta^{2}+\Delta \phi^{2}}$ from the jet. Finally, to maintain consistency with the definition of the standard jet shape we multiply $\Psi_{p_{\mathrm{T}}}^{\text {subleading }}(r)$ by the factor $\left\langle p_{\mathrm{T}}{ }^{\text {jet }} / \sum_{i=0}^{N_{\text {bins }}} p_{\mathrm{T}}{ }^{\text {track }}\left(r_{i}\right)\right\rangle$ in order to ensure that $\Psi_{p_{\mathrm{T}}}^{\text {subleading }}(r)$ is normalized to one.

In figure 6 we show the prediction of our hybrid model for the ratio of the new observable $\Psi_{p_{\mathrm{T}}}^{\text {subleading }}(r)$ in $\mathrm{PbPb}$ collisions to that in proton-proton collisions for several values of the broadening parameter $K$. We show results for $\Psi_{p_{\mathrm{T}}}^{\text {subleading }}(r)$ with $p_{\mathrm{T}}$ in the interval $10 \mathrm{GeV}<p_{\mathrm{T}}<20 \mathrm{GeV}$. The selection of these cuts follows from several requirements. We want the partons to be hard with respect to the medium temperature to ensure that no possible collective effects (including those we will focus on in section 4) can affect the measurement; this is safely achieved by the lower $p_{\mathrm{T}}$ cut of $10 \mathrm{GeV}$. We also want the partons to be energetic enough to survive propagation through enough plasma that they pick up some transverse momentum kicks, which is to say so that they can exhibit sensitivity to the broadening parameter $K$. This motivates trying even somewhat larger values of the lower $p_{\mathrm{T}}$ cut. From the other side, we want to choose an upper $p_{\mathrm{T}}$ cut so that the partons used in the definition of $\Psi_{p_{\mathrm{T}}}^{\text {subleading }}(r)$ do have their direction of propagation significantly altered by the transverse momentum that they pick up from the plasma. We have found that the range $10 \mathrm{GeV}<p_{\mathrm{T}}<20 \mathrm{GeV}$ serves our purposes well. However, we have also investigated $\Psi_{p_{\mathrm{T}}}^{\text {subleading }}(r)$ with $20 \mathrm{GeV}<p_{\mathrm{T}}<40 \mathrm{GeV}$ and this works almost as well. Unlike for the less differential observables described previously, we see from figure 6 that $\Psi_{p_{\mathrm{T}}}^{\text {subleading }}(r)$ shows significant sensitivity to the value of $K$. Indeed, as $K$ grows, the partons in this momentum range are more and more kicked out to angles further away from the center of the jet, populating the large $r$ region and depleting the region nearest to the jet axis $(r=0)$. 


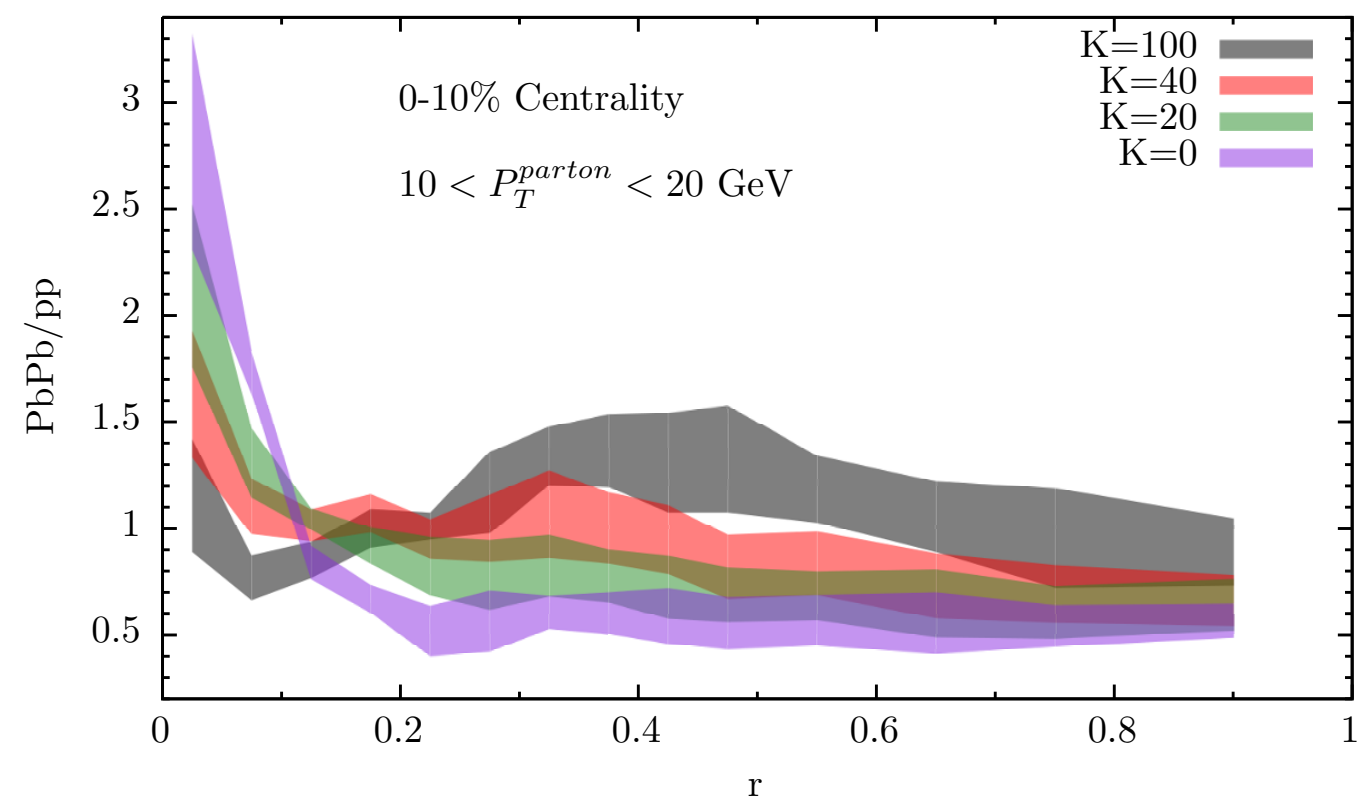

Figure 6. Differential jet shape ratio constructed from the subleading jets in dijet pairs satisfying $p_{\mathrm{T}}^{\text {leading }}>120 \mathrm{GeV}, p_{\mathrm{T}}{ }^{\text {subleading }}>30 \mathrm{GeV}$, and $\Delta \phi>5 \pi / 6$. The analysis only includes partons whose $p_{\mathrm{T}}$ lies within the intermediate range $10<p_{\mathrm{T}}<20 \mathrm{GeV}$. The effect of broadening on this observable is apparent.

The main features of the results plotted in figure 6 may be understood as coming from the dynamics of broadening and parton energy loss. Let us begin by looking at the $K=0$ curve. In the absence of any transverse momentum kicks, the dynamics of energy loss in the hybrid model depletes the number of partons at large angles $r$. This happens because these partons are produced early and are on average softer, and for both reasons they lose more energy in the plasma. This pushes the $\mathrm{PbPb}$ jet shape down at large $r$. Because the jet shape is normalized, it is pushed up at small $r$. Since there is no parton energy loss for the jets in pp collisions, the modification of the numerator in the jet shape ratio shows up directly in the $K=0$ jet shape ratio itself, plotted in figure 6 . Now let us ask what happens when we turn on $K \neq 0$, adding transverse kicks felt by all the partons in the jet as they propagate through the medium, namely broadening. As mentioned above, the depletion of partons at small angles $r$ from the jet axis due to broadening results in the reduction of the jet shape at small $r$ in $\mathrm{PbPb}$ collisions relative to that in pp collisions with increasing $K$. Since the shape of jets in vacuum is a rapidly falling function of $r$, kicking some partons from smaller $r$ to larger $r$ serves to enhance the jet shape in $\mathrm{PbPb}$ collisions, and hence pushes the jet shape ratio plotted in figure 6 upwards at larger $r$, again as a function of increasing $K$. Furthermore, as $K$ increases partons that are kicked from smaller $r$ can end up at larger and larger values of $r$, meaning that at larger and larger values of $K$ the increase in the jet shape ratio seen in figure 6 extends further and further to the right. These $K$-dependent effects are apparent in figure 6 , and they are of course the reason why we have selected and are highlighting this observable.

In experiment, of course, the range in $p_{\mathrm{T}}$ employed in the definition of $\Psi_{p_{\mathrm{T}}}^{\text {subleading }}(r)$ will need to be a range of momenta of final state hadrons, not a range of momenta of 


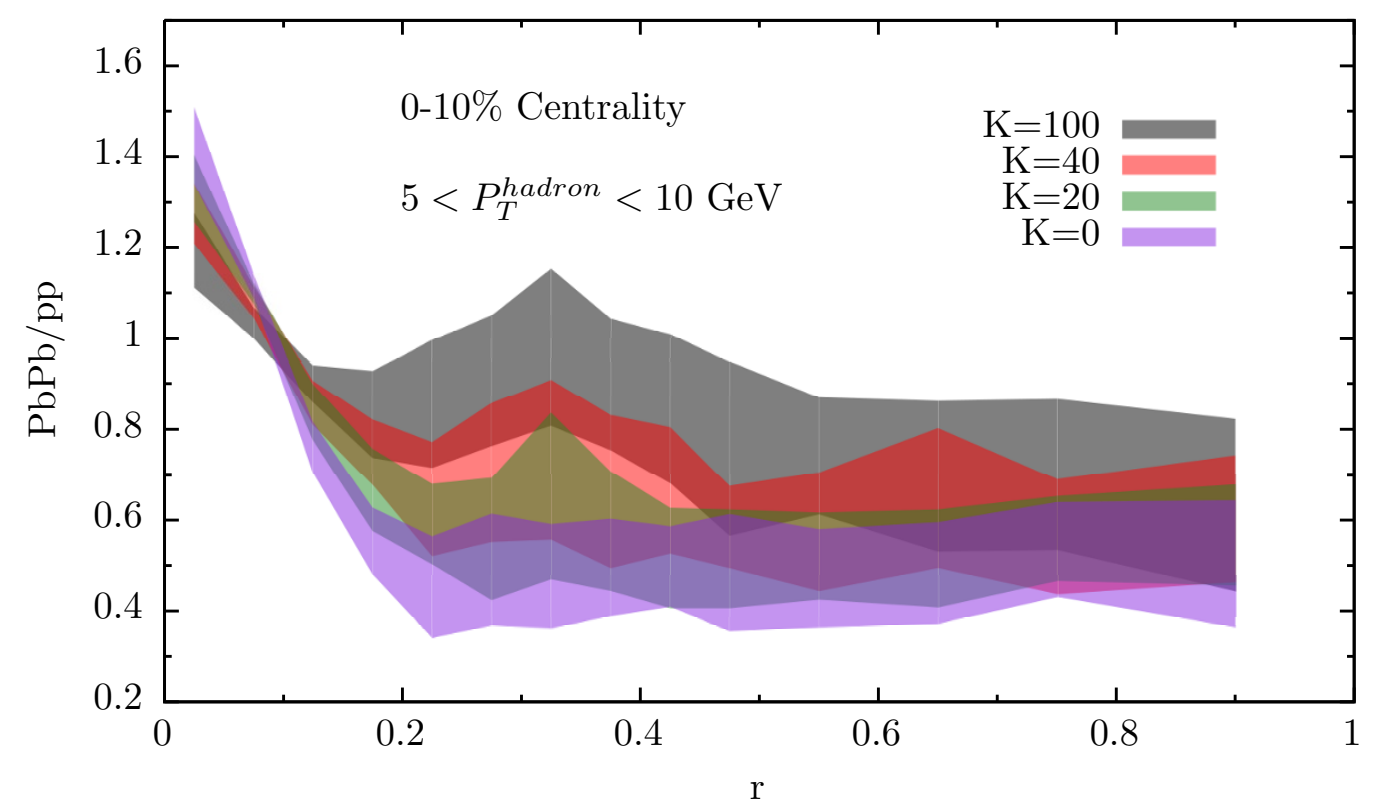

Figure 7. Differential jet shape ratio $\Psi_{p_{\mathrm{T}}}^{\text {subleading }}(r)$ constructed from charged hadrons with $5 \mathrm{GeV}<$ $p_{\mathrm{T}}<10 \mathrm{GeV}$, for subleading jets that satisfy the same cuts as in figure 6 .

partons. Further investigation of the effects of hadronization, which are not under good theoretical control, is clearly important. Nevertheless, the observed sensitivity to transverse momentum broadening that we see in figure 6 , and that we have not seen in any of the other observables we have studied, encourages us to explore hadronic versions of this observable that are sensitive to the angular distribution of the partons in jets with $10<p_{\mathrm{T}}<20 \mathrm{GeV}$ that contribute in figure 6 , with the goal of a direct extraction of the broadening parameter $K$, a key characteristic of the medium. In figure 7 we present the prediction from our hybrid model for a hadronized version of figure 6 , namely the observable $\Psi_{p_{\mathrm{T}}}^{\text {subleading }}(r)$ computed for hadrons rather than partons, with the specified range for the $p_{\mathrm{T}}$ of the hadrons in the analysis taken to be $5<p_{\mathrm{T}}<10 \mathrm{GeV}$. (We use the hadronization prescription described at the end of section 4.2.) We have selected a lower momentum range for the hadrons entering the analysis in figure 7 than for the partons in the analysis in figure 6 simply because hadronization turns partons into softer hadrons. Other choices of momentum range can be investigated.

The main features observed in figure 6 are also observed in figure 7 . As in the partonic case, broadening means that as $K$ increases we see a decreasing jet shape ratio at small $r$ and an enhancement in the intermediate $r$ region, with the region where enhancement is seen extending to larger $r$ as $K$ increases further. However, in the hadronic case, the separation between the curves with different values of $K$ values is less pronounced than in the partonic case. This is due, in part, to the fact that hadrons within any given range in momentum originate from partons with a wider range of momenta. The behavior of the $p_{\mathrm{T}}$-dependent partonic jet shape $\Psi_{p_{\mathrm{T}}}^{\text {subleading }}(r)$ is different in different $p_{\mathrm{T}}$ regions, and in particular it becomes less sensitive to $K$ for partons with very large momenta. Since 
some of the hadrons with momenta in the $5 \mathrm{GeV}<p_{\mathrm{T}}<10 \mathrm{GeV}$ range that we have used to construct the hadronic differential jet shape ratio in figure 7 come from the hadronization of partons with much higher momentum, this hadronic observable plotted in figure 7 shows less sensitivity to broadening than that seen in figure 6. Because hadronization can turn a single hard parton at very small $r$ into several softer partons that are more spread out in $r$, it tends to spread the largest changes seen at very small $r$ in figure 6 over a wider range of $r$ in figure 7, reducing their amplitude in the plot. Despite these effects which serve to dilute the $K$-dependence seen in the partonic observable, the hadronic observable in figure 7 displays sensitivity to transverse momentum broadening dynamics.

Although it is of course necessary to do further investigations of hadronization, it seems promising that by selecting hadrons from subleading jets in an intermediate $p_{\mathrm{T}^{-}}$ range, $5 \mathrm{GeV}<p_{\mathrm{T}}<10 \mathrm{GeV}$ in figure 7 , the effects of the transverse momentum kicks on the partons in the jet on the observable are enhanced, and are considerable, relative to what we have seen for less differential observables such as the usual jet shapes in figure 5 . The further investigation of observables that, like the $p_{\mathrm{T}}$-differential jet shape ratio we have constructed from subleading jets, focus on the transverse dynamics of partons in the jet that are neither very soft (as in that regime other effects that we investigate in the next section dominate) nor very hard (as in that regime the effects of transverse kicks received from the medium are hard to see) holds considerable promise. Such observables represent the only path that we can see at present toward an experimental determination of the value of $K$ directly from its definition in terms of momentum broadening rather than indirectly via inference from measurements of energy loss. And, $K=\hat{q} / T^{3}$ is a key parameter in our model or in any model as it quantifies a central property of the medium that is related to how strongly coupled the fluid is. We may not yet have hit upon the precise definition of the optimal observable, as doing so requires balancing choices of jet selection, $p_{\mathrm{T}}$ cuts, jet shape measure, and background subtraction in the face of potentially competing goals: maximizing sensitivity to $K$ while at the same time optimizing the statistics, signal-tonoise, and utility of the analysis of any specific data set. We look forward to seeing this done in consultation between experimentalists and theorists. Our results in figure 7 provide strong motivation for doing so.

\section{Medium backreaction: observable consequences of a wake in the plasma}

In this section we will take the first steps toward incorporating another important feature of jet quenching dynamics in plasma into our hybrid model: the conservation of the momentum and energy lost by the jet. In our implementation of the model up to now, we have assumed that the energy lost by the energetic partons in the jet thermalizes to such a complete extent that, after hadronization, it becomes particles moving in random directions that are completely uncorrelated with the jet direction. The assumption of rapid thermalization is well motivated by the many lines of evidence indicating that quark-gluon plasma is a strongly coupled liquid. It is also motivated by the holographic analysis of the stress tensor that describes the response of the $\mathcal{N}=4 \mathrm{SYM}$ fluid to the passage of an energetic parton through it $[123,125]$ which showed that after a short time of order 
$1 / T$ all the energy dumped into the medium was rapidly converted into a hydrodynamic excitations of the system. The possible hydrodynamic collective response of the plasma to the passage of an energetic parton through it was characterized earlier in refs. [171$174]$; the holographic computation in refs. $[123,125]$ provided a concrete realization in a full quantum field theoretical calculation, confirming that the anticipated hydrodynamic response could indeed be excited via the passage through the fluid of an energetic probe whose size is much smaller than $1 / T$. This notwithstanding, it cannot be correct to assume that the energy lost from the jet, after thermalization, has no memory of the direction of motion of the jet. After all, momentum is conserved and the jet loses momentum to the plasma, as well as energy. And indeed, the hydrodynamic response of the plasma includes both a wake and sound waves (a Mach cone) that carry momentum in the direction of the jet, as well as energy. In this section, we will provide an initial account of this collective response by providing a simplified description of the backreaction of the medium to the passage of the jet that respects energy and momentum conservation without introducing any additional parameters into our hybrid model. We leave a full treatment (which would involve sourcing, propagating, and hadronizing a hydrodynamic wake in the expanding cooling hydrodynamic fluid) to the future.

The mechanism we will describe is not exclusive to strong coupling, although this scenario provides a completely natural realization for this collective response. Even in perturbative QCD analyses in which a hard parton loses energy by radiating gluons the radiated gluons themselves interact with the medium and radiate further softer gluons, with the result being a rapid degradation of the emitted gluon momenta. This degradation may be viewed as a rapid transfer of energy from hard (jet) modes to soft (medium) modes and it has been described recently in detail in refs. [150-156]. In an infinite medium, this process leads to the eventual (local) thermalization of the lost energy after which the dynamics may be best understood in terms of hydrodynamics. In a finite medium, the local thermalization process may not be complete and it is possible that the medium modes excited by the jet may remember more than just the energy and momentum that the jet lost and they gained, but keeping track of their momentum and energy as we shall do is certainly a good first step. One circumstance in which our analysis in this section would not be relevant is for a medium that is sufficiently thin and sufficiently weakly coupled that gluons radiated by the hard parton do not interact after they are radiated, but this is an extreme scenario.

Following this discussion, in section 4.1 we will describe a simple implementation of the backreaction of the plasma to the passage of a jet of energetic partons through it. We will refer to the backreaction of the plasma in generic terms as the wake of the jet, as we shall not need to focus on the distinction between a diffusive wake (moving and perhaps heated fluid) and propagating sound waves since upon making the approximations that we describe below the perturbations to the spectrum of hadrons in the final state that we compute cannot distinguish between diffusive and sound modes. We will characterize the medium response to the transfer of momentum to it from the jet, that is the wake, by analyzing the induced velocity and temperature variations of the hydrodynamic behavior of the quark-gluon plasma fluid, upon making several simplifying assumptions. Since the 
total energy deposited in the medium by a typical jet in heavy ion collisions $(\mathcal{O} \sim 20 \mathrm{GeV})$ is small when compared to the total energy per unit rapidity in the event $(\mathcal{O} \sim 1 \mathrm{TeV})$, as in refs. [171-174] we shall treat the additional momentum acquired by the medium as a consequence of the passage of the jet as a small perturbation. (For studies of the nonlinear response of the plasma, see refs. $[158,161,175,176])$. We shall also assume that the small perturbation of the velocity and temperature of the medium translates into small perturbations to the resulting distribution of particles at all momenta. These physical assumptions can be described somewhat loosely by saying that we are assuming that the energy deposited into the medium as a wake thermalizes to the maximum degree allowed by conservation of momentum and energy, turning into a perturbation to the spectra of the hadrons in the final state that remembers the energy and momentum deposited by the jet into the medium but nothing else about its origin. As we have discussed, this is natural at strong coupling, or at weak coupling if the gluons radiated by the hard partons in the jet themselves radiate many times further, but would not be valid if the energy lost by the jet is carried by only a few particles.

For simplicity, we will also assume that the unperturbed fluid is well described by a boost invariant flow. And, although in our simulation of the amount of energy that the jet loses we do take the transverse flow of the medium into account and we do use a background obtained via solving viscous hydrodynamics, in this initial study of the wake we will for simplicity neglect the transverse flow of the unperturbed fluid and employ ideal hydrodynamics. All that said, our aim will actually not be a description of the perturbations of the hydrodynamics per se: we wish to focus instead directly on the modification to the spectrum of hadrons produced after the perturbed hydrodynamic fluid freezes out. The last simplifying assumption that we make is that the plasma freezes out along a constant proper time hypersurface.

After making these various approximations, in section 4.1 we will derive a simple expression for the modification to the spectrum of hadrons formed as the hydrodynamic fluid perturbed by the passage of a jet through it freezes out, an expression that is determined solely by the amount of energy and momentum lost by the jet. In subsequent subsections, we will describe how we implement this expression in our hybrid model, and look at its consequences for a number of jet observables.

It is inevitable that when a jet reconstruction algorithm is used to find and reconstruct jets in heavy ion collisions, some of the energy and momentum that is counted as part of a jet in fact comes from hadrons formed from the plasma as it freezes out, given that the plasma includes a moving heated wake that, by momentum conservation, is flowing in the same direction as the jet [95, 158, 160-162]. Furthermore, since any background subtraction procedure involves comparing events with a jet or jets to events that do not contain jets, and since events that do not contain jets also do not contain wakes, the particles from the hadronization of the wake that end up reconstructed as part of a jet will not be removed by background subtraction. Since there is no way for experimentalists to remove them from the jets they reconstruct, theorists must add them to the jets in their calculations. This is our goal in this section. 


\subsection{The spectrum of hadrons from a medium perturbed by the passage of a jet}

In a boost invariant fluid with no transverse velocity, the wake associated with the passage of a jet may be characterized by a perturbation to the velocity field with the form

$$
\delta u^{\mu}=\left(0, \delta u^{i}, \delta u^{\eta}\right),
$$

where $\delta u^{i}$ with $i=1,2$ and $\delta u^{\eta}$ are the variations of the velocity field in the transverse plane and in the space-time rapidity direction. The disturbance will also be associated with a change in the temperature of the plasma $\delta T$. These perturbations are functions of time and of all the space coordinates, and need not be boost invariant. (Note that because $u^{\mu}+\delta u^{\mu}$ is normalized, the $\delta u^{\tau}$ component in (4.1) is fixed by, and quadratic in, $\delta u^{i}$ and $\delta u^{\eta}$ and we have therefore neglected it.) At a fixed proper time $\tau$, the total momentum stored in the perturbation of the plasma is

$$
\Delta P_{\perp}^{i}=w \tau \int d^{2} x_{\perp} d \eta \delta u_{\perp}^{i}, \quad \Delta P^{\eta}=w \tau \int d^{2} x_{\perp} d \eta \delta u^{\eta},
$$

where we have used the fact that, to leading order in the perturbation, the variation of the stress tensor of ideal hydrodynamics takes the form $\delta T_{a}^{\tau}=w \delta u_{a}$ for $a=1,2, \eta$ with $w=\varepsilon+P$ is the enthalpy of the unperturbed fluid, which is related to the entropy density of the unperturbed fluid through $w=T s$ and which is constant on a fixed- $\tau$ surface in a fluid which is boost invariant and has no transverse expansion. Because we are simplifying this analysis by assuming ideal hydrodynamics, we are neglecting any production of entropy during the hydrodynamic evolution, for example as the sound waves excited in the plasma are damped by viscosity. The only entropy dumped into the plasma is that dumped into the wake initially by the jet itself, and this entropy is then equal to the entropy associated with the perturbation computed at late time by computing the flux of the entropy current across a fixed $\tau$ hyper-surface

$$
\Delta S=\frac{s \tau}{c_{s}^{2}} \int d \eta d^{2} x_{\perp} \frac{\delta T}{T}
$$

where $c_{s}$ is the speed of sound of the unperturbed fluid which, within the boost invariant assumption, only depends on the proper time $\tau$.

Both eqs. (4.2) and (4.3) are valid on any fixed proper time hypersurface including, in particular, on the freeze-out hypersurface. These expressions describe all the momentum and entropy that was dumped into the plasma by the disturbance, as they are at the freezeout time. Following our assumption that all the momentum lost by the jet is incorporated into the plasma as a wake, $\Delta P_{\perp}^{i}$ and $\Delta P^{\eta}$ are given by the transverse momentum and momentum rapidity lost by the jet as it traverses the plasma, respectively. Since in our implementation of energy loss we have assumed that the rapidity of the jet remains constant (or almost constant if we turn on transverse momentum broadening) we set $\Delta P^{\eta}=0$ and use our hybrid model calculation to give us the $\Delta P_{\perp}^{i}$ injected into the hydrodynamic fluid by each of the jets we analyze. We shall see below that the entropy production $\Delta S$ associated with the jet passage through the medium may also be constrained by the total energy carried by the particles produced by the perturbation. 
Our goal is to determine the effect of the perturbations $\delta u^{a}$ and $\delta T$ that describe the wake in the fluid that the passage of the jet creates on the spectrum of particles produced when the fluid freezes out. To do so, we will employ the standard Cooper-Frye prescription [177]

$$
E \frac{d N}{d^{3} p}=\frac{1}{(2 \pi)^{3}} \int d \sigma^{\mu} p_{\mu} f\left(u^{\mu} p_{\mu}\right)
$$

where the $\sigma_{\mu}$ integral is over the freeze-out hypersurface and where for simplicity we shall assume a Boltzmann distribution $f(E)=\exp (-E / T)$. Expanding to leading order in the perturbation, we obtain the expression

$$
\begin{aligned}
E \frac{d \Delta N}{d^{3} p}= & \frac{\tau}{(2 \pi)^{3}} \int d^{2} x_{\perp} d \eta m_{T} \cosh (\mathrm{y}-\eta) \exp \left[-\frac{\mathrm{m}_{\mathrm{T}}}{\mathrm{T}} \cosh (\mathrm{y}-\eta)\right] \\
& \times\left\{p_{\perp}^{i} \frac{\delta u_{\perp}^{i}}{T}+\tau^{2} p^{\eta} \frac{\delta u^{\eta}}{T}+\frac{m_{T}}{T} \frac{\delta T}{T} \cosh (\mathrm{y}-\eta)\right\}
\end{aligned}
$$

where $m_{T} \equiv \sqrt{m^{2}+p_{T}^{2}}$ is the transverse mass of the emitted thermal particle. Note that this perturbative expression is only valid for particles whose momenta $p$ are comparable to the temperature $T$ on the freeze-out surface, where the small perturbations in the hydrodynamic quantities results in a small perturbation on the resulting distribution of particles. For momenta far above $T$, where the thermal distributions are exponentially small, the perturbations in the hydrodynamic quantities can have large relative effects; in this regime, the expression (4.5) is not valid and, because it is based on a linear approximation to an exponential, it in fact underestimates the particle production from the wake. However, this regime of the spectrum contributes little to overall yields.

The expression in eq. (4.5) is general and independent of the space-time dependence of the perturbed hydrodynamic fields. To proceed further we will assume that during the space-time evolution of the perturbation over the boost invariant background, the spacetime rapidity of the disturbance remains approximately constant. Since high-energy jets propagate at a fixed space-time rapidity $\eta_{j}$ equal to their momentum rapidity $y_{j}, \eta_{j}=y_{j}$, this assumption implies that the perturbation is narrow around the momentum rapidity of the jet, which allows us to perform the $\eta$ integration in eq. (4.5) by replacing $\eta \rightarrow y_{j}$. We perform this integration, use eqs. (4.2) and (4.3) to relate the three terms in eq. (4.5) to $\Delta P_{\perp}^{i}, \Delta P^{\eta}$ (which vanishes) and $\Delta S$, and then impose that the energy and momentum of the emitted particles equals the energy and momentum lost by the jet

$$
\Delta E=\int d^{3} p \frac{d \Delta N}{d^{3} p} E, \quad \Delta P_{\perp, i}=\int d^{3} p \frac{d \Delta N}{d^{3} p} p_{\perp, i},
$$

where the integration of the first of these equations leads to the relation $\Delta S=$ $\Delta E /\left(T \cosh y_{j}\right)$. After these manipulations, we are able to express the spectrum of particles eq. (4.5) emitted from the boosted, heated up, wake in the fluid as

$$
\begin{aligned}
E \frac{d \Delta N}{d^{3} p}= & \frac{1}{32 \pi} \frac{m_{T}}{T^{5}} \cosh \left(\mathrm{y}-\mathrm{y}_{\mathrm{j}}\right) \exp \left[-\frac{\mathrm{m}_{\mathrm{T}}}{\mathrm{T}} \cosh \left(\mathrm{y}-\mathrm{y}_{\mathrm{j}}\right)\right] \\
& \times\left\{p_{\perp} \Delta P_{\perp} \cos \left(\phi-\phi_{j}\right)+\frac{1}{3} m_{T} \Delta M_{T} \cosh \left(\mathrm{y}-\mathrm{y}_{\mathrm{j}}\right)\right\} .
\end{aligned}
$$


where $p_{T}, m_{T}, \phi$ and $y$ are the transverse momentum, transverse mass, azimuthal angle and rapidity of the emitted thermal particles whose distribution we have obtained, and where $\Delta P_{T}$ and $\Delta M_{T}=\Delta E / \cosh y_{j}$ are the transverse momentum and transverse mass transferred from the jet (whose azimuthal angle and rapidity are $\phi_{j}$ and $y_{j}$ ) to the wake in the fluid. Note that the distribution (4.7) is a small correction that must be added to the one-body distribution of particles that the unperturbed hot plasma would have emitted. In particular, the distribution (4.7) may be negative. For example, this occurs for particles emitted in the direction opposite to the direction of the jet. Negative values simply mean that the perturbed thermal fluid emits less particles in the direction opposite to the direction in which the jet was propagating than the unperturbed fluid would have. This is a direct consequence of the fact that the jet loses momentum to the fluid, exciting a wake of fluid moving with net momentum along the direction of the jet.

The closed form expression (4.7), which only depends on the momentum lost by the jets in the plasma and on the kinematics of the jet, will be the basis for our analysis of the observable consequences of the wake in the plasma, which is to say of the backreaction of the medium. Subject to the assumptions employed in its derivation, the spectrum (4.7) will allow us to provide estimates of the observable effects of the collective response of the plasma to the passage of the jet through it without having to model the complicated processes (pre-hydrodynamic and hydrodynamic) via which the energy lost by the jet relaxes. As stated above, even within the assumptions employed in its derivation the expression (4.7) is only valid for particles emitted with a momentum comparable to the mean thermal momentum in a fluid cell at freeze-out; it need not be valid for semi-hard particles produced in the plasma. Perhaps the most important assumption in its derivation is the assumption that the wake in the plasma is fully thermalized, with the only aspects of its origins that it remembers being its total energy and momentum. This too need not be valid for semi-hard particles, some of which will originate from the energy lost by the jet either near the edge of the plasma or shortly before freezeout that do not thermalize. Nevertheless, we will use the spectrum (4.7) at all momenta to obtain first estimates of the observable consequences of the presence of a wake in the plasma in events with jets. In the next subsection, we shall explain how we have implemented this spectrum in our hybrid model analysis of jets propagating within hot QCD plasma.

\subsection{Implementation of backreaction, background model, and jet hadronization}

The implementation of the simplified expression (4.7) for the spectrum of particles resulting from the wake that is the backreaction of the medium to the presence of the jet demands further modelling for a proper description in heavy ion collisions. Three aspects that we will need to incorporate into our description are: (i) the effect of the radial flow and chemical composition of the unperturbed fireball on the particles resulting from the backreaction perturbation; (ii) adding a background of particles coming from the freeze-out of the unperturbed fireball to our hybrid model in order to be able to properly account for the negative contribution from the perturbation (4.7); and (iii) the generation of particles consistent with the one-body distribution eq. (4.7). Furthermore, since the particles produced after decoupling are hadrons, we will also need to consider the hadronization of our 
quenched jets, which we have not needed to include in our previous implementation of the hybrid model. We will discuss these four aspects sequentially in this subsection.

As we have stated, our derivation of eq. (4.7) neglects the effect of transverse flow in the unperturbed fluid, meaning that we have neglected radial flow, as well as elliptic flow and higher azimuthal harmonics of the transverse flow. The effects of elliptic flow and higher harmonics are small in the most central collisions, but radial flow cannot be neglected as it has important consequences for the spectrum of particles produced by the fireball. The radial boost in the spectrum of particles due to the radial flow in the fluid from which they are formed yields a blue-shifted spectrum which is harder than in the absence of the boost. Since heavier particles pick up more momentum than lighter particles when all are boosted by the same velocity, another consequence of radial flow is differing spectra for particles with different masses, with heavier hadrons getting harder spectra than lighter hadrons. The result (4.7) was derived as a perturbation on a background that does not include either effect. As a crude step towards including both effects, we will employ the spectrum eq. (4.7) but instead of using the temperature $T$ at the time of freezeout we will use species-dependent, momentum-dependent, empirical expressions for $T$ that provide a good description of the measured particle spectra in ref. [178] upon fitting the measured spectra to "thermal" spectra without radial flow. Specifically, we assume a proton to pion ratio of 0.05 , neglect hadrons other than protons and pions, and use the following momentum-dependent "temperatures" in eq. (4.7):

$$
\begin{aligned}
& T_{\pi}\left(p_{\mathrm{T}}\right)= \begin{cases}0.19 \mathrm{GeV} & \text { if } p_{\mathrm{T}}<0.7 \mathrm{GeV} \\
0.21\left(\frac{p_{\mathrm{T}}}{\mathrm{GeV}}\right)^{0.28} \mathrm{GeV} & \text { if } p_{\mathrm{T}}>0.7 \mathrm{GeV}\end{cases} \\
& T_{p}\left(p_{\mathrm{T}}\right)= \begin{cases}0.15 \mathrm{GeV} & \text { if } p_{\mathrm{T}}<0.07 \mathrm{GeV} \\
0.33\left(\frac{p_{\mathrm{T}}}{\mathrm{GeV}}\right)^{0.3} \mathrm{GeV} & \text { if } 0.07 \mathrm{GeV}<p_{\mathrm{T}}<1.9 \mathrm{GeV} \\
0.4 \mathrm{GeV} & \text { if } p_{\mathrm{T}}>1.9 \mathrm{GeV}\end{cases}
\end{aligned}
$$

These empirical expressions provide a good description of both the proton and pion spectra in central heavy ion collisions with $\sqrt{s}=2.76 \mathrm{TeV}$ at the LHC for $p_{\mathrm{T}}<3 \mathrm{GeV}$. (The largest deviations, around $10 \%$, occur for $p_{\mathrm{T}} \lesssim 0.5 \mathrm{GeV}$, where the measured pion yields contain large contributions from resonance decays.) We shall use them in eq. (4.7) for $p_{\mathrm{T}}<5 \mathrm{GeV}$. This approach is admittedly crude, but it is of value in this exploratory investigation as it allows us to use the closed form expression (4.7) rather than attempting a full hydrodynamic calculation of the wake induced by each of the jets in our ensemble of events.

As we have already discussed, a characteristic feature of eq. (4.7) is that the spectrum of particles coming from the perturbation, from the wake in the fluid, can become negative at large azimuthal angles with respect to the jet direction, particulary for particles with small $m_{T}$ or for jets with small $\Delta M_{T}$. This reflects the fact that the wake is made up of fluid moving with a net momentum in the jet direction, with the negative contribution to the perturbation representing a depletion of the momentum in directions opposite to that of the jet. This means that in order to implement eq. (4.7) in our hybrid model, we need to embed our jet sample in a background whose purpose is to provide sufficient thermal particles such that where the perturbation (4.7) is negative we have some thermal 
particles that we can remove. In previous implementations of our hybrid model in which we neglected the back reaction of the medium in response to the presence of a jet we ignored the background on the basis that in experimental analyses of jet data it would be subtracted. Contributions from the wake cannot be subtracted, which is why we are now adding them. Since these perturbations can be negative, we now need a baseline background too. The background that we use is oversimplified. It is constructed by generating an ensemble of pions and protons which is flat in $\phi$ and $\eta$ that reproduces the measured particle yields and spectra [178]. The addition of this contribution will force us to introduce a background subtraction procedure in our analysis of in-medium jets. We will describe this procedure in detail in subsection 4.3 and appendix B

For each event in our sample, we determine the momentum lost by each of the partons in the jet shower as well as in initial state radiation following our hybrid strong/weak coupling model as described in section 2. (For the backreaction analysis, we include all partons in the jet shower and in initial state radiation, whether or not they end up being reconstructed in a jet.) Since each of the propagating partons loses energy into the medium, each of them generates its own wake induced by its lost momentum. In the linearized approximation that we have employed, the multiple wakes do not alter each other, and the final spectrum is the superposition of the spectra generated by the wake of each propagating parton. At hadronization, each of the induced wakes generates an ensemble of particles with the one body distribution (4.7) computed from the kinematics of each parton and its lost momentum.

In order to incorporate the effect of fluctuations in the reconstructed jets, we generate the ensemble of particles coming from the wake in the medium via a simple Metropolis algorithm designed to satisfy the conservation of the lost jet energy while drawing particles from the distribution (4.7). First we generate an independent list of particles, including protons with $5 \%$ probability and pions with $95 \%$ probability, from the one body distribution (4.7), until the sum of their energies reaches the lost energy. This ensemble generally contains particles in the region of azimuthal angle in which eq. (4.7) becomes negative, which we will call negative particles. Whenever a negative particle is produced, its contribution to the net energy and momentum of the ensemble is negative. We later neutralize these negative particles by removing a particle from the background which is sufficiently close in $(\eta, \phi)$ space and in transverse energy. ${ }^{2}$ From this initial ensemble, whose fourmomentum in general will not coincide with the momentum lost by the jet, we randomly select a particle which we replace by a new particle drawn from the distribution (4.7). If

\footnotetext{
${ }^{2}$ Negative particle neutralization proceeds as follows. We identify the background particles within an angle of $\Delta r=0.3$ in $(\eta, \phi)$ space of the negative particle we wish to neutralize. Among the candidate background particles, we choose the best candidate in terms of $E_{T}$ and angular position in $\eta-\phi$ plane, which means we minimize $\Delta E_{T}$ and $\Delta r$, the difference in transverse energy and angular position with respect to that of the negative particle, respectively. We do the minimization by starting with any one of the candidate background particles and then checking each one of the other candidates to see whether choosing it instead reduces $\Delta E_{T}$ while not increasing $\Delta r$ by more than 0.05 or reduces $\Delta r$ while not increasing $\Delta E_{T}$ by more than $0.1 \mathrm{GeV}$. We have checked that after using this procedure we end up with $90 \%$ of the negative particles neutralized to better than $0.1 \mathrm{GeV}$ in transverse energy via subtraction of a background particle that was within $\Delta r=0.13$ of it.
} 
the change improves four-momentum conservation, it is accepted. Otherwise, the change may be accepted with a probability distribution

$$
W\left(p_{\text {new ensemble }}^{\mu}\right)=\frac{e^{-\left(p_{\text {new ensemble }}^{\mu}-\Delta P^{\mu}\right)^{2}}}{e^{-\left(p_{\text {ensemble }}^{\mu}-\Delta P^{\mu}\right)^{2}}},
$$

where $p_{\text {new ensemble }}^{\mu}$ is the four momentum of the candidate ensemble including the newly drawn particle, $p_{\text {ensemble }}^{\mu}$ the four-momentum of the previous ensemble, and $\Delta P^{\mu}$ is the four-momentum lost by the jet. We repeat the procedure until each of the four components of $p_{\text {ensemble }}$ is within $0.4 \mathrm{GeV}$ of the momentum lost by the jet. (We have explicitly checked that changing this threshold does not significantly change our results.) The ensemble generated after this procedure conserves energy and momentum (within the tolerance above) and possesses a one-body distribution identical to eq. (4.7), something that we have checked explicitly.

Since the medium response manifests itself in the form of modified distributions for hadrons, namely pions and protons in our approach, we are forced to consider the hadronization of jet showers in order to properly incorporate the particles from the jet itself and those from its wake in each event before then reconstructing jets and calculating observables. Hadronization leads, generically, to a softening of the typical jet fragments. Because of its nonperturbative nature, hadronization even in vacuum remains a fundamental problem and presents serious challenges to phenomenological modelling. Furthermore, it is not presently understood how the phenomenological hadronization models that have been applied successfully to QCD processes in vacuum should be modified due to the presence of a heavy ion environment. As an example, changes in how color flows in the jet shower resulting from soft exchanges between partons in the shower and the medium lead to significant modifications to subsequent hadronization in certain regions of phase space [179, 180], but the overall description of these effects remains to be determined. Because of all these uncertainties, in this work we will adopt a simplified model for the inmedium hadronization, as in much of the literature. We simply assume that hadronization of high energy jets occurs in heavy ion collisions as in vacuum, in particular keeping the same color correlations between partons in the shower even though we know that in reality these must change as the partons interact with the medium. Although several different prescriptions for hadronization in vacuum exist, in this work we will employ the Lund string model as implemented in PyтнIA, feeding the showers to this hadronization model after they have been modified according to our hybrid approach to energy loss and broadening. We defer comparisons between different models for hadronization to future work.

\subsection{The effect of backreaction on jet observables}

After the implementation of the energy loss suffered by the partons in jet showers produced in hard processes as they propagate through the hydrodynamic medium via the hybrid model described in section 2, the incorporation of the effects of transverse momentum broadening described in section 3 if we choose $K \neq 0$, the incorporation of a thermal-like background of particles and the perturbation to that background corresponding to the 
effects of the wake in the hydrodynamic medium as it responds to the passage of the jet as described in subsection 4.2, we now have a full event simulation from which to extract calculations of medium-modified jet observables. This will be our goal in this and the next subsections. In contrast to our previous publications, the inclusion of a background forces us to implement a background subtraction procedure, making our analyses of quenched events more similar to the actual analysis of jet data at the LHC. The fact that the medium responds to the passage of the jet, and in particular the fact that the medium picks up momentum in the jet direction, makes this complicated procedure absolutely necessary.

For hard jets produced together with a soft background that is completely uncorrelated with the jet direction, there are a number of established techniques that allow for systematic removal of the effects of background particles from jet observables. (See, for example, refs. [181-183].) These procedures, generically referred to as background subtraction, are routinely applied to jet measurements at the LHC and, at least in proton-proton collisions, they efficiently remove the effects of soft (non-perturbative) backgrounds that may be large but that are uncorrelated with the jet, allowing the measurement of theoretically controlled hard processes. However, in heavy ion collisions the fact that the medium includes a wake that carries momentum in the jet direction means, in effect, that a component of the background is correlated with the jet direction. This makes it impossible for a background subtraction procedure to separate the jet (which has been modified, via energy loss and broadening) from the medium (which has been modified, via the wake). In order to compare to experimental measurements, therefore, we have added a background and a wake and must now perform a background subtraction as if the background were uncorrelated with the jet direction, followed by jet reconstruction, just as in an experimental analysis. This procedure is not necessary for jet observables that are dominated by the harder components of a jet. This procedure is important for the softer components, since the softer components of what is reconstructed as a jet will include contributions from the jet itself and from the wake in the medium. In particular, this procedure is critical to gauging the effects of the wake on observables. We have implemented a full background subtraction procedure to analyze the events produced within our framework. In particular, we have implemented a version of the so called noise/pedestal background subtraction procedure $[48,184]$ and then done a jet energy scale correction; the details of our implementation can be found in appendix B.

As in section 3 , we first consider the $R$-dependence of jet suppression. In figure 8 we show the jet $R_{\mathrm{AA}}$ as a function of $p_{\mathrm{T}}$ for central events for a wide range of jet momenta and for different anti- $k_{t}$ reconstruction parameters $R$. We set $K=0$, neglecting transverse momentum broadening since, as we saw in section 3, it has little effect on these observables. In the right panel, we show our results for $70<p_{\mathrm{T}}<300 \mathrm{GeV}$ in comparison with CMS measurements of $R_{\mathrm{AA}}$ for $R=0.3$ jets in the range $100<p_{\mathrm{T}}<300 \mathrm{GeV}$. Note that, as when we included broadening in section 3 , when we incorporate the effect of the backreaction of the medium to the jet this alters the jet suppression, meaning that we had to retune the energy loss parameter $\kappa_{\mathrm{sc}}$ in our hybrid model. In this case, we only needed to modify the value of $\kappa_{\mathrm{sc}}$ at the percent level, which is very much smaller than the theoretical 

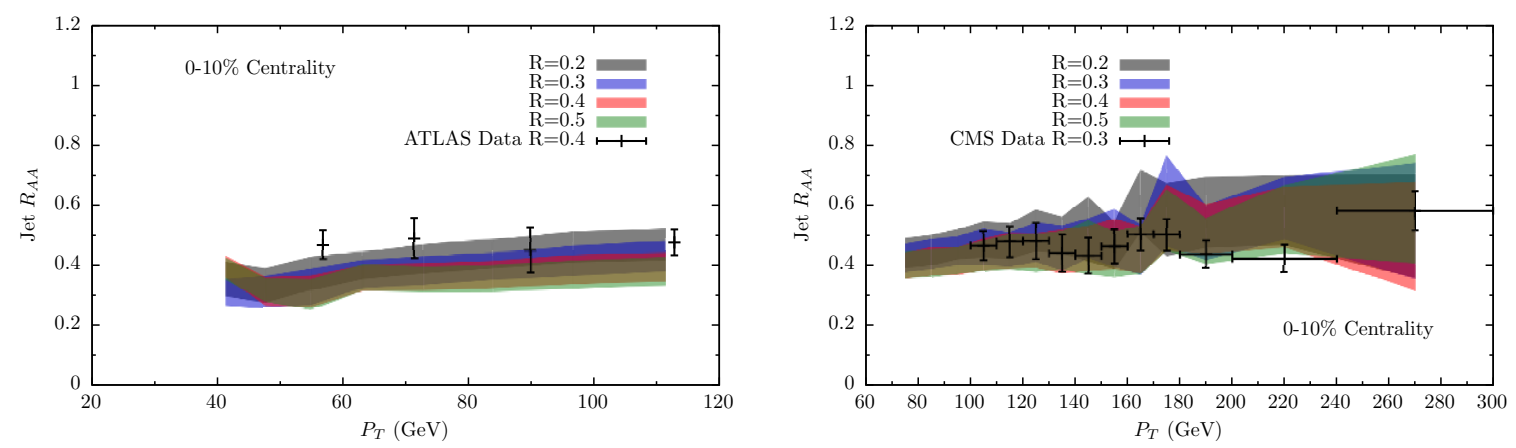

Figure 8. Dependence of jet $R_{\mathrm{AA}}$ on the anti- $k_{t}$ parameter $R$ used in reconstructing the jet and on the transverse momentum of the jet. We have set $K=0$, turning off transverse momentum broadening which we saw in section 3 has only very small effects on this observable. We have included the effects of the wake in the hydrodynamic fluid, which is to say the backreaction of the medium to the presence of the jet, and have added a background and implemented two different background subtraction procedures as described in the text and in appendix B. The left plot shows this observable for jets with $40<p_{\mathrm{T}}{ }^{\text {jet }}<120 \mathrm{GeV}$ as measured by the ATLAS collaboration [56], with their background subtraction procedure, while the right one is for jets with $70<p_{\mathrm{T}}{ }^{\text {jet }}<$ $300 \mathrm{GeV}$ as measured by the CMS collaboration [49], with their background subtraction procedure. Both panels are for the $0-10 \%$ most central collisions with $\sqrt{s}=2.76 \mathrm{ATeV}$. We have extended the $p_{\mathrm{T}}$-range in the right panel down to $70 \mathrm{GeV}$ even though present CMS measurements are for jets in the range $100<p_{\mathrm{T}}{ }^{\text {jet }}<300 \mathrm{GeV}$ to make it possible to compare the results from our model in the $p_{\mathrm{T}}$-range where the two panels overlap in order to see the effect on $R_{\mathrm{AA}}$ of choosing between the two different background subtraction procedures. The difference between the two panels is small, but visible.

uncertainty corresponding to the widths of the bands in all our plots in this paper and in our previous publications. As we found when we included broadening in section 3 , for the high energy jets in the right panel of figure 8 the suppression factor $R_{\mathrm{AA}}$ shows only a very small dependence on $R$, consistent with LHC data [49].

As we saw in section 3 , the suppression factor $R_{\mathrm{AA}}$ shows a small decrease (i.e. increase in suppression) with increasing $R$, corresponding to the fact that with increasing $R$ the angular size of the jets that are reconstructed increases together with the fact that wider jets lose more energy. However, this effect is milder here than it was in figure 2 because the effect of the backreaction of the medium that we are incorporating here is that some particles coming from the wake in the plasma, which is to say some of the energy that the jet lost, ends up reconstructed as if it were still part of the jet. Nevertheless, the wide angular distribution of the spectrum of particles from the wake given by eq. (4.7) implies that, even for the relatively large value of $R=0.5$ explored in figure 8 , the fraction of the energy lost by the jet that is recovered by the reconstruction procedure is small. For even larger values of the reconstruction parameter $R$, the full jet energy would in principle be recovered and $R_{\mathrm{AA}}$ would approach unity. However, for such large values of $R$ the fluctuations in the background make the reconstruction algorithm we have implemented unreliable. (We have tested the recovery of jet energy by embedding our simulations in a homogeneous background with no fluctuations.) 


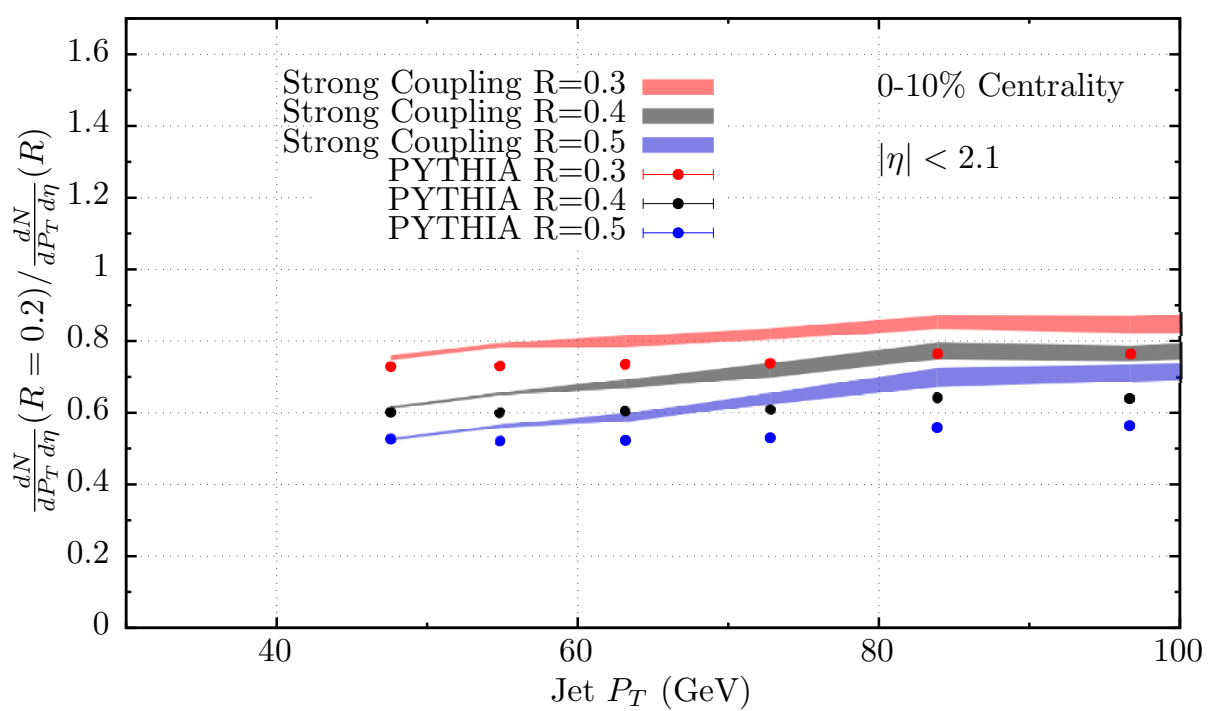

Figure 9. Ratio of the spectra of jets reconstructed with the anti- $k_{t}$ parameter $R=0.2$ to the spectrum of jets reconstructed with various larger values of $R$ as a function of the reconstructed jet $p_{t}$. The colored bands correspond to the prediction of our hybrid model, with no broadening $(K=0)$ and including the effects of the backreaction of the medium. The dots are the predictions for jets in vacuum, in proton-proton collisions as described by PYTHIA. As explained in the text, the fact that the three colored bands are closer together than the three sets of dots are is due to the fact that wider jets lose more energy than narrower jets do.

In the left panel of figure 8 , we show the jet $R_{\mathrm{AA}}$ for smaller jet $p_{\mathrm{T}}$ for the different values of $R$. The calculated suppression factor shows a mild increase with $p_{\mathrm{T}}$. It is consistent with all but the lowest $p_{\mathrm{T}}$ ATLAS data point within the theoretical and experimental uncertainties, although the data themselves don't show evidence for such an increase. In spite of the lower jet energy, the effect of including the particles coming from the backreaction of the medium on $R_{\mathrm{AA}}$ is small for all the values of $R$ we have explored, meaning that the $R$-dependence of our results for jet $R_{\mathrm{AA}}$ is similarly small to that seen in the right panel of the figure. This is in apparent contrast with $R_{\mathrm{CP}}$ measurements reported by ATLAS in ref. [48], which seem to indicate that the suppression of jets decreases with $R$ in this range of momenta, albeit with sizeable systematic uncertainties. Since ATLAS reports the $R$-dependence of $R_{\mathrm{CP}}$ (which can be thought of as the ratio of $R_{\mathrm{AA}}$ for central events to that for peripheral events) rather than of $R_{\mathrm{AA}}$, and since our simulations do not reproduce $R_{\mathrm{AA}}$ for peripheral events well (since the small amount of energy lost in the hadronic phase, which we do not include in our hybrid model, is proportionally more relevant in peripheral events where the total energy lost is smaller $[148,149])$ we will not attempt to make any quantitative comparisons with these data. We look forward to anticipated further measurements of the $R$-dependence of $R_{\mathrm{AA}}$ itself, where a direct comparison will be possible.

To further study the $R$-dependence of jet suppression in our model, and motivated by the ALICE analysis reported in ref. [58], in figure 9 we show the ratio of spectra of jets in $\mathrm{PbPb}$ collisions reconstructed with different values of the anti- $k_{t}$ parameter $R$ to that 
for $R=0.2$ jets. For comparison, we show the same ratio in proton-proton collisions as simulated by PYTHIA. An important advantage of this observable is that it is constructed with $\mathrm{PbPb}$ data only. This leads to a significant reduction in the theoretical uncertainties in our model, since the errors in the spectra with different $R$ are correlated. This is the reason why the widths of the colored bands depicting our theoretical predictions displayed in figure 9 are significantly narrower than those for our predictions of $R_{\mathrm{AA}}$. Because of this reduction in the theoretical uncertainty, as we shall now explain this observable is sufficiently discriminating to show that, within the range of $R$ studied, the wider jets that are reconstructed with larger values of $R$ lose more energy than narrower jets. To see this, let us first understand the behavior of this observable for jets produced in proton-proton collisions, which evolve in vacuum. Predictions from PyтнIA for these jets are shown as the colored dots in figure 9 . In vacuum, the number of jets with a given $p_{\mathrm{T}}$ increases as $R$ increases, since reconstructing wider jets incorporates a larger fraction of the initial partonic energy into the jet. Therefore, the ratio of the spectra of jets reconstructed with $R=0.2$ to that of jets reconstructed with a given $R$ decreases with increasing $R$. In the medium produced in $\mathrm{PbPb}$ collisions, this general trend is also observed. However, this ratio decreases more slowly with increasing $R$, meaning that the number of jets with a given $p_{\mathrm{T}}$ now increases more slowly with increasing $R$ than was the case in vacuum. This means that the wider jets reconstructed with larger $R$ have lost more energy than the narrower jets have.

Another interesting feature of this observable is that the deviation between the vacuum and medium ratios increases as the momentum of the jet increases. This, too, is a consequence of the fact that wider jets which contain more in-medium partons lose more energy than narrower jets. Low $p_{\mathrm{T}}$ jets contain, on average, a smaller number of partons propagating simultaneously in medium. The extreme case is a jet that consists only of a single parton while it is in the medium, although it may branch later. In this case, the energy loss is independent of the reconstruction parameter $R$ : if this jet is reconstructed, its energy loss is the energy lost by that single parton no matter what the value of $R$. The fact that the three colored bands in figure 9 come closer together as $p_{\mathrm{T}}$ increases indicates that the lowest $p_{\mathrm{T}}$ jets are dominated by jets that contain only very few partons while they are in the medium. As the $p_{\mathrm{T}}$ increases, the population of jets includes some that are wider, with more partons, that lose more energy. And, at any given $p_{\mathrm{T}}$ more of these are reconstructed at larger $R$. Although a direct comparison with the semi-inclusive jet measurements performed by ALICE [58] that motivated us to make figure 9 is not possible, our analysis highlights the potential that precise measurements of this type will have to discern the mechanisms of jet quenching in future.

Paralleling the discussion in section 3, to explore the effects of medium-modification of jets on their angular structure we now turn to the jet shapes ratio, shown in figure 10 for two different centralities. We set $K=0$ since, as we saw in section 3 , transverse momentum broadening has little effect on this jet shapes ratio. We describe the way in which we subtract the thermal background in appendix B. Since the fluctuations in our simplified background do not coincide with those of an actual heavy ion collision, we need to correct for the difference in jet energy resolution in order to do a fair comparison with CMS data [51]. 

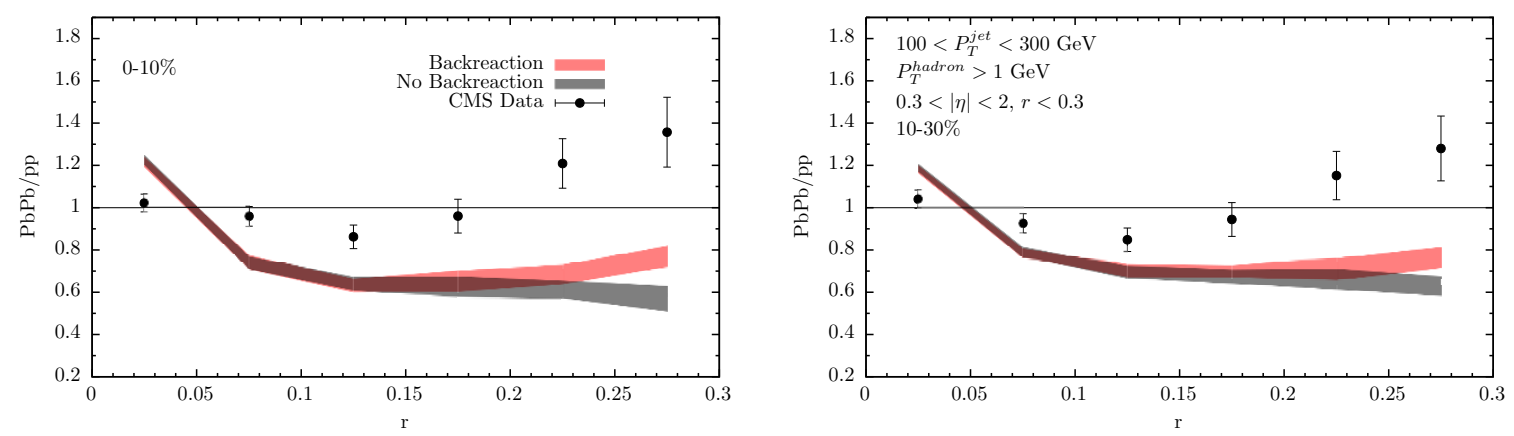

Figure 10. Ratio of the jet shape in $\mathrm{PbPb}$ collisions with $\sqrt{s}=2.76 \mathrm{ATeV}$ with $0-10 \%$ centrality (left) and 10-30\% centrality (right) to the jet shape in proton-proton collisions. The two colored bands show the results of our hybrid model calculation with no broadening, with both jets and background hadronized, and with our background subtraction procedure for high- $p_{\mathrm{T}}$ jets applied. In the calculation shown as the red band we include the effects of backreaction, namely the particles coming from a wake in the medium. We compare our calculation with and without backreaction to data from CMS [51].

This amounts to smearing the jet energies with a Gaussian whose width corresponds to the difference between the jet energy resolution in the presence of our background and the jet energy resolution measured by CMS; we describe the procedure in appendix B. Last, we subtract background tracks in the jet cone following a simple procedure from ref. [51] in which we subtract the $\eta$-reflection of each event from that event. This procedure does not work for jets near $\eta=0$; this is why $|\eta|<0.3$ is excluded from both our analysis and the measurement reported in [51].

To gauge the effects of adding our simplified background, performing the background subtraction procedure, and hadronization on one hand, and the effects due to the backreaction of the medium, namely the particles coming from the wake in the plasma, on the other in both panels we show the jet shape ratio computed at the hadronic level with and without backreaction. As we saw in section 4, energy loss serves to narrow the angular size of jets in a given window of energies in heavy ion collisions relative to that of jets with the same energies in proton-proton collisions. As a consequence, without backreaction the effect of energy loss is to increase the importance of narrow jets in the quenched jet sample, leading to a depletion of the jet shape at large angles $r$. Note that the only differences between the simulations without backreaction in figure 10 and the $K=0$ simulations displayed in figure 5 are: adding the simplified but fluctuating background that we are employing, performing our background subtraction and jet reconstruction, and adding hadronization. The partonic distributions whose ratio is plotted in figure 5 give rise to narrower distributions that the hadronic ones that go into figure 10, a natural consequence of the non-trivial angular distribution of the Lund strings connecting the hard partons within the jet which means that hadronization broadens the jet somewhat. (See for example ref. [185].)

Despite the hadronic uncertainties, the jet shape ratio shows a clear increase at larger values of the angular variable $r$ when we include backreaction, confirming the expectation that some of the particles from the wake in the plasma do end up reconstructed as part 
of the jet, and confirming the expectation that they are less tightly focused in angle than the jet itself was. That said, it is also clear from figure 10 that the data features a much stronger increase in the jet shape ratio at large angular distance $r$ from the jet axis than we obtain from our calculation. (Although the effect is large when plotted in this way, it is important to keep in mind that the enhancement in the ratio of jet shapes at large $r$ does not mean that in-medium jets are significantly wider than vacuum jets: in both populations, most of the jet energy is concentrated at small $r$; at large $r$ what is plotted is the ratio of two quantities that are both small. The data indicate that in $\mathrm{PbPb}$ jets the fraction of the jet energy at $r>0.2$ is larger than in proton-proton jets, but both are small.) Comparing the measured jet shape ratio to our calculations in figure 10 tells us that our treatment of backreaction substantially underestimates the amount of energy that ends up correlated with the jet axis but separated from it by a relatively large value of $r$. The hadronization of the wake in the hydrodynamic medium accomplishes this, but at least in our treatment it does not do enough. It could be that we are underestimating the amount of energy deposited in the medium at these angles, or it could be that we are missing energy at these angles that corresponds to energy lost by the jet that hydrodynamizes only partially or not at all, or it could be that our treatment of background fluctuations, background subtraction, and jet reconstruction is subtracting away more of the particles originating from the backreaction of the medium than happens in analyses of experimental data. And, as we will elaborate in section 5 , the jet shape analysis is sensitive to semi-hard particles in the region of $p_{\mathrm{T}}>2 \mathrm{GeV}$, in which the small-perturbation assumption behind eq. (4.7) starts to break down. We shall continue this discussion in section 5.

Next, we now turn from the angular jet shape variable to another intrinsically hadronic observable that assesses the longitudinal structure of jets: jet fragmentation functions. These show the distribution of the $z$ of the tracks in a jet, where $z \equiv p_{\text {track }} \cos \theta / p_{\text {jet }}$ is the ratio of the longitudinal momentum of a single charged hadron in the jet (a single track) to the momentum of the whole jet. ( $\theta$ is the angle between the track and the jet axis.) Unlike in our previous publications [148, 149], where we only analyzed partonic fragmentation functions, the inclusion of hadronization allows us to do a direct comparison with experimental measurements. However, we must keep in mind that the hadronization process is not under good theoretical control and that we have only implemented a simplified prescription which ignores changes in the color flow of jets that shower in a medium. We defer the study of effects of different prescriptions for hadronization in vacuum and in medium within our model to the future.

Results from our hybrid model calculations of jet fragmentation functions are shown in figure 11 for LHC heavy ion collisions with $0-10 \%$ and $10-30 \%$ centrality. Both panels display the ratio between hadronic fragmentation functions in $\mathrm{PbPb}$ collisions to those in proton-proton collisions compared to experimental measurements of this quantity by CMS [51]. We include two bands, one for the full calculation including the effects of the backreaction of the medium, and the other without it. The overall background subtraction and hadronization are the same in both calculations, as is the jet energy resolution correction and the subtraction of background tracks in the jet cone by $\eta$-reflection. 

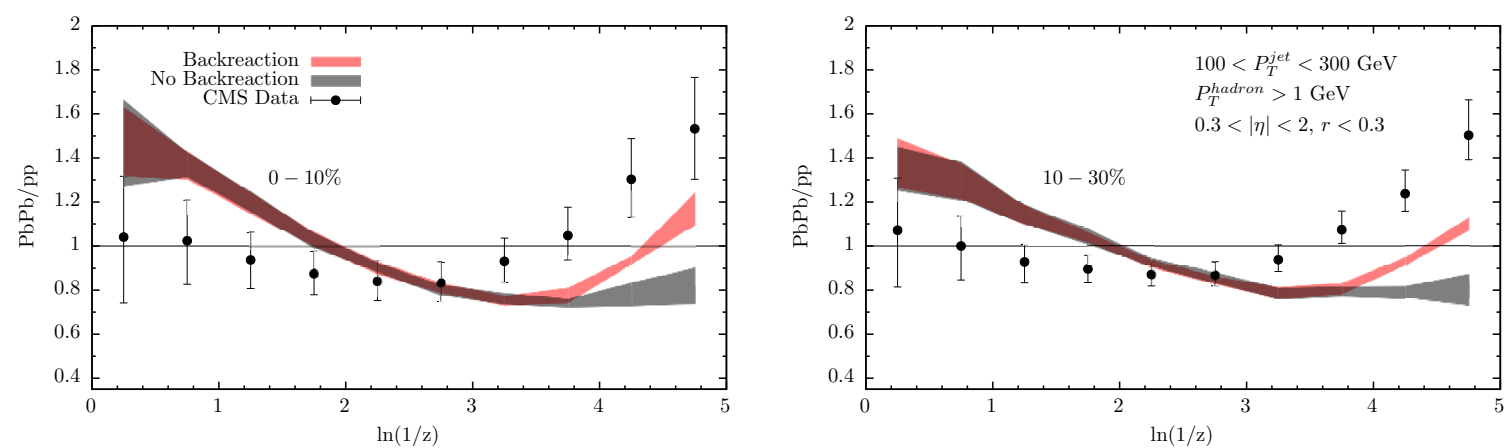

Figure 11. Ratio of the jet fragmentation function in $\mathrm{PbPb}$ collisions at $\sqrt{s}=2.76 \mathrm{ATeV}$ to that in proton-proton collisions. As in figure 10, we compare the results of our hybrid model calculation with and without the inclusion of the particles coming from the backreaction of the medium in 0-10\% centrality (left) and 10-30\% centrality (right) collisions to data measured by CMS [51].

By comparing the results of our simulations with and without backreaction, the jet fragmentation functions clearly show where the particles resulting from the wake in the plasma that get reconstructed as part of the jet end up. The hard part of the jet is practically unaffected by the backreaction of the medium, with an almost identical distribution of hard fragments in the two calculations. (The small differences arise from the the small change in reconstructed jet momentum associated with the addition of soft particles from the backreaction of the medium to the jet.) Both of the simulations show an enhancement of hard fragments at the largest values of $z$ (smallest $\log 1 / z$ ) in $\mathrm{PbPb}$ collisions. As we have seen in other ways, wide jets (with more softer fragments) lose more energy, so at any given energy the jets that remain tend to be narrower, and tend to contain fewer, and therefore more energetic fragments, than in proton-proton collisions. Such an enhancement therefore seems generic to any mechanism of energy loss which significantly reduces the soft, large angle, components of jets. (See ref. [105] for a similar effect in a perturbative-based jet quenching Monte Carlo [76, 77, 81, 82].) The small- $z$ region of the fragmentation function is sensitive to backreaction effects. The emission of soft particles by the jet-induced wake compensates the suppression of soft fragments due to energy loss and leads to an overall enhancement of soft tracks in the $\mathrm{PbPb}$ jets relative to proton-proton jets. The comparison between our calculations with and without the particles coming from the backreaction of the medium also shows the range of momenta at which back reaction contributes significantly to the particles reconstructed as part of a jet, namely $p_{\mathrm{T}}$ up to $p_{\mathrm{T}} \sim 2.5 \mathrm{GeV}$. At this scale our approximate approach to the wake distribution eq. (4.7) underestimates particle production, as we discussed after eq. (4.5). At softer momenta, by neglecting the effects of viscosity as the sound waves produced by the jet damp out and heat the plasma we are underestimating the particle production also.

Comparing the fragmentation function ratios that we have obtained in our calculations including the particles from the backreaction of the medium to the ratios measured by CMS [51], we see qualitative similarities, but not quantitative agreement. At large $z$ (small $\ln (1 / z))$ our calculated ratio is above 1 while there is no evidence for this in the data. 
However, in this regime the uncertainties in both data and theory are significant. At small $z$ (large $\ln (1 / z)$ ) we see that the particles from the wake in the plasma that are reconstructed as part of the jet turn the fragmentation function ratio upwards, but they do not turn it as far upward as in the data, and they turn it upward at a larger value of $\ln (1 / z)$ than in the data. This suggests that our treatment of the back reaction of the medium is missing an increase in the production of few $\mathrm{GeV}$ particles. We have already suggested several possible interpretations of this above. It is quite reasonable to expect that whatever physics it is that our approximations have missed that would push the large $\ln (1 / z)$ fragmentation function ratios up would also push the jet shapes up at larger values of $r$ in figure 10. We are missing some soft to few $\mathrm{GeV}$ particles at these values of $r$.

Certainly a more complete description of the backreaction dynamics, from the wake in the plasma to the resulting particles reconstructed as part of a jet, would be helpful. It is also worth mentioning, though, that we have found a disagreement between the fragmentation functions from PYTHIA simulations of jets in proton-proton collisions and the fragmentation functions measured in small $R$ jets in these collisions, for example with the fragmentation functions from РҮтнIA high by as much as $30 \%$ at small $z$, at least with the Pythia tunes we have explored (Monash, $4 \mathrm{C}$ and $4 \mathrm{C}$ with a modified $Q_{0}$ parameter). A similar disagreement has also been found between state-of-the-art parametrizations of fragmentation functions and proton-proton data [186]. These uncertainties are comparable in size to the size of medium modifications themselves. This means that understanding fragmentation function ratios more quantitatively will require a better understanding of hadronization of jets, both those in vacuum and those that have been modified by passage through a medium. Regardless, it is already an inescapable conclusion that backreaction dynamics has a significant impact on the jet fragmentation function at small $z$.

In the next section we will explore a suite of observables that are even more sensitive to the soft particles from the wake in the plasma that end up reconstructed as a part of the jet than the jet shape or the jet fragmentation function.

\subsection{Recovering the "lost" jet energy and "missing" $p_{\mathrm{T}}$ deposited by a di- jet pair}

We have seen in the previous subsection that the backreaction of the medium in response to the passage of a jet, namely the wake in the plasma, contributes to single jet observables like the jet $R_{\mathrm{AA}}$, jet shape, and jet fragmentation function because some of the particles that result from the hadronization of the wake in the plasma must of necessity end up counted as part of the jet after the jet is reconstructed and the background is subtracted. In these observables, the effects of the particles coming from hadronizing the wake in the plasma constitute small corrections overall, although they can play a significant role in certain kinematic regimes (like in the soft region of the fragmentation function ratio and the large $r$ region of the jet shape ratio). In this subsection, we focus on a suite of observables which are dominated by effects originating from the backreaction of the medium in response to the passage of two jets, a dijet, through it.

The principles behind our simplified calculation of the spectrum of particles produced as the medium (including the wake therein) hadronizes are that the medium acquires 
the energy and momentum lost by the jets passing through it, which thermalizes subject to energy and momentum conservation. This implies collective motion of the medium, a wake in the quark-gluon plasma. After hadronization, this energy and momentum is recovered in the form of soft particles with a wide angular distribution given by eq. (4.7). This makes it natural for us to calculate the so-called "missing- $p_{\mathrm{T}}$ observables" introduced recently by CMS [60], and compare our calculations to measurements made by CMS. These observables are defined to extract the spectrum and angular distribution of the particles that correspond to the net momentum lost by the two jets in a dijet pair. Each jet in the pair loses energy and momentum to the plasma, but in general one will lose more than the other. By constructing observables designed to recover the net momentum lost by the pair of jets we obtain observables that are dominated by the response of the medium to the jets. These observables are constructed from all the tracks in an event, not just those reconstructed as part of a jet. We shall set $K=0$ throughout this subsection, neglecting transverse momentum broadening. We have obtained results with $K \neq 0$, but the $K$-dependent effects that we see in these missing- $p_{\mathrm{T}}$ observables are all much smaller in magnitude than the effects of particles coming from the hadronization of the wake in the plasma. As these observable consequences of the backreaction of the medium are our focus in this subsection, there is no reason to introduce $K \neq 0$.

The observables that we look at in this subsection are intrinsically hadronic and, therefore, are hard to bring under full theoretical control without significant modelling of the differences between hadronization in heavy ion collisions and proton-proton collisions. Nevertheless, we will observe several qualitative features of our results which are similar to what is seen in the experimental data. This indicates that our treatment, although simplified, captures some of the main aspects of the collective reaction of the plasma to the deposited jet energy.

Following ref. [60], the missing $p_{\mathrm{T}}$ analysis consists in studying the conservation of momentum in heavy ion events which include hard jets reconstructed with a specified anti$k_{t}$ reconstruction parameter $R$. The first step in the analysis is to use the anti- $k_{t}$ algorithm to find a sample of events containing at least two jets and to determine the $p_{\mathrm{T}}$ and the jet axis of the leading and subleading jet in each event. We then define the dijet angle $\phi_{\text {dijet }}$ as the bisection between $\phi_{\text {leading }}$ and $-\phi_{\text {subleading, }}$, where $\phi_{(\text {sub }) \text { leading }}$ is the azimuthal angle of the (sub)leading jet. The only use of the jet reconstruction algorithm is the selection of the event sample and the determination of the jet $p_{\mathrm{T}}$ 's and axes and hence $\phi_{\text {dijet }}$. In the remainder of the analysis, all charged tracks in the events in the sample are used.

The next step in the analysis is to project the momenta of each of the charged tracks in an event along the dijet axis. This projection is defined by

$$
\not p_{\mathrm{T}}^{\|} \equiv-p_{\mathrm{T}} \cos \left(\phi_{\text {dijet }}-\phi\right)
$$

where $p_{\mathrm{T}}$ and $\phi$ are the transverse momentum and azimuthal angle of the track. With this convention, $\not_{\mathrm{T}}^{\|}$is positive for tracks in the subleading jet hemisphere and negative for tracks in the leading jet hemisphere. By momentum conservation, the sum of all the $\not p_{\mathrm{T}}^{\|}$for all the tracks in an event must be zero. However, in the experimental analysis only charged 
tracks with $|\eta|<3$ are considered, and therefore, the net $\not p_{\mathrm{T}}^{\|}$need not cancel identically. Nevertheless, by studying the approximate cancellation of this momentum component as a function of which tracks we include in the analysis, out to what angular distance from the dijet axis, we can extract valuable information about the distribution of the lost jet energy.

We start by computing the $\not p_{\mathrm{T}}^{\|}$distribution sliced in $\Delta$ bins, where $\Delta$ is the distance in the $(\eta, \phi)$ plane between the track in question and either the leading jet axis or the subleading jet axis, whichever the track is closest to. We consider dijet pairs with leading and subleading transverse momenta $p_{\mathrm{T}}^{\text {leading }}>120 \mathrm{GeV}$ and $p_{\mathrm{T}}{ }^{\text {subleading }}>50 \mathrm{GeV}$ respectively, and with both jets within $|\eta|<2$. We also limit our sample to events in which the two jets are back-to-back, our criterion being $\Delta \phi>5 \pi / 6$. Finally, after identifying the dijet pair, only those dijets in which both jets have $|\eta|<0.6$ are considered. (We start by finding jets within a much larger $|\eta|$ window in order to be as confident as we can be that when we have identified a dijet in which both jets have $|\eta|<0.6$ these two jets really are the leading and subleading jets in the event.) All these specifications of our event sample are the same as those in the experimental analysis of ref. [60]. The result of this analysis is shown in figure 12 for our full calculations including the medium response (left) and, for comparison, without medium backreaction (right). In the right panel, we confirm that momentum is not conserved in our hybrid model by its construction, when we do not include the particles coming from the wake in the plasma. The left-most bin in $\Delta$ shows the momentum imbalance between the high-momentum tracks in the two dijets themselves, with more momentum in the leading jet direction and less in the subleading jet direction. In our hybrid model the two jets have lost different amounts of momentum, and in the right panel we have neglected the fact that the lost momentum is deposited as two wakes in the medium. The left panel of figure 12 clearly shows how the inclusion of the response of the medium, modeled via eq. (4.7), results in a population of soft particles spread broadly in $\Delta$ with a net momentum in the subleading jet hemisphere (positive $\not p_{\mathrm{T}}^{\|}$). The subleading jet has lost more momentum, and hence the wake in the plasma moving in the subleading jet direction contains more momentum than the wake moving in the leading jet direction.

Another way to see the effect of including the backreaction of the medium is to compare the solid black curves in the two panels of figure 12 . While the accumulated $\not p_{\mathrm{T}}^{\|}$tends to zero as $\Delta$ increases in the calculations in the left panel, in the right panel the accumulated $\not p_{\mathrm{T}}^{\|}$is $\sim 20 \mathrm{GeV}$ after all $\Delta$ bins have been summed. This value, which corresponds to the average imbalance in the energy lost by the two jets in the dijet pair, compares well with simple estimates [85]. The medium response transforms the "lost" energy from the two jets into two wakes, and hence into softer particles in the range of $\sim 0.5-2 \mathrm{GeV}$ whose net momentum is preferentially in the hemisphere of the more quenched, subleading, jet. This is shown by the enhancement of soft $\not p_{\mathrm{T}}^{\|}$tracks observed in the left panel of the figure.

The dashed curve, which is the same in both panels, shows the accumulated $\not p_{\mathrm{T}}^{\|}$for proton-proton collision events as described by our PYTHIA tune. Recalling that our analysis employs all the tracks in an event, not just those reconstructed as part of a jet, it is perhaps not too surprising that the $\not \|_{\mathrm{T}}^{\|}$distribution generated by РҮтнIA that is described by the 

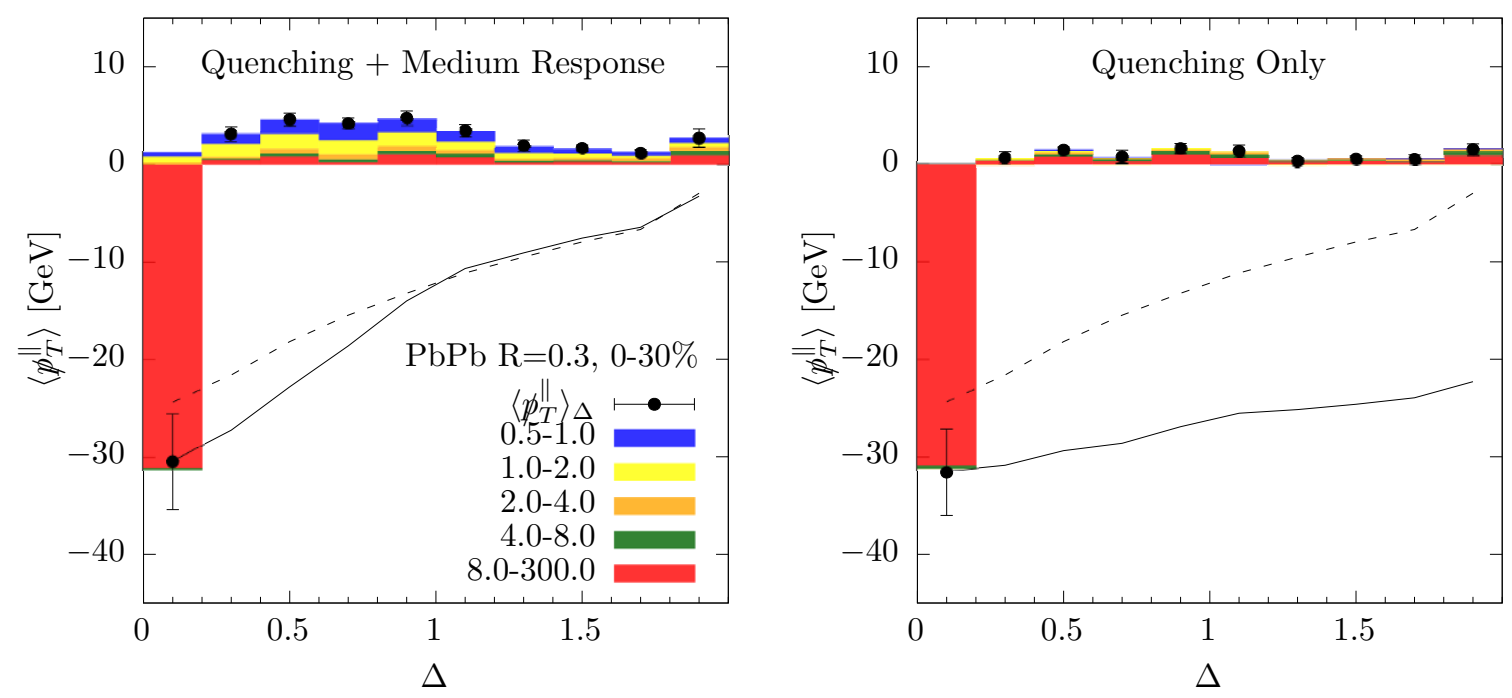

Figure 12. Results from our calculations of the missing $\not p_{\mathrm{T}}^{\|}$for tracks that are $\Delta$ away from the dijet axis in $(\eta, \phi)$-space, in $\mathrm{PbPb}$ collisions containing a pair of $R=0.3$ jets satisfying kinematic criteria described in the text. The first nine bins in $\Delta$ are each 0.2 wide, together covering $0<\Delta<1.8$. The tenth bin, plotted at $\Delta=1.9$, contains the missing $\not p_{\mathrm{T}}^{\|}$for all the tracks with $\Delta>1.8$. Note that we limit our analysis to tracks with $|\eta|<3$. In the left panel, we show the results of our full calculation, with energy loss calculated within our hybrid model with no momentum broadening, and with particles coming from the backreaction of the medium included. The right panel shows our results if we leave out the particles coming from the backreaction of the medium. In both plots, the solid black points show the net $\not p_{\mathrm{T}}^{\|}$of all charged tracks with $|\eta|<3$ in each $\Delta$ bin. The contribution to this net momentum of tracks in different momentum bins are codified by the colored histograms. In both plots, the solid line shows the cumulative sum of all the $\not p_{\mathrm{T}}^{\|}$in a $\Delta$ bin and all the $\Delta$ bins to its left. The black points, the colored histograms, and the solid line are all calculated for $0-30 \%$ central $\mathrm{PbPb}$ collisions with $\sqrt{s}=2.76 \mathrm{ATeV}$. For comparison, we have repeated the calculation for proton-proton collisions as described by our PYTHIA tune, and the dashed line in each plot shows the same cumulative sum of $\not p_{\mathrm{T}}^{\|}$for proton-proton collisions that the solid line shows for $\mathrm{PbPb}$ collisions.

dashed curve is not in full agreement with measurements made in proton-proton collisions reported in ref. [60]. This small discrepancy illustrates the difficulty in obtaining full theoretical control over this inherently hadronic observable. What we shall do in subsequent figures is to subtract the $\not \|_{\mathrm{T}}^{\|}$distribution in our PүтнIA calculation of proton-proton calculations from the $\not p_{\mathrm{T}}^{\|}$distribution in our $\mathrm{PbPb}$ calculation including black-reaction. That is, we shall subtract the dashed curve from the solid curve in the left panel of figure 12. In this way, we shall focus explicitly on effects that are due to medium-induced energy loss, and the wake in the medium, both of which our model is intended to describe.

By comparing the solid and dashed curves in the left panel of figure 12, we see that the net momentum lost by the pair of jets is distributed over a wide angular region, over $\Delta \lesssim 1$. We can also see from the similarity between the red histograms in the two panels in the figure that the backreaction of the medium does not affect the hard tracks in the event, namely the hard components of the jet. We also see that almost the entire imbalance in the 
high-momentum tracks is an imbalance at small $\Delta$ with the net momentum in the leading jet hemisphere, whereas almost the entire imbalance in soft tracks is in the hemisphere of the event in which that corresponds to particles from the (larger) wake of the subleading jet. The experimentally measured distributions [60] are quite similar to what we see in the left panel of figure 12, but because of the discrepancy between our calculations and data for proton-proton collisions we will postpone a quantitative comparison until we have results for the difference between the missing momentum in $\mathrm{PbPb}$ and proton-proton collisions, below.

Following ref. [60], we sharpen the analysis by studying how the $p_{\mathrm{T}}^{\|}$distribution depends on the dijet asymmetry $A_{J} \equiv\left(p_{\mathrm{T}}^{\text {leading }}-p_{\mathrm{T}}^{\text {subleading }}\right) /\left(p_{\mathrm{T}}^{\text {leading }}+p_{\mathrm{T}}{ }^{\text {subleading }}\right)$. In events in which the dijet pair features a larger $A_{J}$ we should expect a correspondingly larger momentum asymmetry in the soft particles coming from the hadronization of the wakes of the two jets. As discussed above and as in ref. [60], we shall also focus on the difference between the $\not p_{\mathrm{T}}^{\|}$distribution in $\mathrm{PbPb}$ collisions and that in proton-proton collisions containing dijets satisfying the same kinematic criteria. Jet quenching makes the $A_{J}$-distribution wider in $\mathrm{PbPb}$ collisions, but it is already rather wide in proton-proton collisions because dijets are often produced in association with a third jet or other radiation, making it important for us to focus on the difference between $\mathrm{PbPb}$ and proton-proton collisions.

In the upper and middle panels of figure 13 we show the value of $p p_{\mathrm{T}}^{\|}$obtained from our calculations upon integrating over all angular separations $\Delta$ in $\mathrm{PbPb}$ collisions minus the same obtained in proton-proton collisions, in both cases for dijet events reconstructed using the anti- $k_{t}$ algorithm with $R=0.3$ in bins of the dijet asymmetry $A_{J}$, in both cases for collisions with two different centralities. In the upper panels, we include the modifications to the jets because of energy loss but leave out the contributions from the backreaction of the medium. The middle panels show the results from our full calculation, including both the modifications to the jets and the wakes in the plasma. The black points in the upper panels show the momentum imbalance in dijet events when the momentum carried by the wakes are neglected. The black points in the middle panels confirm that total momentum is conserved regardless of the asymmetry $A_{J}$ - once we include the particles coming from the wakes in the plasma. We see from the red, green and orange histograms in the upper panels of figure 13, though, that events with a larger and larger dijet asymmetry $A_{J}$ feature more and more missing $\not p_{\mathrm{T}}^{\|}$, in particular for tracks with $p_{\mathrm{T}}>2 \mathrm{GeV}$, with a sign corresponding to there being more tracks with these (hard) momenta in the leading jet direction. That is, jet quenching - here described in our hybrid model — suppresses the hard particle contribution in the subleading jet hemisphere, as the subleading jet has lost more energy. The energy and momenta lost by both jets have been thermalized as wakes, which in turn are transformed into softer hadrons when the medium including the wakes freezes out. We see the contributions of the momentum carried by hadrons coming from the wakes in the plasma by comparing the upper and middle panels of figure 13. In the blue and yellow histograms in the upper panels of the figure, we see the missing $\not p_{\mathrm{T}}^{\|}$ for tracks with $p_{\mathrm{T}}<2 \mathrm{GeV}$ with a sign corresponding to there being more tracks with these (soft) momenta in the subleading jet direction. Since the subleading jet lost more momentum, its wake contains more momentum and this shows up in the missing $\not p_{\mathrm{T}}^{\|}$of the 

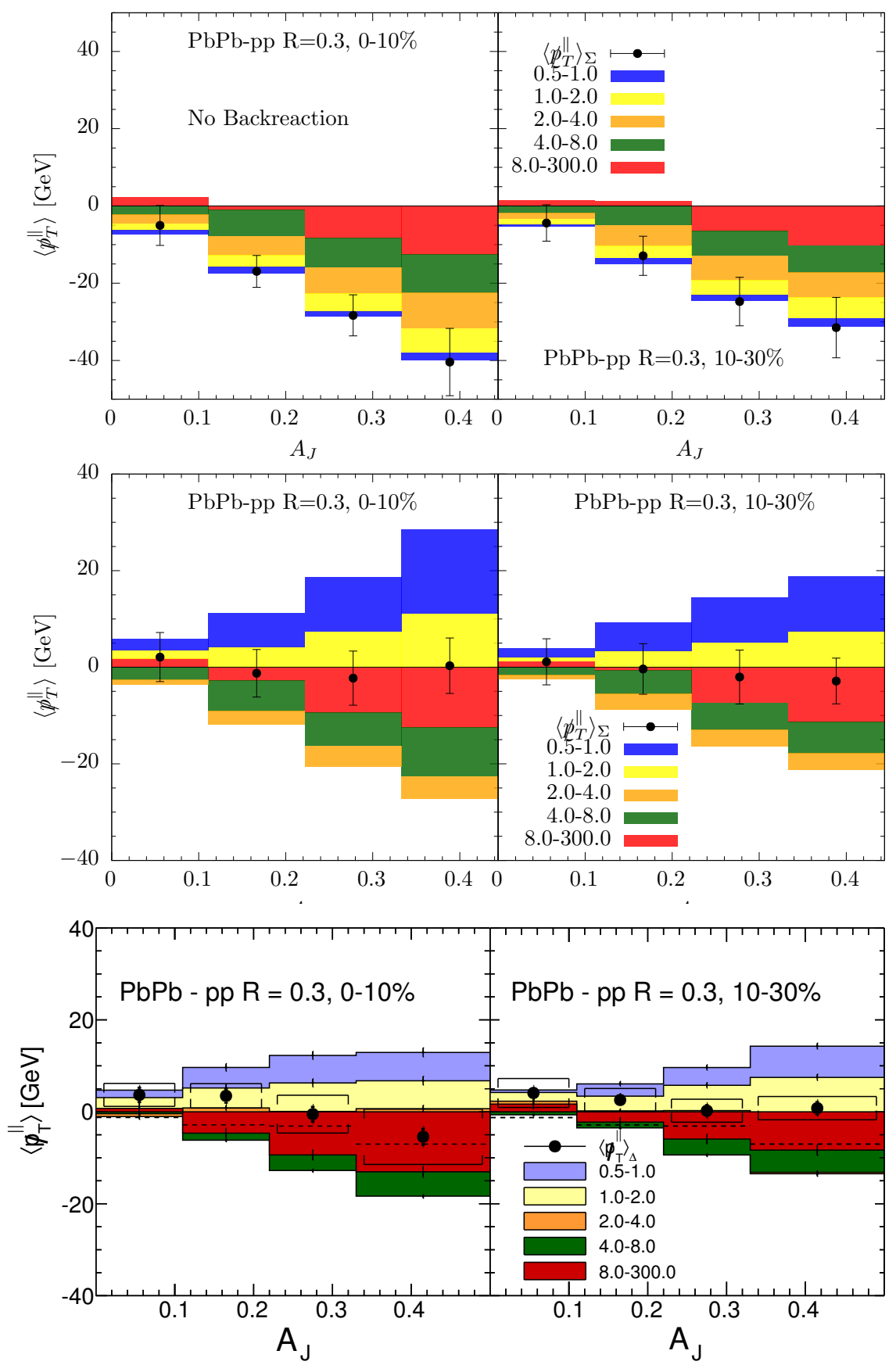

Figure 13. The middle two panels show the results of our full calculation, while the upper two panels show the results from our hybrid model for energy loss with the contribution from the backreaction of the medium left out. Black points with error bars show the difference between the missing $\not p_{\mathrm{T}}^{\|}$in $\mathrm{PbPb}$ and pp collisions, integrated over all $\Delta$, summed over all track momenta, binned in the dijet asymmetry $A_{J}$, for events containing $R=0.3$ dijets in collisions with $0-10 \%$ (left) and $10-30 \%$ (right) centrality. The colored histograms show the missing $\not_{\mathrm{T}}^{\|}$for tracks whose momenta is in the indicated range. The lower two panels, obtained from ref. [60], show the results from the same analysis performed on experimental data by the CMS collaboration. 
soft tracks in the event. All these effects are larger in the central collisions on the left than in the more peripheral collisions on the right, as expected since due to the smaller size of the fireball the dijets are on average less quenched in more peripheral collisions.

Almost all the features seen in the middle panels of figure 13 that we have described above are present in the missing $\not p_{\mathrm{T}}^{\|}$measurements reported by the CMS collaboration [60] that we show as the lower panels in figure 13, at the least at a qualitative level and in most cases to a degree that constitutes agreement within the uncertainties.

The biggest difference between the results of our calculations and the experimental results is found for semi-hard particles in the $2 \mathrm{GeV}<p_{\mathrm{T}}<4 \mathrm{GeV}$ momentum range. By comparing the orange histograms in the upper and middle panels of figure 13, we see that in our calculation the wake contributes little in this momentum range: the "orange contribution" to the missing $\not p_{\mathrm{T}}^{\|}$imbalance due to jet quenching that is seen in the upper panels remains almost unmodified in the middle panels. Instead, in the data there is almost no missing $\not p_{\mathrm{T}}^{\|}$imbalance in this momentum range. Furthermore, by comparing the upper and middle panels we see that in our calculation the contribution to the missing $\not p_{\mathrm{T}}^{\|}$ from soft particles with $p_{T}<2 \mathrm{GeV}$ is dominated by the effects of the backreaction of the medium, and then by comparing the middle and lower panels we see that our calculations yield a greater contribution from these soft particles than in the data. So, although our implementation of medium backreaction does restore momentum conservation - the $\not p_{\mathrm{T}}^{\|}$ excess in hard particles in the direction of the leading jet due to jet quenching is balanced by the $\not p_{\mathrm{T}}^{\|}$excess in soft particles in the direction of the subleading jet coming from the wake in the plasma - our calculation of the particles coming from the wake seems to yield somewhat more particles with $p_{\mathrm{T}}<2 \mathrm{GeV}$ than in the data and substantially fewer particles with $2 \mathrm{GeV}<p_{\mathrm{T}}<4 \mathrm{GeV}$. We will defer further discussion of the origin of this discrepancy to section 5. For the present it suffices to remember from our discussion earlier in this section that several of the approximations that we used in setting up our crude treatment of the particles from the wake break down for particles with $p_{\mathrm{T}}$ substantially greater than the temperature of the medium at freezeout, and they break down in a way such that we anticipated underpredicting the production of semi-hard particles from the wake.

Finally, we turn to the dependence of the missing $\not p_{T}^{\|}$distribution on the value of the parameter $R$ used in finding and reconstructing the jets, recalling that using a large $R$ results in finding a sample of jets that have a larger average angular size. In figure 14 we show the $\not p_{\mathrm{T}}^{\|}$distribution as a function of the angular separation $\Delta$ for different values of the jet reconstruction parameter. For the purposes of focusing on the effects of jet quenching and in order to make a comparison with experimental data, we show the difference between $\mathrm{PbPb}$ and proton-proton collisions. As in the previous plots, the colored histograms show the contributions of tracks in different $p_{\mathrm{T}}$-ranges, and the black points show $\not_{\mathrm{T}}^{\|}$for tracks with all values of $p_{T}$ in a given $\Delta$-bin. (As always when we show results from our calculations, the error bars include the uncertainties coming from the error bar on the experimental data point that we used to fix the $\kappa_{\mathrm{sc}}$ parameter in our hybrid model and from our estimate of the systematic uncertainty in our model that we make by varying the temperature $T_{c}$ below which we turn off parton energy loss.) As in figure 12, in the middle 

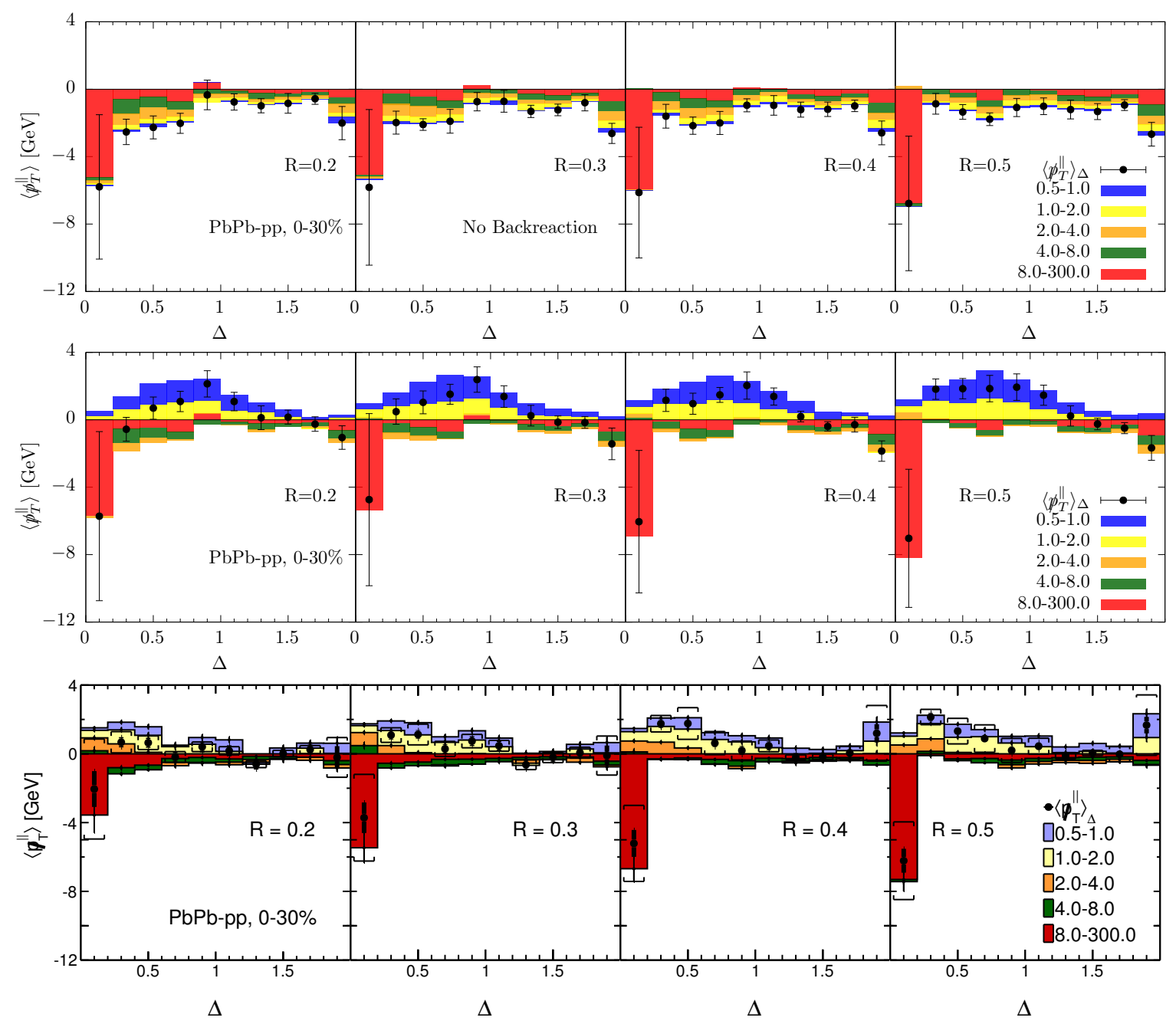

Figure 14. Evolution in $R$ of the missing $\not p_{\mathrm{T}}^{\|}$observable, $A_{J}$ inclusive and sliced in $\Delta$, for the difference between $\mathrm{PbPb}$ and pp collisions. Top four panels are the results of our calculations with no contributions from the backreaction of the medium. Middle four panels are the results of our full calculations, in which the $p_{\mathrm{T}}^{\|}$imbalance in hard particles due to jet quenching is balanced by that in soft particles coming from the wakes of the two jets. The $R=0.3$ panels are the difference between the $\mathrm{PbPb}$ results shown in figure 12 and the same for proton-proton collisions. Bottom four panels come from the analysis of experimental data by the CMS collaboration reported in ref. [60]. There is some hint of $R$-dependence, despite the big error bars, especially in the first $\Delta$ bin.

panels of figure 14 momentum conservation is (approximately) recovered after summing over all $\Delta$ bins, although not precisely because we only use tracks with $p_{\mathrm{T}}>0.5 \mathrm{GeV}$ and $|\eta|<3$ in the analysis.

As already mentioned, in this analysis the anti- $k_{t}$ reconstruction algorithm is only employed to determine the sample of events over which the $\not p_{\mathrm{T}}^{\|}$distribution is computed, as well as the jet and dijet axes in these events. All tracks in the events are then used in the analysis, whether or not the reconstruction algorithm counted them as being within a jet. By increasing the anti- $k_{t}$ parameter $R$ while keeping the momentum cuts on the dijet 
selection fixed, we include jets that are on average wider in angle and that include more, and hence softer, fragments. Since wider jets containing a larger number of fragments lose more energy, the missing $\not p_{\mathrm{T}}^{\|}$in the hardest $p_{T}$ tracks in the smallest $\Delta$ bin grows in magnitude with increasing $R$. Despite the large error bars in our calculations and in data, this enhancement is present in both, as seen in figure 14. This is further evidence for the fact that wider jets lose more energy than narrower jets do.

Beyond the first $\Delta$ bin, we see in figure 14 that the distribution of $\not p_{T}^{\|}$is dominated by softer particles with net $\not p_{\mathrm{T}}^{\|}$in the subleading jet direction. The $\Delta$-dependence of the softest components of the $\not p_{\mathrm{T}}^{\|}$distribution shows very little sensitivity to $R$. This may be understood as a consequence of the wide angular region in which energy is recovered, controlled by eq. (4.7). If we look carefully at the $p_{\mathrm{T}}^{\|}$distribution for the hardest particles in the $0.2<\Delta<0.8$ range of angles, we do see an $R$-dependence in the results from our calculations and in data: the $\not p_{T}^{\|}$imbalance in this kinematic regime decreases with increasing $R$. In our calculations and in the data, this imbalance comes from the protonproton collisions: there is no significant imbalance in the hard particles at these angles in the $\mathrm{PbPb}$ collisions for any $R$. This indicates that the effect is a sign of the presence of third jets at these angles. When $R$ is small, a structure that would have been reconstructed as a single jet if $R$ were larger can be reconstructed instead as two nearby jets, which end up being counted as the subleading jet in a dijet and as a third jet. The third jet results in an imbalance in the $\not p_{\mathrm{T}}^{\|}$distribution for hard particles away from the dijet axis in the protonproton collisions, but this weak third jet is greatly quenched in the $\mathrm{PbPb}$ collisions. The result in the difference between $\mathrm{PbPb}$ and proton-proton collisions is as seen in figure 14, and the effect is reduced when jets are reconstructed with larger $R$.

Remarkably, for all the features in figure 14 that we have described to this point there is good qualitative agreement between the results of our calculations in the middle panels and the experimental distributions reported in [60] that we show in the lower panels. We do wish to note two qualitative disagreements, however. First, our results for the missing $\not p_{\mathrm{T}}^{\|}$distribution differ from the experimental results in the last bin in $\Delta$ which, we recall, corresponds to the $\not p_{\mathrm{T}}^{\|}$imbalance for particles with all $\Delta>1.8$. We have checked that this disagreement is entirely due to a difference between our results and experimental results for proton-proton collisions; we do not know how to interpret this particular difference between Pythia and experiment. Second, as in figure 13 we see that our calculation of the $\not p_{T}^{\|}$distribution of the particles coming from the backreaction of the plasma to the jets, (which we can discern by comparing the middle panels to the top panels) is lacking in "orange particles" with $2 \mathrm{GeV}<p_{\mathrm{T}}<4 \mathrm{GeV}$ relative to what we see in the data in the lower panels. Correspondingly, our calculation restores momentum conservation with a greater imbalance in the softest particles with $p_{\mathrm{T}}<2 \mathrm{GeV}$ than in the data. Our calculation of the particles coming from the wakes in the plasma has significantly fewer semi-hard particles and somewhat more soft particles. We will discuss this disagreement further in the next section.

Before we move on, it is important to look back at the way in which we defined the new observable that we introduced at the end of section 3 that, as we argued in our discussion 
around figure 6 and figure 7 , is particularly sensitive to the effects of transverse momentum broadening. In constructing the hadronic observable in figure 7 , we only used particles with $5 \mathrm{GeV}<p_{\mathrm{T}}<10 \mathrm{GeV}$. We can now see clearly why we chose not to use particles with $p_{\mathrm{T}}<5 \mathrm{GeV}$ in this observable. We want this observable to only be sensitive to (modifications of) the jets themselves. We want it to be unaffected by the wake in the plasma and, more generally, by the energy lost from the jet wherever that lost energy ends up. In our calculation, that lost energy dominates the missing $\not p_{\mathrm{T}}^{\|}$distribution for $p_{\mathrm{T}}<2 \mathrm{GeV}$; in the data, it is clear that it is important for $p_{\mathrm{T}}<4 \mathrm{GeV}$. In order to avoid all the physics that is of interest to us here in section 4, in figure 7 in section 3 we ignored particles with $p_{\mathrm{T}}<5 \mathrm{GeV}$.

\section{Discussion and outlook}

In this paper we have described the origin and observable consequences of two important effects upon implementing and describing them in our hybrid model of jet quenching: transverse momentum broadening, as the partons in the jet are kicked transverse to their direction of motion during their passage through the medium, and the backreaction of the medium to the momentum and energy deposited in it by the passage of the jet through it.

Our discussion of transverse momentum broadening is based on a Gaussian distribution of kicks in transverse momentum experienced by each parton in the jet. This approximation captures the bulk of the distribution of transverse momentum-transfer but neglects rare scatterings by larger angles that impart more transverse momentum. Quite surprisingly, we have found that adding Gaussian momentum broadening has little impact on typical jet observables such as the jet spectrum, jet shapes and dijet acoplanarity, even when we choose unrealistically large values of $K \equiv \hat{q} / T^{3}$, the new second parameter that we add to our model to quantify the strength of the transverse momentum broadening. The reason why it is hard to see effects of broadening in these observables can be traced back to the parton energy loss and consequent jet quenching, described successfully by our hybrid model. We find that in our hybrid model parton energy loss results in jets with a given energy observed in $\mathrm{PbPb}$ collisions being narrower than jets with the same energy in proton-proton collisions. This happens because jets that are wider in opening angle lose more energy than those that are narrower and because the jet energy spectrum is steeply falling, and has also been seen recently in entirely weakly coupled analyses [105] and in entirely strongly coupled analyses [147]. This narrowing of jets with a given energy because of energy loss turns out to be a larger effect than the broadening of jets due to kicks to the transverse momentum of partons in the jets. Also, for narrower jets the principal effect of transverse kicks is a change in the overall direction of the jet, rather than changes to its shape. (As an extreme case, think of a jet containing only one parton.) For a high energy jet the resulting acoplanarity is small, but for narrow jets the change to their shape is even smaller. (In the extreme case of a single parton, the shape of the resulting jet does not change at all if the direction of that parton changes.) Although initially surprising, once understood these considerations make it clear that it will be exceedingly difficult to extract the value of $K$, or the jet quenching parameter $\hat{q}$, directly from the effect of transverse momentum 
broadening on traditional jet observables. (In weakly coupled analyses but not in general, $\hat{q}$ is related to parton energy loss and so can be constrained indirectly via observables that are sensitive to energy loss.)

We have, however, found a new kind of jet shape ratio that is partially differential in the $p_{\mathrm{T}}$ of the tracks entering its definition that does exhibit sensitivity to the value of $K$. In figure 6 we restrict the $p_{\mathrm{T}}$-range of the partons entering the analysis to be $10<p_{\mathrm{T}}{ }^{\text {parton }}<20 \mathrm{GeV}$, as among the options we have investigated it is for partons in this momentum range that we have found the most sensitivity to the value of $K$. We then need a hadronic observable that is sensitive to the angular distribution of partons in this momentum range, and in figure 7 we construct a partially differential jet shape ratio observable using hadrons in the $p_{\mathrm{T}}$-range $5<p_{\mathrm{T}}^{\text {hadron }}<10 \mathrm{GeV}$. The results we obtain indicate that experimental measurements of this observable, or similar observables, could be used to constrain the value of the broadening parameter $K$, a key property of the medium. Restricting attention to $p_{\mathrm{T}}$ above a minimum value (like the $5 \mathrm{GeV}$ we have chosen) is important because it makes the observable insensitive to the other physical effect that we describe in this paper, namely the reaction of the medium to the passage of the jet. More on that below. Restricting attention to $p_{\mathrm{T}}$ below a maximum value (like the $10 \mathrm{GeV}$ we have chosen) ensures that we are looking at jets containing many tracks and are looking at tracks that are not too energetic and thus can be affected visibly by transverse momentum broadening.

Although the successes of our model to date [148, 149] motivate the investments in improving it that we describe in this paper, in the long run the most instructive use of a simple few-parameter model like our hybrid model is to discover and understand the ways in which it fails to describe data. Let us look ahead by imagining one particularly interesting way in which this could happen in future. Let us imagine a day when the measurement of observables like the partially differential jet shape ratio $\Psi_{p_{\mathrm{T}}}^{\text {subleading }}(r)$ plotted in figure 7 have been used to discover experimental evidence for medium-induced transverse momentum broadening and to constrain the value of $K$. What will the next step be? At such a point in time, the key next question will be to ask whether the event-by-event and parton-by-parton distribution of the transverse kicks contributing to an ensemble-averaged observable like $\Psi_{p_{\mathrm{T}}}^{\text {subleading }}(r)$ is indeed Gaussian. This distribution is Gaussian by hypothesis in our hybrid model, meaning that our model will ultimately serve as a baseline against which to look for evidence of a non-Gaussian distribution. If the quark-gluon plasma in QCD were strongly coupled on all length scales, including short length scales, the distribution of transverse kicks would be Gaussian for all values of the momentum transfer, including large momentum transfers. This is not what is expected in an asymptotically free theory like QCD. If the strongly coupled liquid QCD plasma can be probed at high enough momentum transfer, the weakly coupled quarks and gluons present within it at short length scales should manifest themselves. Once the overall strength of the broadening $K$ has been constrained, the way to look for point-like scatterers within the strongly coupled plasma will be to look for rare events in which a parton within a jet is scattered by an angle that would be improbably large if the distribution of transverse momentum kicks were a Gaussian with overall strength specified by $K[151,170]$. The hallmark of 
point-like scatterers is a distribution of momentum transfer that has a power-law tail at large momentum transfers. If in future our hybrid model, with its Gaussian distribution of transverse kicks with strength $K$, fails to describe data because it neglects rare (but only power-law-rare) large angle scatterings, their discovery may potentially be used to determine at which small length scale the fluctuations within strongly coupled quark-gluon plasma behave as weakly coupled quark and gluon quasiparticles.

We find that standard intrajet observables, like the conventional jet shape and jet fragmentation function, are sensitive to the presence of the wake in the plasma left behind by the passing jet. Since the wake carries the momentum lost by the jet, the particles in the final state that come from the hadronization of the medium including the wake must have net momentum in the direction of the jet, meaning that some component of what experimentalists reconstruct as a jet, even after background subtraction, must in fact be composed of soft particles coming from the wake in the plasma. Motivated by expectations coming from explicit calculations of the wake in strongly coupled plasma $[123,125]$ and by recent weakly coupled calculations in which the energy lost by a jet cascades into multiple soft particles [150-156] we analyze the wake upon making the simplifying assumptions that all the energy and momentum lost by the jet is incorporated into hydrodynamic motion and that the resulting perturbations to the temperature and velocity of the hydrodynamic fluid are small. That is, we assume that all of the lost momentum and energy go into a hydrodynamic wake that is as thermalized as it can be by the time of freezeout, yielding only small corrections to the spectra of the hadrons in the final state, a final state that remembers nothing about the origin of this perturbation other than the momentum and energy dumped into the plasma. (In a weakly coupled calculation as in [156] in which a part of the lost energy hydrodynamizes, these assumptions are relevant for this component of the lost energy, denoted $\Delta E_{\text {thermal }}$ in [156].) In our calculation of the corrections to the hadronic spectra coming from the wake in the plasma, we have made further simplifying assumptions (for example assuming boost invariant longitudinal expansion and neglecting transverse expansion) such that we have been able to determine the corrections to the hadron spectra in the final state coming from the hadronization of the wake in the plasma analytically, and without needing to incorporate any additional parameters into our model. By comparing our computations with and without the effects of the backreaction of the medium, we have established that, indeed, these collective effects have important consequences for jet shapes, fragmentation function and missing- $p_{\mathrm{T}}$ observables as discussed in sections 4.3 and section 4.4.

Including the effects of the backreaction of the medium results in enhanced jet shapes at large angles and enhanced fragmentation functions at soft momenta. These effects go in the directions favored by experimental data, but in our treatment they are not as large in magnitude as the data seem to require. It should also be noted that these observables are not dominated by these effects.

The distribution of the missing- $p_{\mathrm{T}}$ observable $\not p_{\mathrm{T}}^{\|}$constructed from dijet events is particularly interesting because at all but the smallest angles it is dominated by effects coming directly from the backreaction of the medium. Even with our simplified approach to the backreaction of the medium and to hadronization, we obtain a rather good qualitative de- 
scription of the shape, $p_{\mathrm{T}^{-}}$-dependence, angular dependence, and $A_{J^{-}}$and $R$-dependence of the $\not p_{\mathrm{T}}^{\|}$distributions, see figure 13 and figure 14. This indicates that our simplified analysis of the backreaction of the medium is on the right track, and confirms the absolute necessity of including this physics if one aims to understand the soft and/or large-angle components of jets as reconstructed in heavy ion collisions.

The main discrepancy that we find between the results of our calculations and the experimentally measured $\not p_{T}^{\|}$distributions is that our calculation does not yield enough semi-hard particles coming from the wake in the plasma in the $2<p_{\mathrm{T}}<4 \mathrm{GeV}$ regime at angles less than $\sim 0.5$ radians from the jet axis. Given that it has momentum conservation built into it, our calculation must overproduce particles in some other regime, and indeed we find that our wake results in somewhat more particles with $p_{\mathrm{T}}<2 \mathrm{GeV}$ than indicated in the data, over an even broader range of angles. Although we cannot be sure of this, we expect that the remaining discrepancies between our results and experimental data on jet shapes and fragmentation functions can also be traced to our calculation not producing enough particles with $2<p_{\mathrm{T}}<4 \mathrm{GeV}$.

We are aware of three possible reasons for the discrepancy that we have found in the semi-hard momentum regime, for momenta well above $T$ but not so high that particles coming from the wake can be neglected. First, we know that our perturbative expression (4.5) for the correction to the spectrum of particles coming from the plasma due to the wake is not valid for $p_{\mathrm{T}}$ well above $T$, and furthermore we know that because it is based on a linear approximation to an exponential the expression (4.5) necessarily underestimates particle production from the wake in this regime. And, even if the modifications of the flow field induced by the wake are small their effect on the spectrum of particles produced at freezeout can be significant on the tail of the thermal distribution. Going beyond our simplified expressions (4.5) and (4.7) in order to improve upon our description of the semihard momentum regime requires taking account of the explicit space-time dependence of the hydrodynamic wake in the plasma [174]. It would be interesting to investigate the effect of the full flow profile as predicted by holographic computations of the hydrodynamic wake $[123,125]$ on the final particle production spectrum to see how this modifies our results in the semi-hard momentum regime.

The second possible reason for the discrepancy that we have found in the semi-hard momentum regime could be that the energy lost by the jet does not fully hydrodynamize. That is, a part of the discrepancy at semi-hard momenta between our results for the $\not p_{\mathrm{T}}^{\|}$ distribution and the experimental results in figure 13 and figure 14 could be a manifestation of non-equilbrium, and non-hydrodynamic, physics in quark-gluon plasma that has been disturbed by the passage of the jet. This possibility can be thought of in two ways that sound different but are operationally equivalent in the present context. One way of describing this possibility is to say that the energy and momentum deposited by the jet in the plasma has not all had time to thermalize as fully as we assume in our simplified analysis. This may be particularly relevant to energy deposited not long before the jet exits the droplet of plasma. Another way of describing the same possibility is to say that our hybrid model neglects medium-induced gluon radiation: in our model, the partons in the jet lose energy to a wake in the plasma and are kicked in transverse momentum but the 
branching structure of the jet shower is not modified in the way that happens in entirely weakly coupled treatments of jet quenching. In a weakly coupled analysis in which energy is lost via medium-induced gluon radiation, a fraction of the energy lost in this way (called $\Delta E_{\text {thermal }}$ in ref. [156]) is carried by radiated gluons that themselves further radiate and cascade down to the medium scale and form a hydrodynamized wake in the plasma [156]. With regards to this component of the energy lost via medium-induced gluon radiation, neglecting the medium-modification of the branching structure of the jet shower as we do in our model makes absolutely no difference: our model includes the loss of energy from the partons in the jet and includes the hydrodynamized wake in the plasma. Where these details will matter is for those radiated gluons that do not thermalize as fully as we assume, as may be particularly relevant to gluons radiated not long before the jet exits the droplet of plasma. The first description uses words that arise in strongly coupled analyses of jet quenching. The second description uses words that arises in weakly coupled analyses. The two are operationally equivalent here.

Another physical process which has not been incorporated into our approach is the effect of imperfect resolution. In our hybrid model as currently implemented, as soon as one parton in the jet shower splits into two, the two daughter partons are treated as separate losers of energy. That is, the medium can resolve the separation of one parton into two as soon as it happens, when the two daughters are still very close to each other. This cannot be correct. In reality, the medium can only resolve two partons as two separate losers of energy after they have separated by some resolution length that can reasonably be expected to be of order the Debye length of the plasma. The main effect that the inclusion of the effect of imperfect resolution would have in our model is that semi-hard partons within jets, in particular within narrow jets, would start losing their energy rapidly on their own later than in our present model, and so would end up less quenched. Hence, our neglect of these effects of imperfect resolution is a third possible reason for the discrepancy that we have found in the semi-hard momentum regime.

It would be very interesting to assess how much of the discrepancy that we find in the semi-hard momentum regime is hydrodynamic (and due to our simplifications), or arises because some of the energy lost by the jet does not fully hydrodynamize, or is related to our neglect of resolution effects. Each explanation is quite interesting in its own right and deserves investigation. We leave this to future work, but note here that this example serves very well to make our point that the most instructive uses of a few-parameter model like the one we have constructed are to discover, and subsequently understand, the ways in which it fails to describe data. This example also serves to highlight the full power of the suite of experimental data on intrajet structure now available, including jet shapes, fragmentation functions, and the missing- $p_{\mathrm{T}}$ observables. We encourage theorists pursuing other approaches to calculate all these observables and compare to data. Consider for example the weakly coupled calculations in which energy is lost to radiated gluons which themselves are quickly degraded down to soft gluons with momenta of order the temperature [150-156], which leads to rapid hydrodynamization of the emitted energy [156]. These approaches yield qualitative agreement with the $\not p_{\mathrm{T}}^{\|}$-distribution that we have shown in figure 12 [152], but fail to reproduce the soft enhancement of the fragmentation function 
unless further soft radiation attributed to decoherence is added [187]. It would be very interesting to see how well they predict the $A_{J}$-dependence and the $R$-dependence of the $\not p_{\mathrm{T}^{-}}^{\|}$ distribution as in figure 13 and figure 14, in particular in the soft and semi-hard momentum regimes. Weakly coupled approaches in which it is assumed that the emitted gluons do not further interact at all are able to reproduce the enhancement of jet shapes at large angles [104]; it would be interesting to analyze the $\not p_{\mathrm{T}}^{\|}$-distributions in these approaches.

Although the physics of the wake in the plasma is of interest in its own right, if in the longer term our goal is to quantify broadening, determine the value of the key medium property $K$, and ultimately to see rare but not-too-rare large angle scattering of partons within jets that may allow us to see the length scale at which strongly coupled quark-gluon plasma emerges from the fluctuations at even smaller length scales that behave as weakly coupled quark and gluon quasiparticles, then we will want to focus on observables sensitive to the angular distribution of $10-20 \mathrm{GeV}$ partons within jets as in figure 7 , where the wake makes no contribution.

\section{A Transverse kicks kinematics}

As a parton propagates though the plasma, it receives a random transverse kick in the local fluid rest frame that we shall denote by $q$. In this appendix, we provide a precise specification of what we mean by this. Denoting the momenta of the parton in the fluid rest frame before and after a transverse kick by $P^{\mu}=E_{F}\left(1, \mathbf{w}_{F}\right)$ and $P^{\prime} \mu=E_{F}\left(1, \mathbf{w}_{F}^{\prime}\right)$, and assuming that the transverse kick serves only to change the direction of $\mathbf{w}_{F}$, meaning $\mathbf{w}_{F}^{\prime 2}=\mathbf{w}_{F}^{2}$, the momentum after the kick is given by

$$
\mathbf{w}_{F}^{\prime}=\sqrt{1-\frac{q^{2}}{E_{F}^{2} \mathbf{w}_{F}^{2}}} \mathbf{w}_{F}+\frac{q}{E_{F}} \mathbf{e}_{\perp},
$$

where $\mathbf{e}_{\perp}$ is a vector perpendicular to the parton velocity in the rest frame of the fluid. Note that the virtuality of the parton has not changed. And, in the fluid rest frame, neither has its energy.

Since our computation is performed in the collision center-of-mass frame, we need to express the acquired momentum in that frame. Let us define the four-vector $W=P / E$, with $E$ the energy of the parton in the collision frame. Denoting the fluid velocity in the collision center-of-mass frame by $u=\gamma_{F}(1, \mathbf{v})$, the energies in the fluid and collision frames are related by $E_{F}=E \gamma_{F}(1-\mathbf{w v})$, with $\mathbf{w}$ the velocity of the parton in the collision frame. We construct a four-vector transverse to the fluid velocity

$$
W_{T}=\frac{1}{W_{F}^{0}}(W-(W \cdot u) u)
$$

which, in the fluid frame, has components $W_{T}=\left(0, \mathbf{w}_{F}\right)$. We use this vector to express the change in four-momentum associated with the kick as

$$
P^{\prime \mu}=P^{\mu}-\beta E_{F} W_{T}^{\mu}+q e_{\perp}^{\mu}, \quad \beta \equiv 1-\sqrt{1-\frac{q^{2}}{E_{F}^{2} \mathbf{w}_{F}^{2}}},
$$


where $E_{F}^{2} \mathbf{w}_{F}^{2}=E_{F}^{2}-E^{2}\left(1-\mathbf{w}^{2}\right)$ and the four-vector $e_{\perp}^{\mu}$ satisfies the conditions

$$
u \cdot e_{\perp}=0, \quad W \cdot e_{\perp}=0, \quad e_{\perp}^{2}=-1 .
$$

It is then possible to show that $e_{\perp}$ can be written as a linear combination of two orthogonal vectors satisfying the conditions

$$
e_{1}^{\mu}=\left(0, \frac{\mathbf{w} \times \mathbf{v}}{|\mathbf{w} \times \mathbf{v}|}\right), \quad e_{2}^{\mu}=\frac{1}{\sqrt{N}}\left(l_{2}^{\mu}+\alpha W_{\perp}^{\mu}\right),
$$

with

$$
\begin{aligned}
l_{2}^{\mu} & =\left(0, \frac{\mathbf{w}}{|\mathbf{w}|} \times \frac{\mathbf{w} \times \mathbf{v}}{|\mathbf{w} \times \mathbf{v}|}\right), & W_{\perp} & =W-\frac{W^{2}}{u \cdot W} u, \\
\alpha & =-\frac{\left(l_{2} \cdot u\right)(u \cdot W)}{(u \cdot W)^{2}-W^{2}}, & N & =\frac{(u \cdot W)^{2}-W^{2}\left(1+\left(l_{2} \cdot u\right)^{2}\right)}{(u \cdot W)^{2}-W^{2}} .
\end{aligned}
$$

These expressions allow us to determine the change in momentum in terms of collision frame quantities alone, once $q$ and the angle in the $\left(e_{1}, e_{2}\right)$ plane have been chosen. After each time interval $d t$ in the collision frame, we select a random value of $q$ according to a Gaussian probability distribution with width $\Delta Q_{\perp}^{2}=\hat{q} d t_{F}$, where the relation between $d t_{F}$ and $d t$ is $d t_{F}=d t \gamma_{F}(1-\mathbf{w v})$. The angle is chosen randomly with a uniform distribution. This procedure is repeated for each parton in the shower as long as it is in a location where the temperature of the expanding cooling hydrodynamic fluid still satisfies $T>T_{c}$.

The kick that the parton receives is transverse only in the local fluid rest frame. In the collision center-of-mass frame, the kick has a transverse component but it also has a longitudinal component which results in energy loss given by

$$
\Delta E=-\beta E \gamma_{F}^{2}\left(\mathbf{v w}-v^{2}\right)-q^{(2)} \frac{|\mathbf{w}||\mathbf{w} \times \mathbf{v}|}{\mathbf{w}^{2}-\mathbf{v w}}
$$

where $q^{(2)}$ is the component of the momentum transfer along the $e_{2}$ direction. (Note that this formula is badly behaved when the velocities of the particle and the fluid are identical, since in that case it is not possible to define the transverse direction in the fluid frame. We have tested that in our simulation this does not occur over the whole propagation of the parton in the plasma.)

\section{B Background subtraction procedure}

As we have described in section 4 , in order to analyze the observable consequences of the wake in the hydrodynamic medium, we need to add a background of particles that reproduces the measured particle yields and spectra so that we can then incorporate the effects of the wake as a perturbation on these spectra. So, our PyтHIA simulations of jet showers, modified as in our hybrid model, are now embedded in a (perturbed) background, meaning that we must run a background subtraction algorithm. We have used an iterative noise/pedestal subtraction procedure similar to the one implemented by the experimental collaborations. Since the algorithms employed by ATLAS and CMS are different, we have 
employed different version of the procedure for the analysis of observables for jets with $p_{T}>100 \mathrm{GeV}$, where we follow CMS [184], and for the observables that cover jets with $p_{T}<100 \mathrm{GeV}$, where we follow ATLAS [48]. See the figures in section 4 for the results we have obtained after following these procedures, procedures which we detail below.

For high $p_{T}$ jet observables, our procedure follows the following steps:

(i) Discretize the $(\eta, \phi)$ space in cells of size $0.091 \times 0.087$. Sum the transverse energy $E_{T}$ of all particles falling into the same cell.

(ii) Compute the average transverse energy and the standard deviation in the transverse energy for all the cells in a strip with a given rapidity, i.e. $\left\langle E_{T}(\eta)\right\rangle$ and $\sigma(\eta) \equiv$ $\sqrt{\left\langle E_{T}^{2}(\eta)\right\rangle-\left\langle E_{T}(\eta)\right\rangle^{2}}$.

(iii) For each cell, subtract the average for the strip in which the cell is found and subtract a contribution proportional to the standard deviation for that strip, with the proportionality constant $\beta$ a parameter that we shall return to below. If the result is negative, set it to zero instead. That is, for the $i$ 'th cell in the strip compute:

$$
\hat{E}_{T}^{i} \equiv \max \left[E_{T}^{i}-\left\langle E_{T}(\eta)\right\rangle-\beta \sigma(\eta), 0\right]
$$

(iv) Run the anti- $k_{t}$ clustering algorithm using all cells whose $\hat{E}_{T}^{i}$ is different from zero. Each such cell is given to the anti- $k_{t}$ algorithm as a null four-vector with transverse momentum $\hat{E}_{T}^{i}$ located at an $\eta$ and $\phi$ corresponding to the geometric center of the cell.

$(v)$ Repeat step (ii) excluding all cells that the anti- $k_{t}$ algorithm has already identified as belonging to a jet with transverse energy above $E_{T}^{\text {cut }}$. Jets with energy above this cut are considered signal jets. We now make a second pass, re-evaluating the background outside these jets.

(vi) Repeat the background subtraction (iii) and the jet finding step $(i v)$ using the values obtained in previous step. The jets found by the anti- $k_{t}$ algorithm on this second pass, which may have transverse energies above or below $E_{T}^{\text {cut }}$, are added to the collection of signal jets for this event.

This procedure involves choosing two parameters, namely $\beta$ and $E_{T}^{\text {cut }}$. The best choice for these parameters will in general depend on the anti- $k_{t}$ reconstruction parameter $R$, the jet $p_{T}$ range under study, and the magnitude of the cell-to-cell fluctuations of the background. The factor $\beta$ controls the effect of background fluctuations. For a homogeneous background with no fluctuations, one should choose $\beta=0$. For a cell-to-cell fluctuating background, one needs to increase $\beta$ accordingly, at the risk of potentially removing some of the signal of interest. The value of $E_{T}^{\text {cut }}$ determines whether a group of cells corresponds to signal and should therefore be excluded from the background estimation. The choice of these two parameters should be guided by the criterion that the $p_{T}$ of a jet that was artificially embedded into the background and then reconstructed upon performing the background subtraction procedure above is, on average, as close as possible to the original $p_{T}$ of the 
artificial jet, within $2 \%$ for jets with $p_{T} \sim 100 \mathrm{GeV}$. This is referred to as ensuring that one has a good jet energy scale (JES). We have done this test using PYTHIA jets. We choose $E_{T}^{\text {cut }}=30 \mathrm{GeV}$ throughout, and have then picked a value of $\beta$ for each centrality and each value of the anti- $k_{t}$ parameter $R$ so as to opimize the JES. For events with $0-10 \%$ centrality, we choose $\beta=0.48,0.72,1.00,1.17$ for $R=0.2,0.3,0.4,0.5$ while for events with $10-30 \%$ centrality the corresponding values that we choose are $\beta=0.42,0.71,0.94,1.13$.

For jets in the lower $p_{T}$ region, with $p_{T}<100 \mathrm{GeV}$, the procedure that we adopt begins with the same discretization of $(\eta, \phi)$ space and then continues as follows:

(i) Reconstruct jets using the anti- $k_{t}$ algorithm for many different values of the reconstruction parameter $R$, in each case using the uncorrected the $E_{T}$ in each cell in $(\eta, \phi)$, introducing null four-vectors with transverse momentum $E_{T}$ with a $\eta$ and $\phi$ corresponding to the geometric center of the cell. Because in this procedure we start by reconstructing jets before subtracting background, after subtracting background we must then also remove combinatorial jets.

(ii) Select a set of seed jets with $R=0.2$ which have at least one constituent cell with $E_{T}>3 \mathrm{GeV}$ and whose cell with the maximum transverse energy $E_{T}^{\max }$ satisfies $E_{T}^{\max }>4\left\langle E_{T}\right\rangle$, where $\left\langle E_{T}\right\rangle$ is the average transverse energy of the cells within the seed jet.

(iii) Compute the average transverse energy of each rapidity strip, i.e. $\left\langle E_{T}(\eta)\right\rangle$, but excluding all those cells that belong to a seed jet.

(iv) Subtract from each cell the average transverse energy for that rapidity strip, $\hat{E}_{T}^{i}=$ $E_{T}^{i}-\left\langle E_{T}(\eta)\right\rangle$. This subtraction is applied to all cells in a strip, including those in a seed jet, meaning that it modifies the $p_{T}$ of the seed jets. This is the first of two subtractions.

(v) The second subtraction, below, will employ a subset of the seed jets with $R=0.2$ from above whose transverse momenta after the first subtraction above satisfy $p_{T}^{\text {jet }}>$ $25 \mathrm{GeV}$. It will also employ a set of seed "track jets" with $p_{T}^{\text {jet }}>10 \mathrm{GeV}$. Track jets are built using only charged tracks with $p_{T}^{\text {track }}>4 \mathrm{GeV}$ and are reconstructed using the anti- $k_{t}$ algorithm with $R=0.4$.

(vi) Recompute the average transverse energy $\left\langle E_{T}^{\prime}(\eta)\right\rangle$, now excluding all those cells that lie within $\Delta r=0.4$ from the axis of any of the seed jets or seed track jets, where $\Delta R$ is the angular distance in $(\eta, \phi)$ space.

(vii) Subtract the new average energy $\left\langle E_{T}^{\prime}(\eta)\right\rangle$ from all cells and update the kinematics of all jets reconstructed with all values of $R$. Keep only those jets with $E_{T}>20 \mathrm{GeV}$.

(viii) In order to suppress the contribution of combinatorial jets, we impose that the reconstructed jets have to lie within $\Delta r=0.2$ of a seed track jet (defined above) with $p_{T}>7 \mathrm{GeV}$, making it probable that the signal jets include one or a few hard particles in them. 
Depending on which observables we want to compute and compare to data, we will need to apply one or more further corrections to the jets in $\mathrm{PbPb}$ events that we have extracted via the background subtraction and jet reconstruction procedures detailed above.

For all observables, we will follow the experimental analyses and apply a JES correction which takes into account the remaining average disagreement between the energy of a proton-proton jet from РүтніA and the energy of that jet after it has been embedded into one of our $\mathrm{PbPb}$ events and reconstructed as above. For the first of the two procedures above, the one that we employ for jets with $p_{T}>100 \mathrm{GeV}$, the JES correction is less than $2 \%$, as we noted above. For the procedure that we employ for jets with $p_{T}<100 \mathrm{GeV}$, the JES correction can be as large as $12 \%$ or $20 \%$ for $R=0.4$ or $R=0.5$ jets with $p_{T}=40 \mathrm{GeV}$.

If we wish to compare to experimental data that has not been unfolded, as we shall do in the case of several high- $p_{\mathrm{T}}$ observables measured by CMS, we must include an additional jet energy resolution (JER) correction. We start by doing a Monte Carlo study in which we insert jets from PYTHIA into an event, fit the distribution of the energy reconstruction efficiency (reconstructed jet energy over generator level jet energy as a function of generator level jet energy) for each jet $p_{T}$ bin with a Gaussian and extract the corresponding standard deviation $\sigma$, which tells us how much the reconstructed jet energy varies from event to event and jet to jet. (This $\sigma$ is unrelated to the $\sigma$ in the background subtraction procedure above; both are conventionally referred to as $\sigma$.) When we do this calculation using the events in our model including its simplified background, we denote what we obtain by $\sigma_{\text {model }}$. CMS has done this analysis on data from heavy ion collisions, obtaining $\sigma_{\mathrm{LHC}}$ which they have tabulated as a function of jet $p_{\mathrm{T}}$ in ref. [46]. We are now ready to correct for the fact that the JER in the real background measured by CMS is different from that in our simplified model background. We do so by smearing the jet energies in our model calculation with a Gaussian whose width corresponds to $\sigma_{\text {extra }} \equiv \sqrt{\sigma_{\text {LHC }}^{2}-\sigma_{\text {model }}^{2}}$.

If we wish to compare to data that has been unfolded, we will perform the simplest version of the so called bin-by-bin unfolding. This affects the jet spectrum measurements. The correction applied consists of multiplying the spectrum of the medium-modified jets that we have reconstructed via one of the two procedures above by the ratio of two other spectra: the jet spectrum obtained directly from PYTHIA divided by the jet spectrum obtained after embedding Pyтнia jets into heavy ion collision events and reconstructing them.

\section{Acknowledgments}

We thank J. Brewer, P. Chesler, Z. Hulcher, P. Jacobs, R. Kunnawalkam Elayavalli, Y.J. Lee, Y. Mehtar-Tani, A. H. Mueller, G. Qin, A. Sadofyev, K. Tywoniuk, W. van der Schee and K. Zapp for helpful discussions. We also thank J. Balewski, M. Goncharov and C. Paus for their assistance with computing. JCS is a Royal Society University Research Fellow. The work of JCS was also supported by a Ramón y Cajal fellowship. The work of JGM was partly supported by Fundação para a Ciência e a Tecnologia (Portugal) under project CERN/FIS-NUC/0049/2015 and contract "Investigador FCT - Development Grant". DP acknowledges the hospitality of the Center for Theoretical Physics, MIT, where part of this work was completed. JCS and DP acknowledge funding from grants FPA2013-46570 and 
MDM-2014-0369 of ICCUB (Unidad de Excelencia 'María de Maeztu') from the Spanish MINECO, from grant 2014-SGR-104 from the Generalitat de Catalunya and from the Consolider CPAN project. KR acknowledges the hospitality of the CERN Theory division during the completion of this work. The work of KR was supported by the U.S. Department of Energy under grant Contract Number DE-SC0011090.

Open Access. This article is distributed under the terms of the Creative Commons Attribution License (CC-BY 4.0), which permits any use, distribution and reproduction in any medium, provided the original author(s) and source are credited.

\section{References}

[1] PHENIX collaboration, K. Adcox et al., Formation of dense partonic matter in relativistic nucleus-nucleus collisions at RHIC: Experimental evaluation by the PHENIX collaboration, Nucl. Phys. A 757 (2005) 184 [nucl-ex/0410003] [INSPIRE].

[2] BRAHMS collaboration, I. Arsene et al., Quark gluon plasma and color glass condensate at RHIC? The Perspective from the BRAHMS experiment, Nucl. Phys. A 757 (2005) 1 [nucl-ex/0410020] [INSPIRE].

[3] B.B. Back et al., The PHOBOS perspective on discoveries at RHIC, Nucl. Phys. A 757 (2005) 28 [nucl-ex/0410022] [INSPIRE].

[4] STAR collaboration, J. Adams et al., Experimental and theoretical challenges in the search for the quark gluon plasma: The STAR Collaboration's critical assessment of the evidence from RHIC collisions, Nucl. Phys. A 757 (2005) 102 [nucl-ex/0501009] [INSPIRE].

[5] ALICE collaboration, Elliptic flow of charged particles in $\mathrm{Pb}-\mathrm{Pb}$ collisions at 2.76 $\mathrm{TeV}$, Phys. Rev. Lett. 105 (2010) 252302 [arXiv:1011.3914] [INSPIRE].

[6] ATLAS collaboration, Measurement of the pseudorapidity and transverse momentum dependence of the elliptic flow of charged particles in lead-lead collisions at $\sqrt{s_{N N}}=2.76$ TeV with the ATLAS detector, Phys. Lett. B 707 (2012) 330 [arXiv:1108.6018] [InSPIRE].

[7] CMS collaboration, Measurement of the elliptic anisotropy of charged particles produced in $\mathrm{PbPb}$ collisions at $\sqrt{s}_{N N}=2.76 \mathrm{TeV}$, Phys. Rev. C 87 (2013) 014902 [arXiv:1204.1409] [INSPIRE].

[8] P. Huovinen, P.F. Kolb, U.W. Heinz, P.V. Ruuskanen and S.A. Voloshin, Radial and elliptic flow at RHIC: Further predictions, Phys. Lett. B 503 (2001) 58 [hep-ph/0101136] [INSPIRE].

[9] D. Teaney, J. Lauret and E.V. Shuryak, A Hydrodynamic description of heavy ion collisions at the SPS and RHIC, nucl-th/0110037 [INSPIRE].

[10] T. Hirano, U.W. Heinz, D. Kharzeev, R. Lacey and Y. Nara, Hadronic dissipative effects on elliptic flow in ultrarelativistic heavy-ion collisions, Phys. Lett. B 636 (2006) 299 [nucl-th/0511046] [INSPIRE].

[11] P. Romatschke and U. Romatschke, Viscosity Information from Relativistic Nuclear Collisions: How Perfect is the Fluid Observed at RHIC?, Phys. Rev. Lett. 99 (2007) 172301 [arXiv:0706.1522] [INSPIRE]. 
[12] M. Luzum and P. Romatschke, Conformal Relativistic Viscous Hydrodynamics: Applications to RHIC results at $\sqrt{s_{N N}}=200 \mathrm{GeV}$, Phys. Rev. C 78 (2008) 034915 [Erratum ibid. C 79 (2009) 039903] [arXiv:0804.4015] [INSPIRE].

[13] B. Schenke, S. Jeon and C. Gale, Elliptic and triangular flow in event-by-event $(3+1) D$ viscous hydrodynamics, Phys. Rev. Lett. 106 (2011) 042301 [arXiv: 1009.3244] [INSPIRE].

[14] T. Hirano, P. Huovinen and Y. Nara, Elliptic flow in $P b+P b$ collisions at $\sqrt{s_{N N}}=2.76$ TeV: hybrid model assessment of the first data, Phys. Rev. C 84 (2011) 011901 [arXiv: 1012.3955] [INSPIRE].

[15] C. Gale, S. Jeon, B. Schenke, P. Tribedy and R. Venugopalan, Event-by-event anisotropic flow in heavy-ion collisions from combined Yang-Mills and viscous fluid dynamics, Phys. Rev. Lett. 110 (2013) 012302 [arXiv: 1209.6330] [INSPIRE].

[16] C. Shen, Z. Qiu, H. Song, J. Bernhard, S. Bass and U. Heinz, The iEBE-VISHNU code package for relativistic heavy-ion collisions, Comput. Phys. Commun. 199 (2016) 61 [arXiv: 1409.8164] [INSPIRE].

[17] C. Shen, J.-F. Paquet, U. Heinz and C. Gale, Photon Emission from a Momentum Anisotropic quark-gluon Plasma, Phys. Rev. C 91 (2015) 014908 [arXiv:1410.3404] [INSPIRE].

[18] J.E. Bernhard, J.S. Moreland, S.A. Bass, J. Liu and U. Heinz, Applying Bayesian parameter estimation to relativistic heavy-ion collisions: simultaneous characterization of the initial state and quark-gluon plasma medium, Phys. Rev. C 94 (2016) 024907 [arXiv: 1605. 03954] [INSPIRE].

[19] CMS collaboration, Observation of Long-Range Near-Side Angular Correlations in Proton-Proton Collisions at the LHC, JHEP 09 (2010) 091 [arXiv: 1009.4122] [INSPIRE].

[20] CMS collaboration, Observation of long-range near-side angular correlations in proton-lead collisions at the LHC, Phys. Lett. B 718 (2013) 795 [arXiv:1210.5482] [INSPIRE].

[21] ALICE collaboration, Long-range angular correlations on the near and away side in $\mathrm{p}-\mathrm{Pb}$ collisions at $\sqrt{s_{N N}}=5.02 \mathrm{TeV}$, Phys. Lett. B 719 (2013) 29 [arXiv:1212.2001] [InSPIRE].

[22] ATLAS collaboration, Observation of Associated Near-Side and Away-Side Long-Range Correlations in $\sqrt{s_{N N}}=5.02$ TeV Proton-Lead Collisions with the ATLAS Detector, Phys. Rev. Lett. 110 (2013) 182302 [arXiv:1212.5198] [INSPIRE].

[23] PHENIX collaboration, A. Adare et al., Quadrupole Anisotropy in Dihadron Azimuthal Correlations in Central $d+A u$ Collisions at $\sqrt{s_{N N}}=200$ GeV, Phys. Rev. Lett. 111 (2013) 212301 [arXiv: 1303.1794] [INSPIRE].

[24] CMS collaboration, Multiplicity and transverse momentum dependence of two- and four-particle correlations in pPb and PbPb collisions, Phys. Lett. B 724 (2013) 213 [arXiv: 1305.0609] [INSPIRE].

[25] CMS collaboration, Evidence for Collective Multiparticle Correlations in p-Pb Collisions, Phys. Rev. Lett. 115 (2015) 012301 [arXiv:1502.05382] [INSPIRE].

[26] PHENIX collaboration, A. Adare et al., Measurements of elliptic and triangular flow in high-multiplicity ${ }^{3} \mathrm{He}+$ Au collisions at $\sqrt{s_{N N}}=200 \mathrm{GeV}$, Phys. Rev. Lett. 115 (2015) 142301 [arXiv: 1507.06273] [INSPIRE]. 
[27] ATLAS collaboration, Observation of Long-Range Elliptic Azimuthal Anisotropies in $\sqrt{s}=13$ and 2.76 TeV pp Collisions with the ATLAS Detector, Phys. Rev. Lett. 116 (2016) 172301 [arXiv: 1509.04776] [INSPIRE].

[28] CMS collaboration, Pseudorapidity dependence of long-range two-particle correlations in $p P b$ collisions at $\sqrt{s_{N N}}=5.02 \mathrm{TeV}$, Submitted to: Phys. Rev. C (2016)

[arXiv: 1604.05347] [INSPIRE].

[29] CMS collaboration, Evidence for collectivity in pp collisions at the LHC, Phys. Lett. B 765 (2017) 193 [arXiv: 1606. 06198] [inSPIRE].

[30] P. Bozek, Collective flow in $p$-Pb and d-Pd collisions at TeV energies, Phys. Rev. C 85 (2012) 014911 [arXiv:1112.0915] [INSPIRE].

[31] J.L. Nagle et al., Exploiting Intrinsic Triangular Geometry in Relativistic He3+Au Collisions to Disentangle Medium Properties, Phys. Rev. Lett. 113 (2014) 112301 [arXiv:1312.4565] [INSPIRE].

[32] B. Schenke and R. Venugopalan, Eccentric protons? Sensitivity of flow to system size and shape in $p+p, p+P b$ and $P b+P b$ collisions, Phys. Rev. Lett. 113 (2014) 102301 [arXiv: 1405.3605] [INSPIRE].

[33] I. Kozlov, M. Luzum, G. Denicol, S. Jeon and C. Gale, Transverse momentum structure of pair correlations as a signature of collective behavior in small collision systems, arXiv:1405.3976 [INSPIRE].

[34] P. Romatschke, Light-Heavy Ion Collisions: A window into pre-equilibrium QCD dynamics?, Eur. Phys. J. C 75 (2015) 305 [arXiv:1502.04745] [INSPIRE].

[35] P. Bozek and W. Broniowski, Hydrodynamic modeling of ${ }^{3}$ He-Au collisions at $\sqrt{s N N}=200$ GeV, Phys. Lett. B 747 (2015) 135 [arXiv:1503.00468] [inSPIRE].

[36] M. Habich, G.A. Miller, P. Romatschke and W. Xiang, Testing hydrodynamic descriptions of $p+p$ collisions at $\sqrt{s}=7 \mathrm{TeV}$, Eur. Phys. J. C 76 (2016) 408 [arXiv:1512.05354] [INSPIRE].

[37] J.D. Orjuela Koop, R. Belmont, P. Yin and J.L. Nagle, Exploring the Beam Energy Dependence of Flow-Like Signatures in Small System d+Au Collisions, Phys. Rev. C 93 (2016) 044910 [arXiv: 1512.06949] [INSPIRE].

[38] P.M. Chesler, Colliding shock waves and hydrodynamics in small systems, Phys. Rev. Lett. 115 (2015) 241602 [arXiv: 1506. 02209] [INSPIRE].

[39] P.M. Chesler, How big are the smallest drops of quark-gluon plasma?, JHEP 03 (2016) 146 [arXiv: 1601.01583] [INSPIRE].

[40] P.M. Chesler and L.G. Yaffe, Holography and colliding gravitational shock waves in asymptotically AdS $S_{5}$ spacetime, Phys. Rev. Lett. 106 (2011) 021601 [arXiv:1011.3562] [INSPIRE].

[41] J. Casalderrey-Solana, M.P. Heller, D. Mateos and W. van der Schee, From full stopping to transparency in a holographic model of heavy ion collisions, Phys. Rev. Lett. 111 (2013) 181601 [arXiv: 1305.4919] [INSPIRE].

[42] P.M. Chesler and L.G. Yaffe, Numerical solution of gravitational dynamics in asymptotically anti-de Sitter spacetimes, JHEP 07 (2014) 086 [arXiv:1309.1439] [INSPIRE]. 
[43] ATLAS collaboration, Observation of a Centrality-Dependent Dijet Asymmetry in Lead-Lead Collisions at $\sqrt{s_{N N}}=2.77 \mathrm{TeV}$ with the ATLAS Detector at the LHC, Phys. Rev. Lett. 105 (2010) 252303 [arXiv: 1011.6182] [INSPIRE].

[44] CMS collaboration, Observation and studies of jet quenching in $\mathrm{PbPb}$ collisions at nucleon-nucleon center-of-mass energy $=2.76$ TeV, Phys. Rev. C 84 (2011) 024906 [arXiv:1102.1957] [INSPIRE].

[45] CMS collaboration, Jet momentum dependence of jet quenching in $\mathrm{PbPb}$ collisions at $\sqrt{s_{N N}}=2.76 \mathrm{TeV}$, Phys. Lett. B 712 (2012) 176 [arXiv:1202.5022] [INSPIRE].

[46] CMS collaboration, Studies of jet quenching using isolated-photon+jet correlations in $\mathrm{PbPb}$ and pp collisions at $\sqrt{s_{N N}}=2.76$ TeV, Phys. Lett. B 718 (2013) 773 [arXiv:1205.0206] [INSPIRE].

[47] CMS collaboration, Measurement of jet fragmentation into charged particles in pp and $\mathrm{PbPb}$ collisions at $\sqrt{s_{N N}}=2.76 \mathrm{TeV}$, JHEP 10 (2012) 087 [arXiv:1205.5872] [INSPIRE].

[48] ATLAS collaboration, Measurement of the jet radius and transverse momentum dependence of inclusive jet suppression in lead-lead collisions at $\sqrt{s_{N N}}=2.76 \mathrm{TeV}$ with the ATLAS detector, Phys. Lett. B 719 (2013) 220 [arXiv:1208.1967] [INSPIRE].

[49] CMS collaboration, Nuclear modification factor of high transverse momentum jets in $\mathrm{PbPb}$ collisions at $\operatorname{sqrt}(\mathrm{sNN})=2.76 \mathrm{TeV}$, CMS-PAS-HIN-12-004.

[50] ATLAS collaboration, Measurement of the Azimuthal Angle Dependence of Inclusive Jet Yields in $\mathrm{Pb}+\mathrm{Pb}$ Collisions at $\sqrt{s_{N N}}=2.76 \mathrm{TeV}$ with the ATLAS detector, Phys. Rev. Lett. 111 (2013) 152301 [arXiv: 1306.6469] [INSPIRE].

[51] CMS collaboration, Modification of jet shapes in PbPb collisions at $\sqrt{s_{N N}}=2.76 \mathrm{TeV}$, Phys. Lett. B 730 (2014) 243 [arXiv:1310.0878] [INSPIRE].

[52] ALICE collaboration, Measurement of charged jet suppression in $\mathrm{Pb}-\mathrm{Pb}$ collisions at $\sqrt{s_{N N}}=2.76 \mathrm{TeV}$, JHEP 03 (2014) 013 [arXiv: 1311.0633] [INSPIRE].

[53] CMS collaboration, Evidence of b-Jet Quenching in PbPb Collisions at $\sqrt{s_{N N}}=2.76 \mathrm{TeV}$, Phys. Rev. Lett. 113 (2014) 132301 [arXiv:1312.4198] [InSPIRE].

[54] CMS collaboration, Measurement of jet fragmentation in $\mathrm{PbPb}$ and $p p$ collisions at $\sqrt{s_{N N}}=2.76 \mathrm{TeV}$, Phys. Rev. C 90 (2014) 024908 [arXiv: 1406.0932] [INSPIRE].

[55] ATLAS collaboration, Measurement of inclusive jet charged-particle fragmentation functions in $\mathrm{Pb}+\mathrm{Pb}$ collisions at $\sqrt{s_{N N}}=2.76 \mathrm{TeV}$ with the ATLAS detector, Phys. Lett. $\mathbf{B}$ 739 (2014) 320 [arXiv:1406.2979] [INSPIRE].

[56] ATLAS collaboration, Measurements of the Nuclear Modification Factor for Jets in $\mathrm{Pb}+\mathrm{Pb}$ Collisions at $\sqrt{s_{\mathrm{NN}}}=2.76 \mathrm{TeV}$ with the ATLAS Detector, Phys. Rev. Lett. 114 (2015) 072302 [arXiv: 1411.2357] [INSPIRE].

[57] ALICE collaboration, Measurement of jet suppression in central $\mathrm{Pb}-\mathrm{Pb}$ collisions at $\sqrt{s_{\mathrm{NN}}}=2.76 \mathrm{TeV}$, Phys. Lett. B 746 (2015) 1 [arXiv: 1502.01689] [INSPIRE].

[58] ALICE collaboration, Measurement of jet quenching with semi-inclusive hadron-jet distributions in central Pb-Pb collisions at $\sqrt{s_{\mathrm{NN}}}=2.76 \mathrm{TeV}$, JHEP 09 (2015) 170 [arXiv: 1506.03984] [INSPIRE]. 
[59] ATLAS collaboration, Measurement of the production of neighbouring jets in lead-lead collisions at $\sqrt{s_{\mathrm{NN}}}=2.76$ TeV with the ATLAS detector, Phys. Lett. B 751 (2015) 376 [arXiv:1506.08656] [INSPIRE].

[60] CMS collaboration, Measurement of transverse momentum relative to dijet systems in $\mathrm{PbPb}$ and pp collisions at $\sqrt{s_{\mathrm{NN}}}=2.76 \mathrm{TeV}$, JHEP 01 (2016) 006 [arXiv:1509.09029] [INSPIRE].

[61] CMS collaboration, Correlations between jets and charged particles in $\mathrm{PbPb}$ and $p p$ collisions at $\sqrt{s_{\mathrm{NN}}}=2.76 \mathrm{TeV}, \mathrm{JHEP} 02$ (2016) 156 [arXiv:1601.00079] [INSPIRE].

[62] PHENIX collaboration, K. Adcox et al., Suppression of hadrons with large transverse momentum in central Au+Au collisions at $\sqrt{s_{N N}}=130-G e V$, Phys. Rev. Lett. 88 (2002) 022301 [nucl-ex/0109003] [INSPIRE].

[63] STAR collaboration, C. Adler et al., Centrality dependence of high $p_{T}$ hadron suppression in $A u+A u$ collisions at $\sqrt{s}_{N N}=130-G e V$, Phys. Rev. Lett. 89 (2002) 202301 [nucl-ex/0206011] [INSPIRE].

[64] STAR collaboration, C. Adler et al., Disappearance of back-to-back high $p_{T}$ hadron correlations in central Au+Au collisions at $\sqrt{s_{N N}}=200 \mathrm{GeV}$, Phys. Rev. Lett. 90 (2003) 082302 [nucl-ex/0210033] [INSPIRE].

[65] STAR collaboration, M. Ploskon, Inclusive cross section and correlations of fully reconstructed jets in $\sqrt{s_{N N}}=200 \mathrm{GeV} A u+A u$ and $p+p$ collisions, Nucl. Phys. A 830 (2009) 255C-258C [arXiv:0908.1799] [INSPIRE].

[66] PHENIX collaboration, D.V. Perepelitsa, Reconstructed Jet Results in $p+p, d+A u$ and $\mathrm{Cu}+\mathrm{Cu}$ collisions at $200 \mathrm{GeV}$ from PHENIX, Nucl. Phys. A 910-911 (2013) 425.

[67] STAR collaboration, L. Adamczyk et al., Jet-Hadron Correlations in $\sqrt{s_{N N}}=200$ GeV $p+p$ and Central Au+Au Collisions, Phys. Rev. Lett. 112 (2014) 122301 [arXiv: 1302.6184] [INSPIRE].

[68] STAR collaboration, P.M. Jacobs and A. Schmah, Measurements of jet quenching with semi-inclusive charged jet distributions in Au+Au collisions at $\sqrt{s_{N N}}=200$ GeV, Nucl. Phys. A 956 (2016) 641 [arXiv:1512.08784] [InSPIRE].

[69] A. Adare et al., An Upgrade Proposal from the PHENIX Collaboration, arXiv:1501.06197 [INSPIRE].

[70] P. Jacobs and X.-N. Wang, Matter in extremis: Ultrarelativistic nuclear collisions at RHIC, Prog. Part. Nucl. Phys. 54 (2005) 443 [hep-ph/0405125] [INSPIRE].

[71] J. Casalderrey-Solana and C.A. Salgado, Introductory lectures on jet quenching in heavy ion collisions, Acta Phys. Polon. B 38 (2007) 3731 [arXiv:0712.3443] [InSPIRE].

[72] A. Majumder and M. Van Leeuwen, The Theory and Phenomenology of Perturbative QCD Based Jet Quenching, Prog. Part. Nucl. Phys. 66 (2011) 41 [arXiv:1002.2206] [InSPIRE].

[73] J. Ghiglieri and D. Teaney, Parton energy loss and momentum broadening at NLO in high temperature QCD plasmas, Int. J. Mod. Phys. E 24 (2015) 1530013 [arXiv:1502.03730] [INSPIRE].

[74] J.-P. Blaizot and Y. Mehtar-Tani, Jet Structure in Heavy Ion Collisions, Int. J. Mod. Phys. E 24 (2015) 1530012 [arXiv:1503.05958] [InSPIRE].

[75] G.-Y. Qin and X.-N. Wang, Jet quenching in high-energy heavy-ion collisions, Int. J. Mod. Phys. E 24 (2015) 1530014 [arXiv:1511.00790] [INSPIRE]. 
[76] K. Zapp, J. Stachel and U.A. Wiedemann, A Local Monte Carlo implementation of the non-abelian Landau-Pomerantschuk-Migdal effect, Phys. Rev. Lett. 103 (2009) 152302 [arXiv:0812.3888] [INSPIRE].

[77] K. Zapp, G. Ingelman, J. Rathsman, J. Stachel and U.A. Wiedemann, A Monte Carlo Model for 'Jet Quenching', Eur. Phys. J. C 60 (2009) 617 [arXiv:0804.3568] [INSPIRE].

[78] N. Armesto, L. Cunqueiro and C.A. Salgado, Q-PYTHIA: A Medium-modified implementation of final state radiation, Eur. Phys. J. C 63 (2009) 679 [arXiv:0907.1014] [INSPIRE].

[79] B. Schenke, C. Gale and S. Jeon, MARTINI: An Event generator for relativistic heavy-ion collisions, Phys. Rev. C 80 (2009) 054913 [arXiv:0909.2037] [INSPIRE].

[80] I.P. Lokhtin, A.V. Belyaev and A.M. Snigirev, Jet quenching pattern at LHC in PYQUEN model, Eur. Phys. J. C 71 (2011) 1650 [arXiv:1103.1853] [INSPIRE].

[81] K.C. Zapp, F. Krauss and U.A. Wiedemann, A perturbative framework for jet quenching, JHEP 03 (2013) 080 [arXiv: 1212.1599] [INSPIRE].

[82] K.C. Zapp, JEWEL 2.0.0: directions for use, Eur. Phys. J. C 74 (2014) 2762 [arXiv:1311.0048] [INSPIRE].

[83] K.C. Zapp, Geometrical aspects of jet quenching in JEWEL, Phys. Lett. B 735 (2014) 157 [arXiv: 1312.5536] [INSPIRE].

[84] I. Vitev and B.-W. Zhang, Jet tomography of high-energy nucleus-nucleus collisions at next-to-leading order, Phys. Rev. Lett. 104 (2010) 132001 [arXiv:0910.1090] [INSPIRE].

[85] J. Casalderrey-Solana, J.G. Milhano and U.A. Wiedemann, Jet Quenching via Jet Collimation, J. Phys. G 38 (2011) 035006 [arXiv:1012.0745] [INSPIRE].

[86] G.-Y. Qin and B. Müller, Explanation of Di-jet asymmetry in Pb+Pb collisions at the Large Hadron Collider, Phys. Rev. Lett. 106 (2011) 162302 [Erratum ibid. 108 (2012) 189904] [arXiv:1012.5280] [INSPIRE].

[87] C. Young, B. Schenke, S. Jeon and C. Gale, Dijet asymmetry at the energies available at the CERN Large Hadron Collider, Phys. Rev. C 84 (2011) 024907 [arXiv:1103.5769] [INSPIRE].

[88] Y. He, I. Vitev and B.-W. Zhang, $\mathcal{O}\left(\alpha_{s}^{3}\right)$ Analysis of Inclusive Jet and di-Jet Production in Heavy Ion Reactions at the Large Hadron Collider, Phys. Lett. B 713 (2012) 224 [arXiv:1105.2566] [INSPIRE].

[89] J. Casalderrey-Solana, J.G. Milhano and U. Wiedemann, Jet quenching via jet collimation, J. Phys. G 38 (2011) 124086 [arXiv:1107.1964] [InSPIRE].

[90] T. Renk, On the sensitivity of the dijet asymmetry to the physics of jet quenching, Phys. Rev. C 85 (2012) 064908 [arXiv:1202.4579] [INSPIRE].

[91] R.B. Neufeld and I. Vitev, The $Z^{0}$-tagged jet event asymmetry in heavy-ion collisions at the CERN Large Hadron Collider, Phys. Rev. Lett. 108 (2012) 242001 [arXiv:1202.5556] [INSPIRE].

[92] T. Renk, Energy dependence of the dijet imbalance in Pb-Pb collisions at 2.76 ATeV, Phys. Rev. C 86 (2012) 061901 [arXiv: 1204.5572] [INSPIRE]. 
[93] W. Dai, I. Vitev and B.-W. Zhang, Momentum imbalance of isolated photon-tagged jet production at RHIC and LHC, Phys. Rev. Lett. 110 (2013) 142001 [arXiv:1207.5177] [INSPIRE].

[94] L. Apolinario, N. Armesto and L. Cunqueiro, An analysis of the influence of background subtraction and quenching on jet observables in heavy-ion collisions, JHEP 02 (2013) 022 [arXiv:1211.1161] [INSPIRE].

[95] X.-N. Wang and Y. Zhu, Medium Modification of $\gamma$-jets in High-energy Heavy-ion Collisions, Phys. Rev. Lett. 111 (2013) 062301 [arXiv:1302.5874] [INSPIRE].

[96] G.-L. Ma, Dijet asymmetry in $P b+P b$ collisions at $\sqrt{s_{N N}}=2.76$ TeV within a multiphase transport model, Phys. Rev. C 87 (2013) 064901 [arXiv:1304.2841] [INSPIRE].

[97] J. Huang, Z.-B. Kang and I. Vitev, Inclusive b-jet production in heavy ion collisions at the LHC, Phys. Lett. B 726 (2013) 251 [arXiv:1306.0909] [INSPIRE].

[98] F. Senzel, O. Fochler, J. Uphoff, Z. Xu and C. Greiner, Influence of multiple in-medium scattering processes on the momentum imbalance of reconstructed di-jets, J. Phys. $\mathbf{G} 42$ (2015) 115104 [arXiv:1309.1657] [INSPIRE].

[99] R. Perez-Ramos and T. Renk, In-medium jet shape from energy collimation in parton showers: Comparison with CMS PbPb data at 2.76 TeV, Phys. Rev. D 90 (2014) 014018 [arXiv: 1401.5283] [INSPIRE].

[100] T. Renk, A study of the constraining power of high $p_{T}$ observables in heavy-ion collisions, arXiv: 1408.6684 [INSPIRE].

[101] R. Pérez-Ramos and T. Renk, A Monte Carlo study of jet fragmentation functions in $\mathrm{PbPb}$ and pp collisions at $\sqrt{s}=2.76 \mathrm{TeV}$, arXiv:1411.1983 [INSPIRE].

[102] Y.-T. Chien, A. Emerman, Z.-B. Kang, G. Ovanesyan and I. Vitev, Jet Quenching from QCD Evolution, Phys. Rev. D 93 (2016) 074030 [arXiv:1509.02936] [INSPIRE].

[103] J. Huang, Z.-B. Kang, I. Vitev and H. Xing, Photon-tagged and B-meson-tagged b-jet production at the LHC, Phys. Lett. B 750 (2015) 287 [arXiv: 1505.03517] [INSPIRE].

[104] Y.-T. Chien and I. Vitev, Towards the understanding of jet shapes and cross sections in heavy ion collisions using soft-collinear effective theory, JHEP 05 (2016) 023 [arXiv: 1509.07257] [INSPIRE].

[105] J.G. Milhano and K.C. Zapp, Origins of the di-jet asymmetry in heavy ion collisions, Eur. Phys. J. C 76 (2016) 288 [arXiv:1512.08107] [INSPIRE].

[106] X. Zhang, L. Apolinário, J.G. Milhano and M. Płoskoń, Sub-jet structure as a discriminating quenching probe, Nucl. Phys. A 956 (2016) 597 [arXiv:1512.09255] [INSPIRE].

[107] N.-B. Chang and G.-Y. Qin, Full jet evolution in quark-gluon plasma and nuclear modification of jet production and jet shape in $\mathrm{Pb}+\mathrm{Pb}$ collisions at 2.76ATeV at the CERN Large Hadron Collider, Phys. Rev. C 94 (2016) 024902 [arXiv:1603.01920] [INSPIRE].

[108] A.H. Mueller, B. Wu, B.-W. Xiao and F. Yuan, Probing Transverse Momentum Broadening in Heavy Ion Collisions, Phys. Lett. B 763 (2016) 208 [arXiv: 1604.04250] [inSPIRE].

[109] L. Chen, G.-Y. Qin, S.-Y. Wei, B.-W. Xiao and H.-Z. Zhang, Probing Transverse Momentum Broadening via Dihadron and Hadron-jet Angular Correlations in Relativistic Heavy-ion Collisions, arXiv:1607.01932 [INSPIRE]. 
[110] J.M. Maldacena, The Large- $N$ limit of superconformal field theories and supergravity, Int. J. Theor. Phys. 38 (1999) 1113 [hep-th/9711200] [INSPIRE].

[111] J. Casalderrey-Solana, H. Liu, D. Mateos, K. Rajagopal and U.A. Wiedemann, Gauge/String Duality, Hot QCD and Heavy Ion Collisions, arXiv:1101.0618 [INSPIRE].

[112] O. DeWolfe, S.S. Gubser, C. Rosen and D. Teaney, Heavy ions and string theory, Prog. Part. Nucl. Phys. 75 (2014) 86 [arXiv:1304.7794] [InSPIRE].

[113] P.M. Chesler and W. van der Schee, Early thermalization, hydrodynamics and energy loss in AdS/CFT, Int. J. Mod. Phys. E 24 (2015) 1530011 [arXiv:1501.04952] [InSPIRE].

[114] C.P. Herzog, A. Karch, P. Kovtun, C. Kozcaz and L.G. Yaffe, Energy loss of a heavy quark moving through $\mathcal{N}=4$ supersymmetric Yang-Mills plasma, JHEP 07 (2006) 013 [hep-th/0605158] [INSPIRE].

[115] H. Liu, K. Rajagopal and U.A. Wiedemann, Calculating the jet quenching parameter from AdS/CFT, Phys. Rev. Lett. 97 (2006) 182301 [hep-ph/0605178] [INSPIRE].

[116] J. Casalderrey-Solana and D. Teaney, Heavy quark diffusion in strongly coupled $\mathcal{N}=4$ Yang-Mills, Phys. Rev. D 74 (2006) 085012 [hep-ph/0605199] [InSPIRE].

[117] S.S. Gubser, Drag force in AdS/CFT, Phys. Rev. D 74 (2006) 126005 [hep-th/0605182] [INSPIRE].

[118] H. Liu, K. Rajagopal and U.A. Wiedemann, An AdS/CFT Calculation of Screening in a Hot Wind, Phys. Rev. Lett. 98 (2007) 182301 [hep-ph/0607062] [INSPIRE].

[119] M. Chernicoff, J.A. Garcia and A. Guijosa, The Energy of a Moving Quark-Antiquark Pair in an $\mathcal{N}=4$ SYM Plasma, JHEP 09 (2006) 068 [hep-th/0607089] [INSPIRE].

[120] H. Liu, K. Rajagopal and U.A. Wiedemann, Wilson loops in heavy ion collisions and their calculation in AdS/CFT, JHEP 03 (2007) 066 [hep-ph/0612168] [INSPIRE].

[121] S.S. Gubser, Momentum fluctuations of heavy quarks in the gauge-string duality, Nucl. Phys. B 790 (2008) 175 [hep-th/0612143] [InSPIRE].

[122] J. Casalderrey-Solana and D. Teaney, Transverse Momentum Broadening of a Fast Quark in a $\mathcal{N}=4$ Yang-Mills Plasma, JHEP 04 (2007) 039 [hep-th/0701123] [INSPIRE].

[123] P.M. Chesler and L.G. Yaffe, The Wake of a quark moving through a strongly-coupled plasma, Phys. Rev. Lett. 99 (2007) 152001 [arXiv:0706.0368] [INSPIRE].

[124] S.S. Gubser, S.S. Pufu and A. Yarom, Sonic booms and diffusion wakes generated by a heavy quark in thermal AdS/CFT, Phys. Rev. Lett. 100 (2008) 012301 [arXiv:0706.4307] [INSPIRE].

[125] P.M. Chesler and L.G. Yaffe, The Stress-energy tensor of a quark moving through a strongly-coupled $N=4$ supersymmetric Yang-Mills plasma: Comparing hydrodynamics and AdS/CFT, Phys. Rev. D 78 (2008) 045013 [arXiv:0712.0050] [INSPIRE].

[126] D.M. Hofman and J. Maldacena, Conformal collider physics: Energy and charge correlations, JHEP 05 (2008) 012 [arXiv: 0803.1467] [INSPIRE].

[127] S.S. Gubser, D.R. Gulotta, S.S. Pufu and F.D. Rocha, Gluon energy loss in the gauge-string duality, JHEP 10 (2008) 052 [arXiv: 0803.1470] [INSPIRE].

[128] Y. Hatta, E. Iancu and A.H. Mueller, Jet evolution in the $N=4$ SYM plasma at strong coupling, JHEP 05 (2008) 037 [arXiv:0803.2481] [INSPIRE]. 
[129] F. Dominguez, C. Marquet, A.H. Mueller, B. Wu and B.-W. Xiao, Comparing energy loss and p-perpendicular - broadening in perturbative $Q C D$ with strong coupling $N=4 S Y M$ theory, Nucl. Phys. A 811 (2008) 197 [arXiv:0803.3234] [INSPIRE].

[130] P.M. Chesler, K. Jensen and A. Karch, Jets in strongly-coupled $\mathcal{N}=4$ super Yang-Mills theory, Phys. Rev. D 79 (2009) 025021 [arXiv: 0804.3110] [INSPIRE].

[131] P.M. Chesler, K. Jensen, A. Karch and L.G. Yaffe, Light quark energy loss in strongly-coupled $\mathcal{N}=4$ supersymmetric Yang-Mills plasma, Phys. Rev. D 79 (2009) 125015 [arXiv:0810.1985] [INSPIRE].

[132] F. D'Eramo, H. Liu and K. Rajagopal, Transverse Momentum Broadening and the Jet Quenching Parameter, Redux, Phys. Rev. D 84 (2011) 065015 [arXiv:1006.1367] [INSPIRE].

[133] P. Arnold and D. Vaman, Jet quenching in hot strongly coupled gauge theories revisited: 3-point correlators with gauge-gravity duality, JHEP 10 (2010) 099 [arXiv:1008.4023] [INSPIRE].

[134] P. Arnold and D. Vaman, Jet quenching in hot strongly coupled gauge theories simplified, JHEP 04 (2011) 027 [arXiv:1101.2689] [INSPIRE].

[135] P. Arnold and D. Vaman, Some new results for 'jet' stopping in AdS/CFT: long version, J. Phys. G 38 (2011) 124175 [arXiv:1106.1680] [INSPIRE].

[136] M. Chernicoff, J.A. Garcia, A. Guijosa and J.F. Pedraza, Holographic Lessons for Quark Dynamics, J. Phys. G 39 (2012) 054002 [arXiv:1111.0872] [InSPIRE].

[137] P.M. Chesler, Y.-Y. Ho and K. Rajagopal, Shining a Gluon Beam Through quark-gluon Plasma, Phys. Rev. D 85 (2012) 126006 [arXiv:1111.1691] [INSPIRE].

[138] P. Arnold, P. Szepietowski and D. Vaman, Coupling dependence of jet quenching in hot strongly-coupled gauge theories, JHEP 07 (2012) 024 [arXiv:1203.6658] [INSPIRE].

[139] P. Arnold, P. Szepietowski, D. Vaman and G. Wong, Tidal stretching of gravitons into classical strings: application to jet quenching with AdS/CFT, JHEP 02 (2013) 130 [arXiv:1212.3321] [INSPIRE].

[140] P.M. Chesler, M. Lekaveckas and K. Rajagopal, Heavy quark energy loss far from equilibrium in a strongly coupled collision, JHEP 10 (2013) 013 [arXiv:1306. 0564] [INSPIRE].

[141] A. Ficnar and S.S. Gubser, Finite momentum at string endpoints, Phys. Rev. D 89 (2014) 026002 [arXiv: 1306.6648] [INSPIRE].

[142] A. Ficnar, S.S. Gubser and M. Gyulassy, Shooting String Holography of Jet Quenching at RHIC and LHC, Phys. Lett. B 738 (2014) 464 [arXiv:1311.6160] [INSPIRE].

[143] P.M. Chesler and K. Rajagopal, Jet quenching in strongly coupled plasma, Phys. Rev. D 90 (2014) 025033 [arXiv: 1402.6756] [INSPIRE].

[144] R. Rougemont, A. Ficnar, S. Finazzo and J. Noronha, Energy loss, equilibration and thermodynamics of a baryon rich strongly coupled quark-gluon plasma, JHEP 04 (2016) 102 [arXiv: 1507.06556] [INSPIRE].

[145] P.M. Chesler and K. Rajagopal, On the Evolution of Jet Energy and Opening Angle in Strongly Coupled Plasma, JHEP 05 (2016) 098 [arXiv:1511.07567] [INSPIRE]. 
[146] J. Casalderrey-Solana and A. Ficnar, Holographic Three-Jet Events in Strongly Coupled $N=4$ Yang-Mills Plasma, arXiv:1512.00371 [INSPIRE].

[147] K. Rajagopal, A.V. Sadofyev and W. van der Schee, Evolution of the jet opening angle distribution in holographic plasma, Phys. Rev. Lett. 116 (2016) 211603 [arXiv:1602.04187] [INSPIRE].

[148] J. Casalderrey-Solana, D.C. Gulhan, J.G. Milhano, D. Pablos and K. Rajagopal, A Hybrid Strong/Weak Coupling Approach to Jet Quenching, JHEP 10 (2014) 019 [Erratum ibid. 1509 (2015) 175] [arXiv:1405.3864] [INSPIRE].

[149] J. Casalderrey-Solana, D.C. Gulhan, J.G. Milhano, D. Pablos and K. Rajagopal, Predictions for Boson-Jet Observables and Fragmentation Function Ratios from a Hybrid Strong/Weak Coupling Model for Jet Quenching, JHEP 03 (2016) 053 [arXiv:1508.00815] [InSPIRE].

[150] J.-P. Blaizot, E. Iancu and Y. Mehtar-Tani, Medium-induced QCD cascade: democratic branching and wave turbulence, Phys. Rev. Lett. 111 (2013) 052001 [arXiv:1301.6102] [INSPIRE].

[151] A. Kurkela and U.A. Wiedemann, Picturing perturbative parton cascades in QCD matter, Phys. Lett. B 740 (2015) 172 [arXiv:1407.0293] [InSPIRE].

[152] J.-P. Blaizot, Y. Mehtar-Tani and M.A.C. Torres, Angular structure of the in-medium QCD cascade, Phys. Rev. Lett. 114 (2015) 222002 [arXiv:1407.0326] [INSPIRE].

[153] L. Fister and E. Iancu, Medium-induced jet evolution: wave turbulence and energy loss, JHEP 03 (2015) 082 [arXiv:1409.2010] [INSPIRE].

[154] J.-P. Blaizot, L. Fister and Y. Mehtar-Tani, Angular distribution of medium-induced QCD cascades, Nucl. Phys. A 940 (2015) 67 [arXiv:1409.6202] [InSPIRE].

[155] J.-P. Blaizot and Y. Mehtar-Tani, Energy flow along the medium-induced parton cascade, Annals Phys. 368 (2016) 148 [arXiv:1501.03443] [InSPIRE].

[156] E. Iancu and B. Wu, Thermalization of mini-jets in a quark-gluon plasma, JHEP 10 (2015) 155 [arXiv:1506.07871] [INSPIRE].

[157] R.B. Neufeld and I. Vitev, Parton showers as sources of energy-momentum deposition in the QGP and their implication for shockwave formation at RHIC and at the LHC, Phys. Rev. C 86 (2012) 024905 [arXiv:1105.2067] [INSPIRE].

[158] Y. Tachibana and T. Hirano, Momentum transport away from a jet in an expanding nuclear medium, Phys. Rev. C 90 (2014) 021902 [arXiv:1402.6469] [InSPIRE].

[159] S. Floerchinger and K.C. Zapp, Hydrodynamics and Jets in Dialogue, Eur. Phys. J. C 74 (2014) 3189 [arXiv:1407.1782] [INSPIRE].

[160] Y. He, T. Luo, X.-N. Wang and Y. Zhu, Linear Boltzmann Transport for Jet Propagation in the quark-gluon Plasma: Elastic Processes and Medium Recoil, Phys. Rev. C 91 (2015) 054908 [arXiv: 1503.03313] [INSPIRE].

[161] Y. Tachibana and T. Hirano, Interplay between Mach cone and radial expansion and its signal in $\gamma$-jet events, Phys. Rev. C 93 (2016) 054907 [arXiv:1510.06966] [INSPIRE].

[162] S. Cao, T. Luo, G.-Y. Qin and X.-N. Wang, Linearized Boltzmann transport model for jet propagation in the quark-gluon plasma: Heavy quark evolution, Phys. Rev. C 94 (2016) 014909 [arXiv: 1605.06447] [INSPIRE]. 
[163] J. Casalderrey-Solana, J.G. Milhano and P. Quiroga-Arias, Out of Medium Fragmentation from Long-Lived Jet Showers, Phys. Lett. B 710 (2012) 175 [arXiv:1111.0310] [InSPIRE].

[164] T. Sjöstrand, S. Mrenna and P.Z. Skands, A Brief Introduction to PYTHIA 8.1, Comput. Phys. Commun. 178 (2008) 852 [arXiv:0710.3820] [INSPIRE].

[165] JET collaboration, K.M. Burke et al., Extracting the jet transport coefficient from jet quenching in high-energy heavy-ion collisions, Phys. Rev. C 90 (2014) 014909 [arXiv: 1312.5003] [INSPIRE].

[166] J. Casalderrey-Solana, Y. Mehtar-Tani, C.A. Salgado and K. Tywoniuk, New picture of jet quenching dictated by color coherence, Phys. Lett. B 725 (2013) 357 [arXiv:1210.7765] [INSPIRE].

[167] M. Cacciari, G.P. Salam and G. Soyez, The Anti- $k_{t}$ jet clustering algorithm, JHEP 04 (2008) 063 [arXiv: 0802.1189] [INSPIRE].

[168] M. Cacciari, G.P. Salam and G. Soyez, FastJet User Manual, Eur. Phys. J. C 72 (2012) 1896 [arXiv:1111.6097] [INSPIRE].

[169] CMS collaboration, Decomposing energy balance contributions for quenched jets in $\mathrm{PbPb}$ versus pp collisions at $\sqrt{s_{N N}}=2.76 \mathrm{TeV}$, CMS-PAS-HIN-15-011.

[170] F. D'Eramo, M. Lekaveckas, H. Liu and K. Rajagopal, Momentum Broadening in Weakly Coupled quark-gluon Plasma (with a view to finding the quasiparticles within liquid quark-gluon plasma), JHEP 05 (2013) 031 [arXiv:1211.1922] [INSPIRE].

[171] H. Stoecker, Collective flow signals the quark gluon plasma, Nucl. Phys. A 750 (2005) 121 [nucl-th/0406018] [INSPIRE].

[172] J. Casalderrey-Solana, E.V. Shuryak and D. Teaney, Conical flow induced by quenched QCD jets, J. Phys. Conf. Ser. 27 (2005) 22 [hep-ph/0411315] [InSPIRE].

[173] L.M. Satarov, H. Stoecker and I.N. Mishustin, Mach shocks induced by partonic jets in expanding quark-gluon plasma, Phys. Lett. B 627 (2005) 64 [hep-ph/0505245] [INSPIRE].

[174] J. Casalderrey-Solana, E.V. Shuryak and D. Teaney, Hydrodynamic flow from fast particles, hep-ph/0602183 [INSPIRE].

[175] B. Betz, M. Gyulassy, D.H. Rischke, H. Stocker and G. Torrieri, Jet Propagation and Mach Cones in (3+1)d Ideal Hydrodynamics, J. Phys. G 35 (2008) 104106 [arXiv:0804.4408] [INSPIRE].

[176] B. Betz, J. Noronha, G. Torrieri, M. Gyulassy, I. Mishustin and D.H. Rischke, Universality of the Diffusion Wake from Stopped and Punch-Through Jets in Heavy-Ion Collisions, Phys. Rev. C 79 (2009) 034902 [arXiv: 0812.4401] [INSPIRE].

[177] F. Cooper and G. Frye, Comment on the Single Particle Distribution in the Hydrodynamic and Statistical Thermodynamic Models of Multiparticle Production, Phys. Rev. D 10 (1974) 186 [INSPIRE].

[178] ALICE collaboration, Centrality dependence of $\pi, K, p$ production in $\mathrm{Pb}$ - $\mathrm{Pb}$ collisions at $\sqrt{s_{N N}}=2.76 \mathrm{TeV}$, Phys. Rev. C 88 (2013) 044910 [arXiv:1303.0737] [INSPIRE].

[179] A. Beraudo, J.G. Milhano and U.A. Wiedemann, Medium-induced color flow softens hadronization, Phys. Rev. C 85 (2012) 031901 [arXiv:1109.5025] [INSPIRE].

[180] A. Beraudo, J.G. Milhano and U.A. Wiedemann, The Contribution of Medium-Modified Color Flow to Jet Quenching, JHEP 07 (2012) 144 [arXiv:1204.4342] [INSPIRE]. 
[181] M. Cacciari and G.P. Salam, Pileup subtraction using jet areas, Phys. Lett. B 659 (2008) 119 [arXiv: 0707.1378] [InSPIRE].

[182] M. Cacciari, G.P. Salam and G. Soyez, The Catchment Area of Jets, JHEP 04 (2008) 005 [arXiv:0802.1188] [INSPIRE].

[183] M. Cacciari, J. Rojo, G.P. Salam and G. Soyez, Jet Reconstruction in Heavy Ion Collisions, Eur. Phys. J. C 71 (2011) 1539 [arXiv:1010.1759] [INSPIRE].

[184] O. Kodolova, I. Vardanian, A. Nikitenko and A. Oulianov, The performance of the jet identification and reconstruction in heavy ions collisions with CMS detector, Eur. Phys. J. C 50 (2007) 117 [INSPIRE].

[185] S.D. Ellis, J. Huston, K. Hatakeyama, P. Loch and M. Tonnesmann, Jets in hadron-hadron collisions, Prog. Part. Nucl. Phys. 60 (2008) 484 [arXiv:0712.2447] [InSPIRE].

[186] D. d'Enterria, K.J. Eskola, I. Helenius and H. Paukkunen, Confronting current NLO parton fragmentation functions with inclusive charged-particle spectra at hadron colliders, Nucl. Phys. B 883 (2014) 615 [arXiv:1311.1415] [INSPIRE].

[187] Y. Mehtar-Tani and K. Tywoniuk, Jet (de)coherence in Pb-Pb collisions at the LHC, Phys. Lett. B 744 (2015) 284 [arXiv: 1401.8293] [INSPIRE]. 